\title{
Relationships among coleopteran suborders and major endoneopteran lineages: Evidence from hind wing characters
}

\author{
JARMILA KUKALOVÁ-PECK ${ }^{1}$ and JOHN F. LAWRENCE ${ }^{2,3}$ \\ ${ }^{1}$ Earth Sciences, Carleton University, Ottawa, ON K1S 5B6, Canada; e-mail: JarmilaPeck@pigeon.carleton.ca \\ ${ }^{2}$ CSIRO Entomology, GPO Box 1700, Canberra, ACT 2601, Australia \\ ${ }^{3} 130$ Hartwig Road, Gympie, QLD 4570, Australia; e-mail: beetle@spiderweb.com.au
}

Key words. Coleoptera suborders, Endopterygota, Neoptera, Endoneoptera lineages, insect wings, phylogeny, morphology

\begin{abstract}
A phylogenetic analysis of the four coleopteran suborders (Polyphaga, Archostemata, Myxophaga and Adephaga), four other endoneopteran taxa (Strepsiptera, Neuropterida, Mecopterida and Hymenoptera) and three neopteran outgroups (Orthoneoptera, Blattoneoptera and Hemineoptera) is performed based on 63 characters of hind wing venation, articulation and folding patterns, with character states coded for the groundplan of each taxon (not for exemplar genera or species). The shortest tree found using Winclada with Nona exhibits the following topology: Orthoneoptera + (Blattoneoptera + (Hemineoptera + Endoneoptera: $($ Hymenoptera $+(($ Neuropterida + Mecopterida $)+($ Coleoptera + Strepsiptera $)))))$. Homologization of the hind wing venation in Coleoptera is reviewed and updated, and comments are made concerning recent works on wing folding. Recent phylogenetic schemes proposed for the orders of Endoneoptera and suborders of Coleoptera are reviewed and their supporting evidence critically examined. The special role and influence of the hind wing anojugal lobe on the diversification of Neoptera and Endoneoptera is discussed. A scenario is proposed for the origin and evolution of the insect hind wing.
\end{abstract}

\section{CONTENTS}

Introduction and methodology $\ldots \ldots \ldots \ldots \ldots \ldots \ldots . \ldots 6$

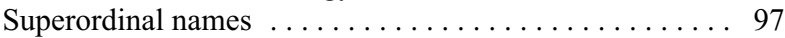

Taxon sampling $\ldots \ldots \ldots \ldots \ldots \ldots \ldots \ldots \ldots \ldots$

Specimen preparation and examination $\ldots \ldots \ldots \ldots .97$

Problems of interpretation and homologization ......998

Terms, abbreviations, graphic symbols and color-coding 98

Review of terms and concepts used by Kukalová-Peck \&

Lawrence (1993) with subsequent changes based on new

homologizations and recent studies $\ldots \ldots \ldots \ldots \ldots \ldots 100$

Definitions ......................... 100

Articular region $\ldots \ldots \ldots \ldots \ldots \ldots \ldots \ldots . \ldots \ldots$

Anterior and posterior wing processes (AWP and PWP) 100

Costal proxalare (PRC) and tegula $\ldots \ldots \ldots \ldots \ldots 100$

Median plate $(\mathrm{FM}+\mathrm{FCu}) \ldots \ldots \ldots \ldots \ldots \ldots \ldots$

Anterior anal basivenale (BAA) . . . . . . . . . 101

Third axillary sclerite $(3 \mathrm{Ax}) \ldots \ldots \ldots \ldots \ldots \ldots 10 \ldots \ldots$

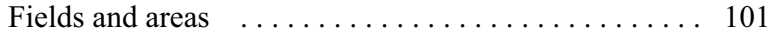

Medial bridge $\ldots \ldots \ldots \ldots \ldots \ldots \ldots \ldots \ldots \ldots \ldots \ldots$

Radial and medial bars .................. 101

Radial and medial loops ................... 101

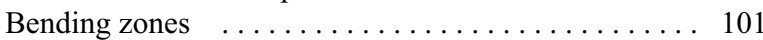

Radial bending zone $\ldots \ldots \ldots \ldots \ldots \ldots \ldots \ldots \ldots$

Medial bending zone $\ldots \ldots \ldots \ldots \ldots \ldots \ldots \ldots \ldots 10 \ldots$

Hinges .......................... 102

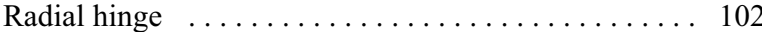

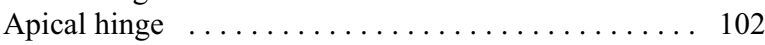

Medial hinge $\ldots \ldots \ldots \ldots \ldots \ldots \ldots \ldots \ldots \ldots . \ldots \ldots$

Radial cell ......................... 102

Apical cell ....................... 102

Wedge cell ........................ 102

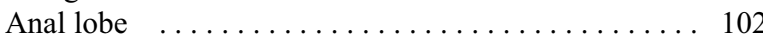

Wing folding and unfolding $\ldots \ldots \ldots \ldots \ldots \ldots . \ldots 2$

Veinal homologies $\ldots \ldots \ldots \ldots \ldots \ldots \ldots \ldots \ldots \ldots$

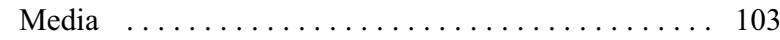

Cubitus ....................... 103
Anal anterior $\ldots \ldots \ldots \ldots \ldots \ldots \ldots \ldots \ldots \ldots . \ldots \ldots$ Specific changes to figures in Kukalová-Peck \& Lawrence (1993) ............................... 104

Origin and diversification of the neopteran wing $\ldots \ldots \ldots 104$

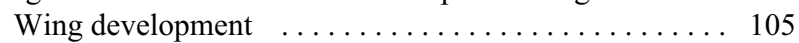

Origin of wing venation $\ldots \ldots \ldots \ldots \ldots \ldots \ldots \ldots \ldots$

Origin of wing articulation $\ldots \ldots \ldots \ldots \ldots \ldots \ldots$

Wing shifting and the origin of the basalare, subalare and

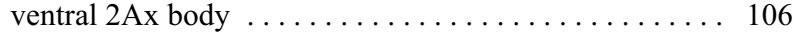

Wing homologues in other Arthropoda $\ldots . . . \ldots . . .106$

Wings in the apterygotes $\ldots \ldots \ldots \ldots \ldots \ldots \ldots \ldots 106$

Neoptera: basal divergence in wing structure ...... 106

Phylogenetic analysis of the major groups of Endoneoptera,

Coleoptera and the suborders of Coleoptera with three basal

neopteran outgroups ........................ 109

Included taxa $\ldots \ldots \ldots \ldots \ldots \ldots \ldots \ldots \ldots \ldots \ldots \ldots$

Orthoneoptera ........................ 109

Blattoneoptera ......................... 109

Hemineoptera ......................... 109

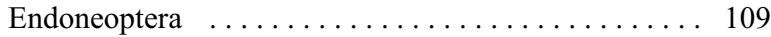

Hymenoptera $\ldots \ldots \ldots \ldots \ldots \ldots \ldots \ldots \ldots \ldots . \ldots \ldots$

Neuropterida ........................... 110

Mecopterida .................... 110

Coleopterida $\ldots \ldots \ldots \ldots \ldots \ldots \ldots \ldots \ldots \ldots \ldots \ldots \ldots$

Strepsiptera . . . ................... 111

Coleoptera ......................... 111

Characters and character state distribution $\ldots \ldots \ldots .112$

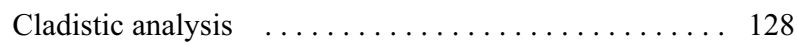

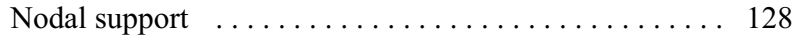

Results and implications ... . . . . . . . . . . . . . . 129

Discussion and conclusion $\ldots \ldots \ldots \ldots \ldots \ldots \ldots \ldots . \ldots \ldots$

Endoneopteran lineages $\ldots \ldots \ldots \ldots \ldots \ldots \ldots \ldots$

Coleopteran suborders $\ldots \ldots \ldots \ldots \ldots \ldots \ldots \ldots \ldots 131$

Phylogenetic relations proposed by Beutel \& Haas (1998,

2000) .......................... 131

Phylogenetic relations proposed and documented here 133

Coleopteran suborders in fossil record ......... 133 
A taxon of doubtful subordinal placement $\ldots \ldots \ldots .134$

Molecular phylogenetic evidence ............. 134

Thorax: structural integrity and phylogenetics . . . . 134

Acknowledgments ........................ 134

References ...................... 135

Appendix 1. Partial Lists of Genera examined ........ 137

Appendix 2. Kukalová-Peck \& Lawrence (1993): Changes in

figure labels $\ldots \ldots \ldots \ldots \ldots \ldots \ldots \ldots \ldots \ldots . \ldots \ldots$

\section{INTRODUCTION AND METHODOLOGY}

In 1993 we proposed a phylogenetic scheme of relationships for the coleopteran suborders based on representatives of 179 genera in 108 families (51 Figs were published). We proposed a homologized veinal nomenclature, and found synapomorphies supporting the following cladogram: (Polyphaga + (Archostemata + (Adephaga + Myxophaga))). This scheme differed considerably from the generally accepted one proposed by Crowson (1960) and first expressed as a cladogram by Klausnitzer (1975): (Archostemata + (Adephaga + (Myxophaga + Polyphaga))). Our results were subsequently challenged by Beutel \& Haas (2000), who found the Crowson scheme to be supported by a large set of characters from several complexes, including thoracic exoskeleton and musculature, as well as wing venation and folding. This challenge is to be applauded for its attempt to bring a large suite of characters to bear on an important problem in phylogenetics and to apply modern cladistic techniques for the first time at the subordinal level in beetles. It has also stimulated us to reexamine the wing characters used in our previous paper (KukalováPeck \& Lawrence, 1993), revise some previous homologizations, expand the hind wing character set, and provide a cladistic analysis of beetle suborders and major lineages of Endoneoptera (Endopterygota, Holometabola) with three basal neopteran outgroups. In the last decade, one of us (JKP) researched the groundplans of the Neoptera superordinal lineages (see character table in Haas \& Kukalová-Peck, 2001). The resulting improved homologization of anojugal lobes in the hind wings also affects the Coleoptera. We use this opportunity to update and simplify our previous nomenclature, and to also correct veinal interpretation in some of our figures. Due to the notorious but inevitable snowballing effect ruling higher phylogenetics, we ultimately found it necessary to emend the veinal systems of the other Endoneopteran orders. As expected, a new phylogenetic scheme of Endoneoptera resulted after using the homologized veinal system, which is tabulated below.

The morphological characters usually used to evaluate the relationships of the higher taxa of Insecta are plagued by homoplasies, which are so prevalent and convincing that they overwhelm the system (Kristensen, 1975, 1981, 1991, 1995, 1997, 1999). The morphological characters of the insect orders used in recent phylogenetic analyses by Beutel \& Gorb (2001) or Wheeler et al. (2001) are very difficult to assess because the Paleozoic or early Mesozoic groundplan character states, indicative of the inter-ordinal relationship, have been thoroughly obscured by later transformations. Thus, many higher-level "syna- pomorphies" are uncertain and likely to be undetected homoplasies. This is almost never a problem when old, large, richly diversified and (above all else) correctly and fully homologized morphological complexes are compared in detail for a wide range of taxa representing different orders, suborders and superfamilies (Haas \& Kukalová-Peck, 2001). These complexes are composed of many subordinated and integrated characters, which can be searched for differences simultaneously in all higher taxa involved in a study. If we can also ascertain that changes in character states follow a certain set of rules, then the separation of synapomorphies from homoplasies is facilitated.

The wings of pterygote insects provide an ideal character complex for such a study for a number of reasons.

(1) Most insect wings are very easy to examine with a minimum of preparation. They are only slightly less accessible in Coleoptera, where they are folded beneath the elytra. Since a larger number of taxa may be examined in a given time period, it is possible not only to better demonstrate the range of in-group variation but to discover primitive groundplan attributes retained in only a small portion of extant taxa.

(2) Insect wing venation is essentially two-dimensional, making character states easier to observe and record. It must be noted, however, that veinal fluting, resulting in the alternating convexity and concavity of paired veins, or modifications in the wing struts associated with flight or folding require three-dimensional observation (see below).

(3) Hind wing characters in most insect groups are easily observable in fossil specimens. Although this does not apply to Coleoptera, where an unfolded or partly folded hind wing is rarely preserved (see Kukalová-Peck \& Lawrence 1993, p. 186), wings of other endoneopteran insects and their ancestors among the more basal neopteran orders are relatively frequent in the fossil record.

(4) The flight function of the hind wing is obvious, and aerodynamic features of the wing have been studied in a number of groups. This may be contrasted with the male and female reproductive organs (Büning, 1998; Büning \& Maddison, 1998), where the details of sperm storage and transfer, egg formation and oviposition may be obscure. The folding and unfolding process also affects hind wing structure and venation, particularly in Coleoptera; but recent studies of folding mechanics have shed some light on this (Beutel \& Haas, 2000; here on p. 102).

Both neontologists and paleontologists working with insect wing venation have found that certain sequences of character state changes appear to be irreversible. This may be expressed as two rules which have been abundantly used and tested by systematists for more than a century (Carpenter, 1992; Haas \& Kukalová-Peck, 2001; Hennig, 1969, 1981; Kukalová-Peck, 1983): (1) all veinal reductions and fusions into stems and veinal braces are derived, and (2) veinal fusions near the base never unfuse and lost veins never reappear. The second of these rules requires some clarification. The 
derived fusion of the pairs of veinal sectors RA-RP, MA-MP and CuA-CuP into the derived veinal stem of $\mathrm{R}$, $\mathrm{M}$ and $\mathrm{Cu}$, and the derived long fusion between two principal branches (such as MA $+\mathrm{R}+\mathrm{RP}$ in Blattoneoptera, Hemineoptera and Endoneoptera, $\mathrm{CuA}+\mathrm{M}+\mathrm{MP}$ in Orthoneoptera and MP + RP + MA in Hymenoptera) are irreversible, but there is variation in many cross-vein braces (e.g., mp-cua and rp-ma), which can be expressed as either cross-veins or as short fusions, or obscured by other wing features (mp-cua is destroyed by a fold in most Coleoptera). The fused branches RP + MA may separate again apically (forming what appears to be a "new" apical branch, a plesiomorphy), or stay fused together until the wing margin (an apomorphy).

Character states in higher taxa are phylogenetically informative only at their groundplan level (Hennig, 1981). Therefore, a phylogenetic analysis is realistic only when based on attributes considered to be part of the groundplan. We used the term groundplan to mean the set of most plesiomorphic character states available in a very broad sample of representatives of a higher taxon. When constructing venational groundplans, we chose those character states exhibiting the fewest fusions, reductions or other alterations, with respect to the pterygote (protowing) and neopteran groundplans (Fig. 1A, B, C). Those for orders and suborders were based on the study of living representatives (Appendix 1), while those for superordinal lineages were based on living and fossil representatives. For further documentation see Haas \& Kukalová-Peck (2001).

The flight-adapted groundplan combination of veinal stems, forks, fusions, braces and reductions are the phylogenetically informative attributes, which "bind" each pterygote higher taxon together and make it visually recognizable even in highly derived species. But in spite of their crucial significance and deep influence, higher groundplan characters are usually obscured by later changes and often "invisible" in the majority of recent species. They typically survive only in a few representatives scattered among several families. A larger, more complete sample and a broader study invariably yield more reliable groundplans. Color figures of the coleopteran subordinal groundplans are given in Figs 19-22 for the distal portion of the wing (radial, central and apical fields) and Figs 23-26 for the proximal portion (medial and anal fields). These groundplans are falsifiable when more plesiomorphic states, unknown to us, are discovered in the future.

Many workers object to the groundplan method as one in which a subjective decision is made prior to the cladistic analysis, and thus base all their analyses, including those of higher taxa, on exemplar genera or species. One of us (JFL) who normally uses exemplars and has discussed their use with colleagues, has observed again and again how much time is spent selecting exemplars for higher taxa which display the greatest number of plesiomorphic features for that taxon. Some workers choose exemplars with the most commonly observed character states for that taxon, the rationale being that those which are most widely distributed taxonomically must be plesiomorphic, or that "common equals primitive", while others code anything that is on hand or easy to obtain, the rationale being that if enough characters are used, resulting homoplasies will "somehow" be swamped by true synapomorphies. In reality the reverse is usually true.

\section{Supraordinal names}

Names of supraordinal taxa used throughout this paper are based on generally known groups, as proposed by Kukalová-Peck \& Brauckmann (1990) and used by Haas \& Kukalová-Peck (2001) for major taxa of Neoptera (subdivisions of Kristensen 1991) and those used by Kristensen (1991) and Kukalová-Peck (1991) for the sections of Endoneoptera (= Endopterygota or Holometabola). The ordinal components of these higher taxa are listed below. Martynov (1923) divided Neoptera into Polyneoptera (pleconeopterans + orthoneopterans + blattoneopterans), Paraneoptera (hemineopterans), and Oligoneoptera (endoneopterans). The wing characters, however, indicate that Polyneoptera is a paraphyletic group, the name for Paraneoptera (if the order Zoraptera is excluded: Haas \& Kukalová-Peck, 2001, p. 486) should be Acercaria, and Oligoneoptera are not frequently used or generally familiar. The name Endoneoptera has been favoured over the commonly used Endopterygota or Holometabola to keep the names of higher neopteran taxa formally consistent.

\section{Taxon sampling}

A large number of beetle wings were studied in preparation for our 1993 paper and many more dissections were made by JFL since that time in connection with other projects, including the world interactive key to adult beetles (Lawrence et al., 1999). The wing venation and articulation of a wide variety of pterygote insects were examined by JKP in connection with several parallel studies, including that recently published by Haas \& Kukalová-Peck (2001). Appendix 1 includes most of the beetle genera examined, plus a selection of taxa from other orders. Family and superfamily concepts for Coleoptera are from Lawrence \& Newton (1995), Lawrence et al. (1999) and a few more recent works (Lawrence, 2001; Leschen, 2003). Larger samples of curculionoid wings are illustrated in the survey by Zherikhin \& Gratshev (1995), but note that a different veinal nomenclature was used in that paper.

\section{Specimen preparation and examination}

As indicated in our previous paper, although wings were prepared in various ways, the type of wing preparation found to be necessary for examining venation and articulation, as well as folds, is the dry mount. For the best results, dry beetle specimens were placed in potassium hydroxide for a brief period only, and then placed in alcohol, while the hind wings were removed. In removing the wing from body, an attempt was often made to leave axillary sclerites attached, but this was not always possible. The wing was then removed from the alcohol, allowed to dry out slightly and then placed onto a drop of water on a microslide. Sometimes a wing was allowed to remain in the folded position, but usually it was gently 
manipulated, so that it completely unfolded onto the surface of the water bubble, and then allowed to dry onto the slide (further manipulation was sometimes necessary during the drying process). In general, both wings were mounted facing to the right, so that the right wing was dorsal side up and the left one ventral side up. When the two wings were dry, four small drops of glue were placed on the slide and then a square cover slip was placed over them. This protected the wings while allowing an air interface to remain between them and the coverslip. Occasionally a folded wing was mounted in the same fashion, or wings were mounted while still attached to the metanotum. Large preparations could not be covered, and sometimes a drop of glue was used to keep them on the slide. Usually, the remainder of the beetle specimen was then properly macerated for the study of other structures. If the wings are not removed before maceration, they may be used to trace major veins, but folds and other features associated with folding may be difficult or impossible to observe properly. In extreme cases, the two cuticular layers may separate, making it difficult even to follow the veins.

Special preparations were sometimes necessary in order to properly examine the axillary region. The recently killed or partly cleared and softened beetle specimen was pinned and the wing spread out over a foam ridge and then pulled down and attached at a lower level than the body, so that the entire axillary region was exposed. The preparation was then allowed to dry out. This was found to be greatly preferable to wet preparations, because most weakly pigmented wing veins, sclerites, ligaments and sutures are invisible in a liquid medium.

\section{Problems of interpretation and homologization}

Although veinal reductions and fusions in the groundplans continue from the family to genus and species level, these more recent changes are less stabilized. As a rule, veins in lower taxa are strongly influenced by the size and shape of elytra, and by the length of hind wings. Veins may be crossed by a large number of folds, which sometime form patterns typical for certain families or tribes. Short folds quite often "erase" parts of the veins and remaining veins may be rearranged in new, unusual patterns, which mimic the arrangement of principal veins (especially in the medial field). One of the best examples of this concerns the cross-vein r3 in Polyphaga, which loses its connection to RP and in elongate beetles may become longitudinally oriented, thus mimicking a major vein. This is the so-called radial recurrent in the Forbes (1922) system of veinal nomenclature (Kukalová-Peck \& Lawrence, 1993).

In some areas of the wing membrane having limited venation, secondary sclerotizations may develop which are sometimes vein-like; these are usually very different from the original venation and unlikely to be confused with it. Examples are the apical "ghost branches" in Sphaerites and Trox illustrated in Kukalová-Peck \& Lawrence (1993, Figs 41, 50). Somewhat more difficult to interpret are the apical sclerotizations occurring in various Elateroidea, which usually consist of one or two oblique struts or sometimes three struts forming a sigma; it is not certain if they are secondary sclerotizations or reduced and distorted apical remnants of RA and RP. The most problematical features are usually encountered when working with relatively small samples, which is often the case with highly diversified and very broadly distributed taxa, and with rare taxa (prohibitive factors being time and material); they are genetic or developmental anomalies, which are not really separable from other veins. Included are apical forks, usually close to the wing margin, and less commonly subdivisions of the radial or wedge cell in Polyphaga. In most cases these occur on one wing only of a single individual, but they may be present in both wings but not repeatable in other wings of the same species. A few of these were illustrated in Kukalová-Peck \& Lawrence (1993) and are discussed in Appendix 2. As in other Pterygota some reduced venation, especially in small-sized beetles, is very difficult or impossible to fully homologize.

\section{Terms, abbreviations, graphic symbols and color-coding}

Alula. Basal subdivision of the anal lobe in the fore wing of Diptera.

Anal fold. Fold running between anal anterior vein AA and anal posterior vein AP.

Archipleuron. All arthropod limb-derived appendages are serially homologous and share a single 11-segmented groundplan mode. The first podite, epicoxa, flattened into a plate called archipleuron, which was articulated to the tergum, and which served as an articular site for 10-segmented cylindrical podites, all articulating with subcoxa. In Insecta, the archipleuron survived only in the thorax: in Archaeognatha and fossil Monura, as an entire pleural plate and in Pterygota, as a plate fragmented into pteralia; elsewhere, it became fully fused with the tergum (Kukalová-Peck, 1997).

Arculus. Alternative term for mp-cua brace present in all Endoneoptera + Hemineoptera and in some Blattoneoptera.

AX. Axalare (axalaria).

1, 2, 3, 4Ax. First, second, third, fourth axillary sclerite; composite, irregular sclerites occurring only in Neoptera.

1Ax arm. Radial axalare, subcostal axalare, subcostal fulcalare $(\mathrm{AXR}+\mathrm{AXSc}+\mathrm{FSc})$ fused together.

1 Ax body. Medial proxalare PRM.

$2 \mathrm{Ax}$ body and arm. Medial axalare AXM and radial fulcalare FR.

3Ax goblet \& heel. Cubital axalare $\mathrm{AXCu}$ subdivided by a fold into larger, protruding goblet and smaller, more or less receding heel; goblet is articulated with saucer, heel with cubital arm.

3Ax saucer. Anojugal axalare AXAJ (AXA + AXJ fused). Saucer is articulated with $3 \mathrm{Ax}$ goblet, cubital arm, anal arm and jugal arm, and with 4Ax (or PWP).

3Ax cubital, anal, jugal arm. Cubital fulcalare $\mathrm{FCu}$, anal fulcalare FA, jugal fulcalare FJ.

3Ax fragment. Fragment of $3 \mathrm{Ax}$ goblet bearing insertion of wing flexor muscle.

3Ax plate. $3 \mathrm{Ax}$ goblet $\&$ heel + saucer + three arms $(\mathrm{AXCu}+$ $\mathrm{AXAJ}+\mathrm{FCu}+\mathrm{FA}+\mathrm{FJ})$ fused; $3 \mathrm{Ax}$ plate occurs only in Coleoptera and Strepsiptera.

4Ax. Anal + jugal proxalare (PRA + PRJ) fused, articulated to the tergum; when secondarily fused with the tergum, 4Ax is called posterior wing process PWP. 
AA, AP. Anal anterior, anal posterior; anterior and posterior anal vein.

AWP. Anterior wing process, subcostal + radial proxalare (PRSc + PRR) fused together.

AXA, AXAJ. Anal axalare, anojugal axalare (AXA, AXJ) fused together form AXAJ (=3Ax saucer).

B. Basivenale (basivenalia), sclerotized veinal blood sinus at the wing base.

BA, BAA, BAP. Anal basivenale, anal anterior basivenale, anal posterior basivenale.

BAS. Basalare (ventral pteralium).

BCu, BJ, BM, BR, BSc. Cubital basivenale, jugal basivenale, medial basivenale, radial basivenale, subcostal basivenale; each basivenale gives rise to two veinal sectors.

BMCu. Medial + cubital basivenale, fused.

C, CA, CP. Costa, costa anterior, costa posteior; symbol $\mathrm{C}$ is used below for precostal strip + costa, fused together into the anterior margin; $\mathrm{CP}$ is present in many fossils but reduced in most modern Neoptera.

Claval line (fold). Flexion line originally running between cubitus posterior $\mathrm{CuP}$ and anal anterior $\mathrm{AA}$.

Cross-vein brace. Important cross-vein between two principal veins.

$\mathrm{Cu}, \mathrm{CuA}, \mathrm{CuP}$. Cubitus (the stem of cubitus), cubitus anterior, cubitus posterior.

$\mathbf{C u A}+\mathbf{M}+\mathbf{M P}$. CuA fuses basally to M, then to MP, and separates apically from MP; this long veinal brace is typical for Orthoneoptera.

F. Fulcalare (fulcalaria).

FA, FCu, FJ, FM, FR. Anal fulcalare (3Ax anal arm); cubital fulcalare (3Ax cubital arm), may become abutted to median plate FM; jugal fulcalare (3Ax jugal arm); medial fulcalare (median plate); radial fulcalare (2Ax arm).

Full anojugal lobe. Anojugal lobe composed of AA, AP, JA, JP veins; lobe starts at the claval flexion line.

HP. Humeral plate, costal fulcalare + costal basivenale $(\mathrm{FC}+$ $\mathrm{BC}$ ) fused together and with precostal strip; sutures indicative of fusions are very rarely preserved.

Humeral vein. Conspicuous convex cross-vein brace in costal area not far from base.

J, JA, JP. Jugal, jugal anterior, jugal posterior.

Jugal fold. Convex fold running between anal posterior AP and jugal anterior JA.

M, MA, MP. Media (the stem of media), media anterior, media posterior (veinal sectors which fuse secondarily into the stem of M).

Median plate, medial plate. Medial fulcalare FM; sometimes fused with cubital fulcalare $\mathrm{FCu}$ (3Ax cubital arm) into an extended median plate; median plate is subdivided only in Coleoptera.

m-cua. Medio-cubital cross-vein brace found in modern Pleconeoptera $(=$ Plecoptera + Embioptera $)$, but absent in their fossil stem group.

mp-cua. Posteromedio-cubital brace (cross-vein or short fusion) also known as arculus, found in some Blattoneoptera and all Hemineoptera + Endoneoptera, but absent in Pleconeoptera and Orthoneoptera

Medio-cubital line. Flexion line between MP and $\mathrm{CuA}$, prominent in the hind wings of Neuropterida.

mp1+2-mp3, mp1+2-mp3+4, mp3+4-cua. Prominent crossvein braces in Coleoptera.

MP4 + CuA1 (MP4 + CuA1+2, MP3+4 + CuA1+2). Groundplan veinal fusion between utmost posterior MP branch and utmost anterior $\mathrm{CuA}$ branch, found in all Endoneoptera lineages.
Nygmata (sing. nygma). Spots on the wings of Neuropterida, Mecoptera, Trichoptera and Hymenoptera: Symphyta, probably sensory organs. Presumed lost in Coleopterida, Diptera and Lepidoptera.

Partial anojugal lobe. Diminished lobe composed of AP, JA, JP veins; it starts at the anal fold.

PC, PC strip. In Pterygota, precosta is transformed into precostal strip; in Neoptera, it is fully fused anteriorly with costal proxalare PRC, costal axalare AXC, humeral plate $\mathrm{HP}$ and costal margin

PR. Proxalare (proxalaria).

PRA, PRAJ, PRC, PRCu, PRJ, PRM, PRR, PRSc. Anal proxalare, anojugal proxalare, costal proxalare, cubital proxalare, jugal proxalare, medial proxalare, radialproxalare, subcostal proxalare.

PWP. Posterior wing process (PRA + PRJ) fused with the tergum; homologue of 4Ax, derived.

R, RA, RP. Radius (the stem of radius), radius anterior, radius posterior.

ra-rpma bridge. Broad brace of thickened membrane connecting basally RA and RP + MA in Strepsiptera (instead of the stem of radius).

radio-medial line. Flexion line, usually running anteriorly of media.

ra1, ra2, ra3, ra4. Prominent cross-vein braces between $\mathrm{RA} 1+2$ and $\mathrm{RA} 3+4$ in Coleoptera.

r3, r4. Prominent cross-vein braces between RA3+4 and RP in Coleoptera.

rp-mp1, rp-mp2. Prominent cross-vein braces between RP and MP1+2 in Coleoptera.

rpma-mp (rma-mp). Important endoneopteran brace between $\mathrm{RP}+\mathrm{MA}$ and MP (or R + MA and MP), expressed in Hymenoptera as a long fusion at mid-wing, and in Coleoptera + Neuropterida + Mecopterida, as a long cross-vein near base: sinusoid cross-vein in Neuropterida, medial bridge in Coleoptera, strong bar in Mecopterida: Diptera; obscured by abutment of R + MA and MP in Mecoptera, Trichoptera, and Lepidoptera.

Sc, ScA, ScP. Subcosta, subcosta anterior, subcosta posterior; ScA was present in many fossil Neoptera and in Paleoptera, but became reduced in most modern Neoptera.

ScA bulge. Bulging ScA area occurring only in Endoneoptera.

Stem (veinal stem). Sectors of the three central veins, radius, media, cubitus (RA-RP, MA-MP, CuA-CuP) are either separate (a plesiomorphy) or they often fuse basally into a veinal stem (an apomorphy). As a higher character, veinal stem never reverses to sectors, and it often becomes longer in the derived taxa.

SUB. Subalare.

TEG. Tegula.

Veinal brace. Two principal veins fuse for a short distance, then separate again.

Veinal sector. Except for precosta (PC) forming precostal strip, each wing vein is composed of two sectors, anterior $\mathrm{A}$ and posterior P: CA, CP; ScA, ScP; RA, RP; MA, MP; CuA, CuP; AA, AP; JA, JP; only central sectors form veinal stems (of R, $\mathrm{M}, \mathrm{Cu})$.

VWP. Ventral wing process of the subcoxal pleuron supporting ventrally the wing, usually placed under $2 \mathrm{Ax}$ body.

Color code for morphological features

Orange. Precostal strip $+\operatorname{costa}(\mathrm{PC}+\mathrm{C})$ fused, costal proxalare PRC, tegula TEG, humeral plate HP; jugal veins J, JA, JP, jugal axalare AXJ, 3Ax jugal arm FJ. When fused into AXAJ in $3 \mathrm{Ax}$ saucer, AXJ is orange and AXA yellow. 
Yellow. Subcosta Sc, subcostal proxalare PRSc (part of the anterior wing process AWP), and AXSc + FSc (fused together in the 1Ax arm); anal A, anal proxalare PRA (part of the posterior wing process PWP), AXA (part of $3 \mathrm{Ax}$ saucer) and FA (3Ax anal arm).

Red. Media M, 1Ax body (medial proxalare PRM), 2Ax body (medial axalare AXM), median plate (medial fulcalare FM).

Green. Cubitus $\mathrm{Cu}$, cubital proxalare $\mathrm{PRCu}$ (dotted when desclerotized), 3Ax goblet and heel (cubital axalare $\mathrm{AXCu}$ ), cubital fulcalare $\mathrm{FCu}$ (separate $=3 \mathrm{Ax}$ cubital arm; often joins FM to form an extended median plate).

Blue. Radius R, radial proxalare PRR (part of the anterior wing process AWP), radial axalare AXR (base of $1 \mathrm{Ax}$ arm), radial fulcalare FR (2Ax arm).

Muddy yellow. 3Ax anal arm in Fig. 35.

Light blue. 3Ax cubital arm FCu in Fig. 35.

Brown. Subalare (SUB) and basalare (BAS).

\section{REVIEW OF TERMS AND CONCEPTS USED BY KUKALOVÁ-PECK AND LAWRENCE (1993) WITH SUBSEQUENT CHANGES BASED ON NEW HOMOLOGIZATIONS AND RECENT STUDIES}

\section{Definitions}

Most of the terms discussed below were used in our 1993 paper, either for the first time or following earlier workers like Schneider (1978). The most important of these are repeated here and in some cases emended, due not only to their importance in wing support or in the folding-unfolding process, but to the somewhat inconsistent use of the terms both in the 1993 paper and in subsequent works dealing with beetle wings (Beutel \& Haas, 2000; Lawrence et al., 1999). Some terms, which are adequately described by Kukalová-Peck \& Lawrence (1993) or Haas \& Kukalová-Peck (2001), are included below because of their misapplication in recent publications.

Articular region. The region of articulation between the wing and the tergum is highly complex and provides an excellent source of phylogenetically useful charactes; unfortunately, its complexity has also led to a number of errors in the interpretation of individual axillary sclerites and homologization of these sclerites across the insect orders. As in venational studies, the use of axillary characters to assess relationships of higher taxa requires the same full homologization of all articular sclerites at the Pterygota and Neoptera level and a similar construction of ordinal and subordinal groundplans. Although a number of articular regions were examined by us in connection with our previous paper (Kukalová-Peck \& Lawrence, 1993, Figs 72-81), little use was made of axillary characters in our study of coleopteran suborders. However, a detailed homologization of axillary elements in the basal neopteran orders was offered by Haas \& KukalováPeck (2001) and a further study of this region in Endoneoptera, including Coleoptera, has been recently carried out by one of us (JKP).

In the most recent work on wing articulation in Endoneoptera, Hörnschemeyer (1998, 2002) provided some useful and previously unknown morphological observations. However, when evaluating these axillary characters, he arrived at some "astonishingly different" phylogenetic conclusions from those presented in the papers cited above. This was due mainly to incorrect homologization and reversed polarities, as pointed out below. The neopteran groundplan used by Hörnschemeyer as the reference scheme for wing base homologization and nomenclature (1998, Fig. 1; 2002, Fig. 1A) is from a Snodgrass (1935) figure based mainly on Acrididae (Orthoptera). According to this scheme, the region contains three axillary sclerites (conceived as "prime" sclerites rather than composite ones), two median plates (proximal and "distal"), the tegula, the humeral plate, and three "tergal" projections called notal wing processes, anterior (ANP), median (MNP), and posterior (PNP). This scheme contains autapomorphic features occurring only in Orthoptera, and lacks numerous basal neopteran sclerites, which are present in the fully homologized pterygote and neopteran reference schemes offered by Kukalová-Peck (1983, Fig. 15; 1997, Fig. 19.8) and Haas \& Kukalová-Peck (2001, Figs 1A-C).

Anterior and posterior wing processes (AWP and PWP). These two features were considered by Snodgrass (1935) to be projections of the tergum; thus Snodgrass and most other authors following him have used the terms anterior and posterior notal processes (ANP and PNP). Since Stenzhorn (1974) has demonstrated in an experiment with larval transplants that these processes separate from wing Anlagen and are not tergal derivatives, we feel that the more neutral term "wing process" is preferable. When homologized, the anterior wing process is composed of two sclerites, the subcostal proxalare (PRSc) and radial proxalare (PRR), which are often fused but separated by a suture or sometimes an additional gap (in Plecoptera). Primitively, the sclerites are separated from and articulated with the tergum (as in Megaloptera and some Mecopterida) and the fusion with the tergum is a derived condition. Similarly, the posterior wing process is formed by the anal and jugal proxalaria (PRA and PRJ) separated by a suture or a gap and articulated with the tergum. This plesiomorphic composite sclerite, which occurs in many Plecoptera, Orthoptera, Hymenoptera, Neuropterida and Mecopterida, is often referred to as the fourth axillary sclerite $(4 A x)$. The derived process, fused with the tergum, formed repeatedly in these groups and occurs in Trichoptera, Lepidoptera and most Coleoptera.

Costal proxalare (PRC) and tegula. Costal proxalare (PRC) is present in both Strepsiptera and Coleoptera but the tegula (an elevated cluster of sensory setae occupying the position of the costal axillare (AXC) in the basal neopteran wing), is absent (Figs 1, 29, 30). In Strepsiptera Hörnschemeyer (1998) erroneously interpreted the enlarged PRC as a "tegula", although it was atypically close to the tergum. In Coleoptera Hörnschemeyer recognized and figured the costal proxalare (PRC) as different from a tegula in the archostematan beetle Priacma, but called it a "prealar sclerite" (following Baehr, 1975).

Median plate (FM + FCu). The median plate (also called medial plate) in most insects is a sclerite formed by the medial fulcalare, which is sometimes combined with the cubital fulcalare, called the cubital arm of $3 \mathrm{Ax}$. In the 
plesiomorphic condition the two are separate, but in a derived state they may be fused (as in most Plecoptera) or membranized (as in Dermaptera). In Hemineoptera and Endoneoptera, FM and FCu are separate, but in Strepsiptera and Coleoptera $\mathrm{FCu}$ is fused with the $3 \mathrm{Ax}$ plate (Fig. $35 \mathrm{~L}, \mathrm{M}$ ), so that $3 \mathrm{Ax}$ articulates directly with $\mathrm{BCu}$ (Fig. 31) (a complex synapomorphy). In the axillary scheme of Snodgrass (1935), there is a "proximal" and "distal" median plate, separated by a wing-flexing fold. The "proximal" median plate is on the "body" side of the articulation and is homologous to the separate FM and $\mathrm{FCu}$, but the "distal" plate occurring on the "wing" side of the articulation, is formed by the basivenalia BM + $\mathrm{BCu}$ delimited by secondary folds, and is present only in Orthoptera (in grasshoppers and some tettigoniids). In Coleoptera, the median plate is formed entirely of FM and uniquely subdivided. Hörnschemeyer (1998) incorrectly interpreted this subdivided FM as the proximal and distal median plates of Snodgrass, an impossibility considering that the coleopteran subdivisions both lie on the same "body" side of the articulation as the axillary sclerites and other pteralia.

Anterior anal basivenale (BAA). In our 1993 paper, we mentioned a peculiar V-shaped BAA preserved in some Archostemata and Adephaga (a possible synapomorphy, not observable in Myxophaga). Hörnschemeyer (1998, p. 65) confused this unusual character state of BAA for an ubiquitous presence of BAA in the higher taxa of Neoptera and Pterygota, which is part of our groundplan and was never disputed.

Third axillary sclerite (3Ax) (Fig. 35). Hörnschemeyer $(1998,2002)$ interpreted $3 \mathrm{Ax}$ as a prime sclerite, which in Neuropterida became secondarily fragmented. In the homologized scheme, $3 \mathrm{Ax}$ is composed of five articulated sclerites, $\mathrm{AXCu}$ (folded into a larger protruding goblet and a smaller, flatter heel), AXAJ (saucer) and three arms - cubital (FCu), anal (FA) and jugal (FJ). These pteralia are mostly retained and articulated in Blattoneoptera; in Plecoptera and Orthoneoptera the jugal arm is reduced. In the wings of Endoneoptera, the $3 \mathrm{Ax}$ goblet and saucer sometimes separate. In some Neuropterida hind wings the saucer becomes V-shaped and in Neuropterida and Mecopterida it may be interrupted by one or two folds (Fig. 35O, Q). FA and FJ are fused with the saucer only in Endoneoptera and Hemineoptera, and in Coleoptera and Strepsiptera all five elements of $3 \mathrm{Ax}$ are fused into a single plate (Fig. 35L, M).

Fields and areas. The term "field" refers to a region of wing membrane, which is delimited by veins or folds and used for general descriptive purposes. The fields include humeral, radial, central, apical, medial and anal, but only the last three have proven to be useful for descriptions. The apical field includes everything apical to the radial bar, radial or apical cells, cross-vein $\mathrm{r} 4$, the oblongum cell or medial hook (cross-vein rp-mp2), and the medial spur (apical portion of MP1+2). The medial field includes everything between $\mathrm{MP} 1+2$ and the anal fold and thus includes branches of $\mathrm{MP} 3+4, \mathrm{Cu}$ and $\mathrm{AA} 3+4$. Finally, the anal field includes everything between the anal fold and the wing base, including branches of AP and J. The term "area" is not synonymous with field, but refers to the region occupied by a particular veinal system.

Medial bridge. This refers to the sub-basal brace or clamp which joins the two wing struts (radial and medial bars) and plays a major role in wing unfolding. As indicated below (under Media in Veinal homologies), the bridge appears to be not part of MA but an important cross-vein brace rpma-mp close to base, which occurs also in Neuropterida (Figs 5-9) and Mecopterida (in Diptera, Fig. 33), but in Hymenoptera it forms a long fusion $\mathrm{RP}+\mathrm{MA}+\mathrm{MP}$ at mid-wing (Figs 3-4).

Radial and medial bars (Figs 19-22). These terms refer to the anterior and posterior struts of the beetle hind wing, which are important in wing folding and unfolding, as well as support in flight. The radial bar consists of a long, reinforced section of RA combined with $\mathrm{ScP}$, and it ends well before the wing apex, where it is usually attached to a cell, forming the radial loop. The medial bar consists of the main portion of MP1+2 and ends at the oblongum cell or medial hook, forming the medial loop.

Radial and medial loops (Figs 19-22, 27). The term "loop" was used by Kukalová-Peck \& Lawrence (1993) for the (radial or medial) strut or bar plus an apically attached cell or hook. We have found it more useful to restrict the term "loop" to the apical cell or hook only. Note that the radial loop is formed by the radial cell, which in each suborder is composed of different, nonhomologous veinal elements. The most striking difference in radial cells occurs between Polyphaga and the remaining suborders. The radial loop may or may not enclose a pigmented pterostigma. The medial loop is formed by either the oblongum cell or by the medial hook (the latter is present only in Polyphaga). Each loop is attached to the end of a wing strut (radial or medial bar).

Bending zones (Figs 19-22, 27). A bending zone or deflexion zone (Biegungszone of Schneider, 1978) is a region of reinforced wing vein, usually part of the anterior wing strut (radial bar, $\mathrm{RA}+\mathrm{ScP}$ ) or posterior wing strut (medial bar, MP1+2), which is capable of bending in one direction only when the bar is rotated. This one-way bending seems to be caused in some cases by a series of corrugations or crimps along one surface of the vein or in others by a flattening of the vein. In our 1993 paper these were called springs, but that term is inappropriate, since it implies an intrinsic elasticity, which has not been demonstrated.

Radial bending zone. A bending zone located on the radial bar. In Adephaga and most basal Polyphaga this zone is relatively long, in Ommatidae and some Myxophaga it may be shorter and almost hinge-like, in Cupedidae and Micromalthidae it is replaced by a radial hinge, and in many derived Polyphaga it is lost. This was called the radial spring by Kukalová-Peck \& Lawrence (1993, Figs 98-99).

Medial bending zone (Figs 19-22, 27). A bending zone located on the medial bar. This occurs just proximad of cross-vein rp-mp2 or the medial hook in some basal Polyphaga. Except in some of the larger Bostrichidae, the 
medial bending zone lacks the transverse crimping which often characterizes its radial counterpart, but this portion of the medial bar is instead weakened or flattened.

Hinges. A hinge is any sharp angle or joint in a reinforced wing vein, where a fold intersects that vein. Hinges, as here defined, occur only on the two main wing struts (radial bar or medial bar). In other areas of the wing, intersections of veins and folds tend to be accompanied by a weakening or desclerotization of the vein. In an unfolded wing, the hinge is indicated by a distinct break (membranization) in the strut, an abrupt narrowing or a sharp line. This is synonymous with "joint" as used by Beutel \& Haas (2000) and does not include the folding points (Faltstelle of Schneider, 1978), which occur where a fold reaches the unreinforced wing margin.

Radial hinge. A hinge occurring on the radial bar just before (proximad of) cross-vein ra1 in Cupedidae, Micromalthidae and Myxophaga.

Apical hinge. A hinge occurring just beyond (apicad of) the end of the radial bar in those wings in which sclerotization of the anterior wing margin continues beyond the end of the radial bar. It appears to be derived from the pinch formed by the uniting RA $1+2$ and RA $3+4$ at the distal end of the radial cell, usually followed by the separation of RA3 and RA4 (see Fig. 19). Schneider (1978) referred to this hinge as the Randgelenk (marginal joint), and this has been followed by Beutel \& Haas (2000) and Haas \& Beutel (2001). It is most distinct in Hydrophiloidea and Scarabaeoidea, but in Staphylinoidea, RA has moved away from the wing margin, so that the hinge appears more like a simple fold adjacent to the abrupt end of the radial bar. In most other polyphagans, the apical vein remnants are greatly reduced and the apical hinge completely absent.

Medial hinge (Figs 19-22, 27). A hinge occurring just proximad of the cross-vein rp-mp1 in Archostemata, Adephaga and Myxophaga. In Archostemata, this hinge is less abrupt and may represent a transitional stage between a short bending zone and a true hinge.

Radial cell (Figs 19-22, 27). This term may apply to any cell formed by elements of the radial system, but it is used specifically to refer to a cell in Polyphaga which is formed by the forking and subsequent fusion of RA1+2 and RA3+4, forming an eyelet, from which one or two radial cross-veins (r3 and r4) may emerge. This cell in Polyphaga is not homologous to those cells formed by various radial cross-veins in Adephaga, Myxophaga and Archostemata; however, the cells appear to be analogous in forming the radial loop, which plays a part in wing folding and unfolding.

Apical cell (Figs 19-22, 27). A term introduced here for the most distal of the cells formed between radial cross-veins in some Archostemata and Adephaga. This cell is sometimes referred to as 3R (Hamilton, 1972; Ward, 1979).

Wedge cell (Figs 23-26). This term is commonly used in Coleoptera to refer to the $2^{\text {nd }}$ cubito-anal cell, which is formed by $\mathrm{CuP}, \mathrm{CuA}, \mathrm{CuA} 3+4$ and $\mathrm{AA} 3$ in all basal Coleoptera. It has also been called the anal cell.
Anal lobe (Figs 23-26, 31). This term is often used in Coleoptera to refer to an anojugal lobe which is set off from the remainder of the wing by a distinct anal embayment (either a shallow notch or a deep incision). This lobe is absent in Archostemata and present in Myxophaga (Claudiella, Lepicerus) as a shallow notch only. In Adephaga, it is usually absent, but a distinct cleft occurs in Haliplidae, Gyrinidae (Spanglerogyrus), Noteridae (Notomicrus) and some smaller Carabidae (Bembidion), while a shallow embayment is found in Trachypachidae. In Polyphaga, a distinct anal lobe is relatively common among basal polyphagan groups (Scirtidae, Eucinetidae, Calyptomerus, Derodontidae, some Hydrophilidae, incuding Helophorus and Hydrochus, Sphaeritidae, Synteliidae, Agyrtidae, Glaresidae, Trogidae etc.) and appears to be secondarily developed in small members of several more derived families.

\section{Wing folding and unfolding}

In our 1993 paper, we discussed the basal wing folding mechanism in Coleoptera, involving increase and decrease in the angle between the two main wing struts (RA and MP1+2), changes in the shape of the medial bridge, and rotation of both struts upon their axes (Kukalová-Peck \& Lawrence, 1993, Figs 93-95). We also noted that in-depth functional analyses would be necessary to clarify the mechanics involved. This clarification has been provided in a series of important functional studies by Fabian Haas and his co-workers. Haas \& Beutel (2001) clearly demonstrated with digital and high speed video sequences of a living tethered cetoniine scarab Pachnoda marginata Kolbe that the angle $\alpha$ between RA and MP1+2 is increased during unfolding and that both RA and MP1+2 also undergo some rotation about their axes. This is caused by the action of direct flight muscle M71 (M. pleuro-alaris), which rotates the third axillary, thus moving the medial basivenale, causing MA1+2 to move posteriorly. The radius anterior appears to be held in position by the basalar muscle M73 (M. sterno-basalaris). They also found that at least in Pachnoda the unfolding process described above was not reversible and movements of the elytra and abdomen were necessary to achieve folding. This last observation applies to some groups of beetles, but it is unlikely to represent the basal condition for the order. $P$. marginata is a derived member of the scarabaeid subfamily Cetoniinae a group noted for a highly specialized type of hind wing folding and unfolding. Furthermore, all scarabaeoid wings are derived with respect to basal staphyliniforms, such as Hydrophilidae.

According to Haas \& Beutel (2001, p. 140), the "highly variable kinematics of the hind wing unfolding excludes a mechanism relying significantly on stored elasticity" and in the Summary (Abstract) they further state that "Our findings are in clear contrast to the earlier assumption that the hind wings of Coleoptera either unfold or fold due to intrinsic elasticity." Haas et al. (2000) demonstrated the presence of resilin in the hind wings of Pachnoda and Coccinella using fluorescent and bright-field light microscopy, but they concluded that its probable functions 
were to reinforce major wing folds and provide the wing with some "elasticity in order to be deformable by aerodynamic forces". However, they did find a concentration of resilin in the vicinity of the medial bridge, which suggests to us the possibility that elasticity could be involved at least in the initiation of the folding process (anterior movement of MP1+2 and narrowing of the angle between it and RA).

The question of how an increase in the basal angle between RA and MP1+2 can affect the complex series of folds in the medial and apical fields was addressed by Haas \& Wootton (1996), who worked with mechanical and geometric folding models. By considering wing folding areas to consist of several groups of four solid panels rotating hingewise about four folding lines intersecting at a single point or knot, the entire unfoldingfolding process might be explained "as a series of linked levers, powered by the basal muscles, and openingclosing fold after fold in a chain reaction from the wing base to the tip". These authors admit, however, that to achieve both folding and unfolding other factors must also be involved.

Two important features of the wing folding mechanism, which have not been given sufficient attention are the bending zones and the folding loops, both defined and discussed in the previous section. We consider the radial and median bending zones and the radial and medial loops to be part of the groundplan of the coleopteran wing, in spite of the fact that in most beetle wings (those of most staphyliniform, elateriform and cucujiform Polyphaga) one or both have been lost or modified. When the two wing struts approach one another, they also rotate on their axes, causing their respective bending zones to curve towards one another; this process is aided by one or sometimes two radial cross-veins which join the radial loop to the medial loop. When the two bending zones are rotated in the opposite direction, in conjunction with the separation of the wing struts, then both are straightened out and neither is capable of bending; this causes the apical portion of the wing to unfold in the manner described by Haas \& Wootton (1996).

The occurrence of two bending zones is not a common feature in Coleoptera but occurs in some very basal groups of Polyphaga, such as Scirtidae, Eucinetidae, Nosodendridae, Dermestidae and Bostrichidae (Fig. 27). Among the Archostemata, Ommatidae has a welldeveloped radial bending zone, which is transformed into a hinge in Cupedidae and Micromalthidae. The medial bar in Archostemata has a very sharp bend crossed by a fold, which we consider to be homologous to the medial hinge of Adephaga and Myxophaga. The radial bending zone is particularly obvious in those wings with a very heavily reinforced radial bar, where deeply impressed crimping is necessary to allow bending to take place. These well-developed bending zones occur in many Adephaga and Staphyliniformia, but they are basically no different than those of Ommatidae, Scirtidae or Nosodendridae. We cannot agree with the statement by Beutel \&
Haas (2000) that this structure is absent from Archostemata and "the eucinetoid lineage" of Polyphaga.

\section{Veinal homologies}

Updating of veinal homologies in Coleoptera has been based on the recently published character table of Pterygota (Haas \& Kukalová-Peck, 2001). In the following sections, data enclosed in square brackets ([]) are from Kukalová-Peck \& Lawrence (1993).

Media [MA joins RP via the medial bridge]. As correctly shown in Trachypachus (Kukalová-Peck \& Lawrence, 1993, Fig. 17), MA joins RP immediately after leaving the medial basivenale (BM); the entry of MA into $\mathrm{RP}$ is membranized and discernible only in dissections. The medial bridge in Coleoptera is homologous with a long, sinusoid cross-vein brace rpma-mp near base in Megaloptera and Neuroptera (Figs 5-9), a strong, straight rpma-mp brace near base in Mecopterida: Diptera (Fig. 33), which is obscured when veins abat (Figs 10-18), and the fusion of RP + MA + MP at mid-wing in Hymenoptera.

Cubitus [The stem of $\mathrm{Cu}$ is absent; $\mathrm{CuA}$ starts directly from the cubital basivenale]. Within the Neoptera, the stem of $\mathrm{Cu}$ (fused $\mathrm{CuA}+\mathrm{CuP}$ ) is absent only in the Orthoneoptera (Fig. 2). In Coleoptera, the stem of $\mathrm{Cu}$ divides into a long, branched $\mathrm{CuA}$ and a short, curved $\mathrm{CuP}$, which ends on AA3 and encloses the $1^{\text {st }}$ cubito-anal cell. $\mathrm{CuA}$ forks into $\mathrm{CuA} 1+2$ and $\mathrm{CuA} 3+4$. $\mathrm{CuA} 1+2$ divides again, $\mathrm{CuA} 1$ forming a short fusion with MP4 (a long fusion with MP3+4 and MP4 in derived taxa), while $\mathrm{CuA} 2$ extends towards the posterior wing margin (Ommatidae, some Cupedidae and various Polyphaga) or is lost in various derived Polyphaga, as well as in Adephaga and Myxophaga). CuA3+4 forms a short, curved brace ending on AA3 and enclosing the $2^{\text {nd }}$ cubitoanal or wedge cell. Note that the fusion (brace) between the most-proximal branch of MP and the most-distal branch of $\mathrm{CuA}$, is shared by all Endoneoptera as a groundplan character.

Anal anterior $[\mathrm{AA} 1+2$ is present]. Among the Endoneoptera, AA1+2 is retained only in basal Hymenoptera; it is lost in Coleoptera + Neuropterida + Mecopterida (Figs 3-7, 8-10, 12-15, 17-18, 23-26). As discussed below, Blattoneoptera, Hemineoptera (Fig. 2), and Endoneoptera share a partial anojugal lobe starting at the anal fold and composed only of a branched AP and J. AA branches $\mathrm{AA} 1+2$ and $\mathrm{AA} 3+4$ join the remigium to form an extended flight unit and are progressively reduced. AA3+4 forks into two long, robust branches AA3 and AA4 in Coleoptera (Figs 23-26) and Megaloptera (Figs 7-8), bears several very short branches in Neuroptera (Figs 5-9), and is simple in Mecoptera and Trichoptera (Figs 10-17). In Coleoptera, AA3 receives two shortened, curved cubital branches, $\mathrm{CuP}$ and $\mathrm{CuA} 3+4$, which have been transformed into unique braces joining the AA area to the remigium (Figs 23-26). Coleoptera contain a relatively very large anojugal lobe that folds along the anal fold under remigium $+\mathrm{AA}$ as a single unit and like a book. This book-like folding occurs also in Paleozoic stem-line blattoneopterans and Isoptera (Mastotermes), 

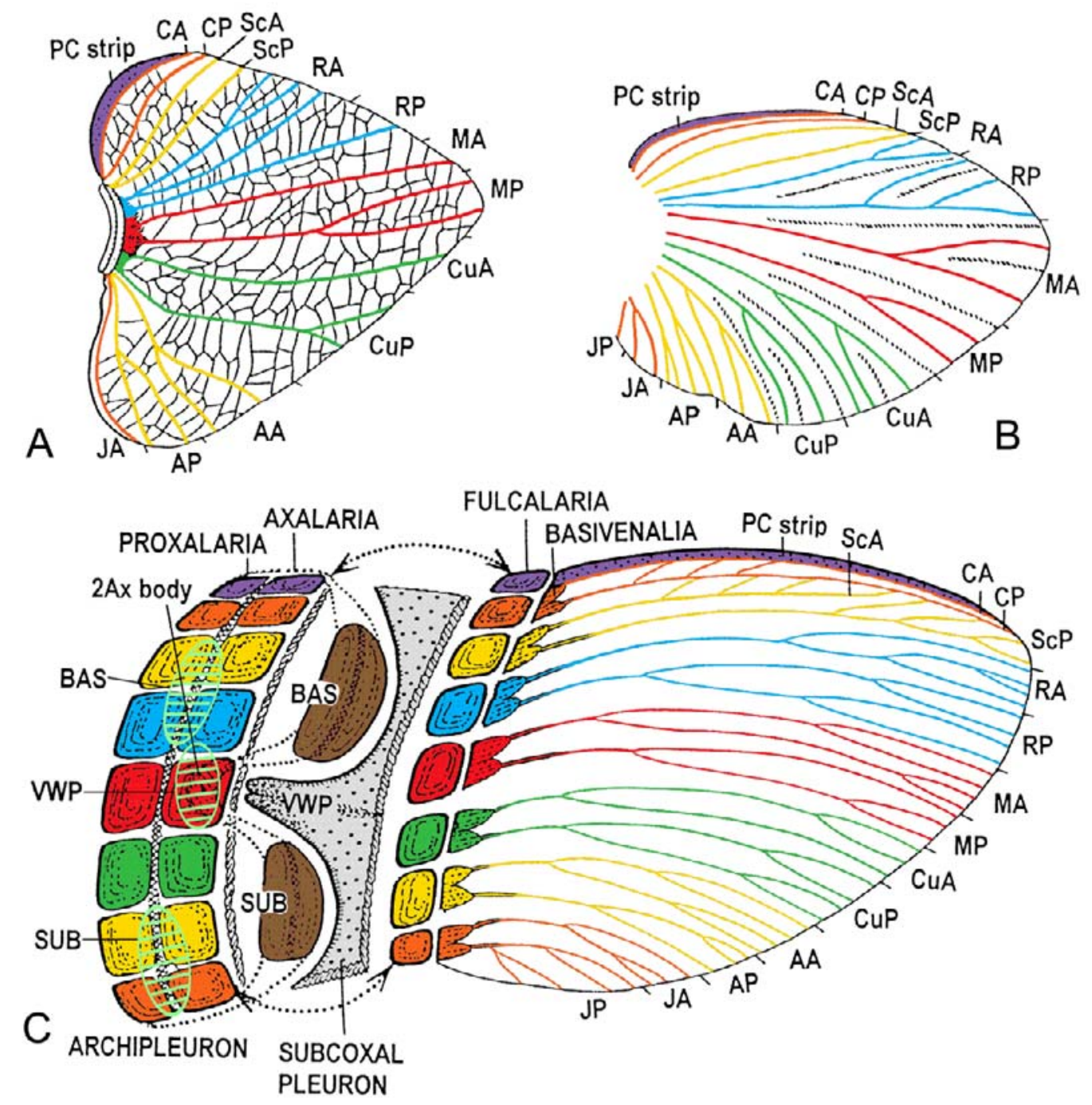

Fig. 1. Origin of the wing structure, evidence and hypothesis. A, B: The prothoracic wings of Carboniferous insects. A - Palaeodictyoptera: Stenodictya lobata (Brongniart, 1885) and B - Odonatoptera: Geroptera, undescribed species (Wootton \& KukalováPeck, 2000, Fig. 10). Prothoracic protowings, never adapted to flight, bore a complete set of branched veinal sectors (anterior A \& posterior P), which were basally fully separated (not fused into derived veinal stems of R, M, Cu), and lacked veinal braces. C. A composite of the most plesiomorphic veinal character states available in Pterygota (offered as protowing model by Kukalová-Peck in 1983) arrived at an identical veinal groundplan. Exploded diagram shows pteralia as the fragments of the subdivided archipleuron, which surrounded the flattened protowing. The fragments form proxalaria and axalaria above, and the basalare BAS, the ventral layer of $2 \mathrm{Ax}$ body above the ventral wing process VWP, and the subalare SUB under the protowing. Fulcalaria are probably fragments of the protowing base, and basivenalia the sclerotized veinal blood sinuses, each shared by two completely separated veinal sectors $\mathrm{A}$ and $\mathrm{P}$ (the stem of $\mathrm{R}, \mathrm{M}$ and $\mathrm{Cu}$ is missing). Light-green ovals indicate areas supported by pivots from BAS, VWP, SUB.

and it is indicated in basal Hymenoptera (all homoplasies, limited to those taxa with a partial anojugal lobe and with wings held flat in a dorsal position).

\section{Specific changes to figures in Kukalová-Peck \& Lawrence (1993)}

In most of the wing figures in the 1993 paper, changes must be made in the names of veins referred to in the preceding sections, in order to reflect new ideas on veinal homology. These changes in figure labels are given in Appendix 2.

\section{ORIGIN AND DIVERSIFICATION OF THE NEOPTERAN WING}

Most of the disagreements between our interpretation and evaluation of wing characters and those of Kristensen and others are rooted in a profoundly different concept of wing origin and diversification within the neopteran lineage. The sections below attempt to shortly review the available evidence applicable to the divergence of Pleconeoptera, Orthoneoptera, Blattoneoptera, Hemineoptera and Endoneoptera. 


\section{Wing development}

In experiments with insect larvae, the wing disc separates from the limb Anlage and occurs above and slightly posteriorly from the spiracle (Tower, 1903; Garcia-Bellido, 1975; Abouheif \& Wray, 2002; others). In the derived modern larvae, a small wing appendage evaginates and becomes larger, broader and flatter in each subsequent instar. The tergum, initially distant, gradually expands to meet the wing appendage (= wing bud plus pteralia) and a secondary fusion occurs in some basal taxa as late as in the 8th instar (in libellulid Odonata: Bocharova-Messner, 1959). In the plesiomorphic condition in the Carboniferous, all larval instars in the stem groups of Pleconeoptera and Hemineoptera had their wing pads fully articulated (not fused) with the tergum, but in the ancestral Blattoneoptera they were already fused and separated only by a suture. This shows that the tergum/wing pad fusion in the larvae is a secondary adaptation, which arose repeatedly and independently at different times and mostly after the end of the Paleozoic Era (Sharov, 1966; Kukalová-Peck, 1978, 1983, 1991, Figs 6.6A, B, 6.17; Shear \& Kukalová-Peck, 1990). Note that the wing appendage contains a veined wing plus all pteralia, including the anterior and posterior wing processes AWP and PWP (Fig. 1) (Stenzhorn, 1974, in transplant experiments). Therefore, the fusion between the wing processes AWP and PWP and the tergum, which repeatedly occurs in all Neoptera orders and at different taxonomic levels, should always be judged as a derived character (Haas \& Kukalová-Peck, 2001).

\section{Origin of wing venation (Fig. 1)}

In Arthropoda, the limb-derived, locomotory plate-like appendages, such as the crustacean swimming uropods (or insect wings), are nourished by blood channels expressed on the surface as sclerotized ridges, which reinforce the plate by dividing repeatedly and dichotomously and filling the entire space. The ridges/veins are basally separated (not fused into veinal stems) and are not connected by any veinal or cross-vein braces. A reconstruction of the insectan protowing based on the least fused and braced character states in modern flying wings, revealed the same veinal pattern (Kukalová-Peck, 1983). Nearly two decades later JKP realized that principal veins expressed by two independent veinal sectors (anterior A and posterior $\mathrm{P}$ ) were also recorded in the prothoracic wings of Carboniferous Palaeodictyoptera and Geroptera (basal Odonatoptera) (Fig. 1A, B) (Kukalová-Peck, 1978, 1991; Shear \& Kukalová-Peck, 1990; Wootton \& Kukalová-Peck, 2000). Thus, prothoracic wings, which never adapted to a powered flapping flight, appear to have retained a near-protowing venation (i.e., without veinal stems and braces) in spite of the evident diversification of the associated flight-adapted wings into different orders (!) (Kukalová-Peck, 1997; Haas \& Kukalová-Peck, 2001).

Numerous experiments with wing models repeatedly showed that the flapping aerial flight is not possible without a relatively large wing foil with a reinforced ante- rior margin, asymmetrically arranged veins supporting the anterior margin, and veinal fusions and braces near wing base (Wootton, 1976, 1979; Brodsky, 1994). This shows that the prothoracic wings of Carboniferous insects were adapted for some kind of movement but not for flapping aerial flight. The protowing veinal states suggest that (1) the precosta PC formed precostal strip fused with costa anterior CA into anterior margin; (2) the following veinal sectors were primitively branched: $\mathrm{CP}$; ScA, ScP; RA, RP; MA, MP; CuA, CuP; AA, AP; JA, JP; (3) all fusions of veinal sectors basally into veinal stems, cross-vein braces and fusion braces between the veinal branches, are derived; (4) pectinate branching, reduced branching, and intercalary veins formed from the network between veins are derived.

\section{Origin of wing articulation (Fig. 1C)}

On the insectan thorax, two limb segments become flattened into plates and imbedded into the pleural membrane: the epicoxal pleuron (archipleuron) and the subcoxal pleuron. In modern insects the plesiomorphic plate-like state of the archipleuron is best preserved in the prothorax of Archaeognatha (Kukalová-Peck, 1997, Fig. 19.5a) and is placed at the same level as the wing articulation, between tergum and subcoxal pleuron, above the spiracle. The origin of the wing sclerites by fragmentation of this plate is also indicated by a consistent occurrence of very dense, plate-like dorsal pteralia in the Carboniferous Palaeodictyoptera (Kukalová-Peck \& Richardson, 1983), Megasecoptera, Permothemistida, Geroptera, Odonatoptera and Ephemeroptera (neopteran pteralia are not suited for fossilization) (Kukalová-Peck, 1983, 1991, 1997). The fragments or pteralia are aligned in two directions: (a) proximodistally, forming a row above each of the eight blood channels aligned with the eight principal veins and named according to veinal symbols; and (b) anteroposteriorly, arranged in two columns - a proximal one containing the proxalaria (PR) and a distal one with the axalaria (AX). As supporting evidence, the thoracic subcoxal pleuron in pterygotes, silverfish and Diplura subdivides in a similar way. The next two columns of pteralia, the fulcalaria (F) and basivenalia (B) belong to the wing appendage, the former being derived from the ring-like protowing base on the body side of the articulation and originally provided with musculature, and the latter being veinal blood sinuses on the wing side of the articulation and always lacking muscles. The protowing was supported by a series of pivots from the ventral wing process (VWP) on the subcoxal pleuron, plus the basalare (BAS) and subalare (SUB). Although all of the pteralia - PR, AX, F and B - are homologous in Neoptera and Paleoptera, they became associated in a number of different ways to form very different composite sclerites (Kukalová-Peck, 1983, 1997; see Haas \& Kukalová-Peck, 2001, Figs 1a, b, c for a color-coded homologized articulation in Neoptera). The basal pteralia are better preserved in lower Neoptera, and their reduction, fusion, enlargement, partial membranization, or absence is considered derived. Note that the complete independence of the wing from the tergum and the ability of wings to be moved 
unimpeded including being flexed backwards (as in fossil juveniles and adult Neoptera and one extinct order of Paleoptera) are considered to be plesiomorphic.

\section{Wing shifting and the origin of the basalare, subalare and ventral $2 \mathrm{Ax}$ body (Fig. 1C)}

The wing appendage almost certainly shifted upwards and into the epicoxal pleuron (archipleuron), which then closed beneath it (as coxa does in a similar way beneath the coxal exites in Archaeognatha; Kukalová-Peck, 1987, 1997, Fig. 19.3e). The wing appendage then broadened into a plate-like appendage surrounded by a flattened epicoxal pleuron (Fig. 1C). The ventral part of the archipleuron fragmented into three pteralia, the basalare, the subalare, and the ventral part of the second axillary body, which lies just above the ventral wing process or main wing pivot.

\section{Wing homologues in other Arthropoda}

Existing evidence is consistent with the wing appendage originating from an articulated, mobile, flattened epicoxal exite (outer branch) of the epicoxal limb podite. This first of the limb podites was flattened in basal Arthropoda into a pleural plate (epicoxal pleuron or archipleuron) and was the site of articulation for the basal limb composed of $\mathbf{1 0}$ cylindrical podites (Kukalová-Peck, 1983, 1997). The protowing-exite has been functional since the Precambrian, most probably initially as an aquatic plate gill. Genetic evidence suggests that the insect wing is homologous with dorsal gills of primitive freshwater branchiopod Crustacea (Averof \& Cohen, 1997) and other arthropods (Abouheif \& Wray, 2002; Shubin et al., 1997).

\section{Wings in the apterygotes}

At least four early insectan lineages diverged from the basal free-ranging ancestral condition and became adapted to a cryptic life style in confined spaces, thus losing or transforming their protowing appendages: the Archaeognatha (jumping bristletails) and the closely related fossil order Monura, the pterygote sistergroup Zygentoma (silverfish) and the distantly related fossil order Cercopoda (Kukalová-Peck, 1997). All four groups living in this less varied enclosed environment retained many primitive features that vanished in the free-ranging Insecta. Archaeognatha and Monura lost the protowings and laterally expanded their terga, but retained the plesiomorphic (plate-like, not fragmented) archipleuron (present in the prothorax of modern bristletails and in all thoracic segments of fossil monurans), the original cylindrical state of the subcoxa in the meso- and metathorax, and the full 10-segmented arthropodan limb in the maxillary palp (Kukalová-Peck, 1987, 1997). In Pterygota all thoracic subcoxae are derived, flattened into subcoxal pleura and equipped with a ventral wing process, on which the wing base rests and pivots. In Zygentoma, the sister-group of Pterygota, the protowings were transformed into protective side lobes, which look superficially like "tergal lateral outgrowths", but which harbour inside them tracheae branching in a typical pterygote wing pattern (Šulc, 1927). The three pairs of thoracic flattened subcoxal pleura in silverfish became desclerotized to accomodate a highly autapomorphic, enhanced leg stride (Manton, 1977). In spite of the fact that apterygotes are in many characters more primitive than pterygote insects, they also bear many derived characters highly adapted to a cryptic lifestyle, including a replacement of articulated protowings with protective thoracic side lobes. The sidelobes of Archaeognatha and Zygentoma are thus non-homologous secondary adaptations and do not provide evidence that insect wings evolved from the lateral tergal outgrowths (= paranota). The paranotal hypothesis of wing origin built on the misinterpreted apterygote sidelobes was favored by Snodgrass (1935), and more recently revived by Willmann (1997), Hörnschemeyer (1998, 2002) and Wheeler et al. (2001), without generating any good characters. But it has been falsified by ever increasing amount of evidence from paleontology, embryology, developmental genetics and transplants, as reviewed above.

It follows that a successful use of character-rich wing venation and articulation in the character-starved phylogeny of higher taxa depends in every aspect on the allimportant correct protowing model: for complete homologization, reconstruction of the reliable higher-level groundplans, finding characters, evaluation of all character states, and for providing the dependable synapomorphies.

\section{Neoptera: basal divergence in wing structure}

Neoptera differ from the sistergroup Paleoptera in 65 homologized wing characters (Haas \& Kukalová-Peck, 2001, pp. 492-496). Neoptera are best defined by their distinctly heteronomous wing pairs, and their unique, complex wing articulation. In early Neoptera, the fore wings were narrower than the hind wings and had a dual function: they were used for flight but also for protecting the much thinner and broader hind wings. The hind wings in modern basal Neoptera lineages (Pleconeoptera, Orthoneoptera, Blattoneoptera) are powered by a stronger flight musculature and function as the main flying pair (Brodsky, 1994). The protective fore wings are more or less tegminous and have an asymmetrical veinal pattern, with the anal area basally reinforced by a thick anal bar, anal branches AA $1+2$ and AA3 +4 widely spaced, AA $3+4$ and AP shortly fused basally, and the jugal area strongly diminished, sunken and folded beneath the wing. The hind wings are more plesiomorphic than the fore wings in having veins radially arranged as in the protowing, and the jugal area is placed at the same level as the rest of the wing (Haas \& Kukalová-Peck, 2001). Thus, in all Neoptera (including Strepsiptera, Fig. 28), the fore and hind wing pair can be instantly recognized by a different arrangement of veins and areas (Kukalová-Peck, 1997).

An especially meaningful attribute of the Neoptera wing groundplan for the higher phylogenetics is its complete absence of veinal stems or braces. The stem of $\mathrm{R}$ is absent in hind wings of fossil pleconeopterans and hemineopterans (Kukalová-Peck \& Brauckmann, 1990, Figs 32-34), Strepsiptera and basal Coleoptera (Figs 
28-31). The stem of $\mathrm{M}$ is absent in all Endoneoptera, Hemineoptera and Blattoneoptera (Figs 2-18, 28-33). The stem of $\mathrm{Cu}$ is absent in Orthoneoptera (Fig. 2A, B; J. Kukalová-Peck \& D. C. Rentz, unpublished). The crossvein brace mp-cua (arculus) is present in both wing pairs only in Hemineoptera, Endoneoptera and Dermaptera. It is absent in both wing pairs of Orthoneoptera (Fig. 2), of the extinct stem groups of Pleconeoptera (Liomopterida) and Blattoneoptera, and of fossil and modern Isoptera. In modern Blattodea and Mantodea, mp-cua is limited only to the hind wings. Modern Plecoptera contain instead of mp-cua an analogous brace m-cua (Fig. 2A, B), (Carpenter, 1992; Haas \& Kukalová-Peck, 2001, Fig. 20). Therefore, no matter how frequently it occurs, the cross-vein brace mp-cua cannot be part of the Neoptera groundplan. The above, scattered pattern of veinal fusions into stems and braces needed for powered flight indicates that they developed in the superordinal lineages gradually, independently and in parallel and are not shared with the allNeoptera groundplan. The fossil record shows a much slower evolution of flight in Neoptera than in Paleoptera. The first superior neopteran fliers, Diptera, some Hymenoptera, and some Lepidoptera (Sphingidae) occurred as late as in the Upper Triassic, Jurassic and Cretaceous. In contrast the Paleoptera, the wing groundplan of which includes two fused veinal stems (the stem of $\mathrm{M}$ and of $\mathrm{Cu})$ and a brace between anal veins, produced the first superior fliers Megasecoptera and Odonatoptera in the Carboniferous, some 100 million years earlier than in Neoptera (Carpenter, 1992; Shear \& Kukalová-Peck, 1990; Wootton \& Kukalová-Peck, 2000).

When flexed back over the abdomen, the neopteran wings became folded and locked in the resting position by the $3^{\text {rd }}$ axillary, which rotated, pulled on the cubital, anal and jugal arms articulated with the corresonding basivenalia, and subsequently collapsed into the articular membrane. The movements of the flight musculature and the terga were transferred to the wing by two obliquely constructed composite sclerites, the first and second axillaries, which provided space for the third axillary to rotate. The combination of highly sophisticated flexinglocking mechanism with slowly improving flying ability suggests that the early Neoptera probably flexed their wings and hid from predators in narrow spaces or went into free-fall, rather than flying away. A similar escape strategy occurs also in Coleoptera.

Different character states contained in the hind wings of major neopteran lineages. The early neopteran hind wings developed two different kinds of fusions of the medial sectors MA and MP, two differently constructed anojugal lobes, and two ways in which anojugal lobes are proximally articulated to the tergum: one set of characters occurs in Orthoneoptera + Pleconeoptera and the other in Blattoneoptera + Hemineoptera + Endoneoptera.

(i) The stem of $M \&$ the full anojugal lobe. Pleconeoptera (including Embioptera) and Orthoneoptera (including Phasmatodea) (Fig. 2) share the stem of $M$ (MA + MP fused) (an apomorphy) and the full anojugal lobe supported by all branches of the anal and jugal systems, dichotomously divided and radially arranged: $\mathrm{AA} 1+2, \mathrm{AA} 3+4, \mathrm{AP} 1+2, \mathrm{AP} 3+4, \mathrm{JA}, \mathrm{JP}$ (a plesiomorphy). Distally, the full lobe is separated from the remigium by the claval flexion line (a plesiomorphy). Proximally, the jugal veinal system is articulated with the tergum or the posterior wing process by secondary struts (an apomorphy), while the 3Ax jugal arm is completely lost (Fig. 35).

(ii) MA fused with radius \& the partial anojugal lobe. Blattoneoptera, Hemineoptera and Endoneoptera (Figs 2-15, 23-26, 28) share the condition of MA being fused basally with the radius and diverging apically from RP (an apomorphy), and bear a partial anojugal lobe, which is supported only by branches of AP1+2, AP3+4, JA, JP (an apomorphy). The branches AA1+2 and $\mathrm{AA} 3+4$ joined the remigium to form an extended flight unit and occupy an increasingly narrower area (an apomorphy). Distally, the partial lobe starts at the anal fold and the claval flexion line becomes progressively shallower and shorter (an apomorphy). Proximally, jugal basivenale of the jugal veinal system is articulated with the $3 \mathrm{Ax}$ jugal arm manipulated by rotating 3Ax (Fig. 35) (a plesiomorphy).

A similar division into Orthoneoptera + Pleconeoptera and Blattoneoptera + Hemineoptera + Endoneoptera is in the composition of the ovipositor. In a basal pterygote ovipositor, fully homologized with the arthropod groundplan limb (Kukalová-Peck, 1991, Fig. 6.9B, 6.10E), the 3rd valvula in Neoptera appears to be derived from two different sources. In Orthoneoptera and Pleconeoptera (ovipositors known in fossil pleconeopterans) it is formed by a sclerotized gonostylus (Sharov, 1966, Fig. 76; Hennig, 1981; others). In Blattoneoptera (ovipositors known in the penultimate nymph of Blatta orientalis), Hemineoptera and Endoneoptera, the $3^{\text {rd }}$ valvula is formed by an elongated gonocoxite (Sharov, 1966, Fig. 77; Hennig, 1981; Kukalová-Peck, 1991, Fig. 6.10E).

Blattoneoptera (Fig. 2) retained the plesiomorphic, protective fore wings and broader, thinner hind wings, but their partial anojugal lobe is supported by fewer primary veins. To become air-worthy, the anojugal fan in Blattoneoptera compensated by becoming enlarged (sometimes enormously) and by filling out the extra wing membrane with secondary, pectinate, intercalary branches of the anal vein AP (in Dermaptera, Mantodea and many Blattodea).

Hemineoptera and Endoneoptera (Figs 2, 3-18). In this group, evolution took quite a different path towards flight improvement. The hind wings became gradually smaller, and the larger fore wings became the main flying pair. Many wings lost most of the ancestral veinal branches and cross-veins, added numerous setae, several fusion braces and cross-vein braces, and a more or less transparent membrane, improving their flying ability. Eventually, small hind wings in some groups became hooked to the fore wings by lobes, setae or hamuli, and the two pterothoracic wings on each side began to function as a single, extended flight unit. As documented in 

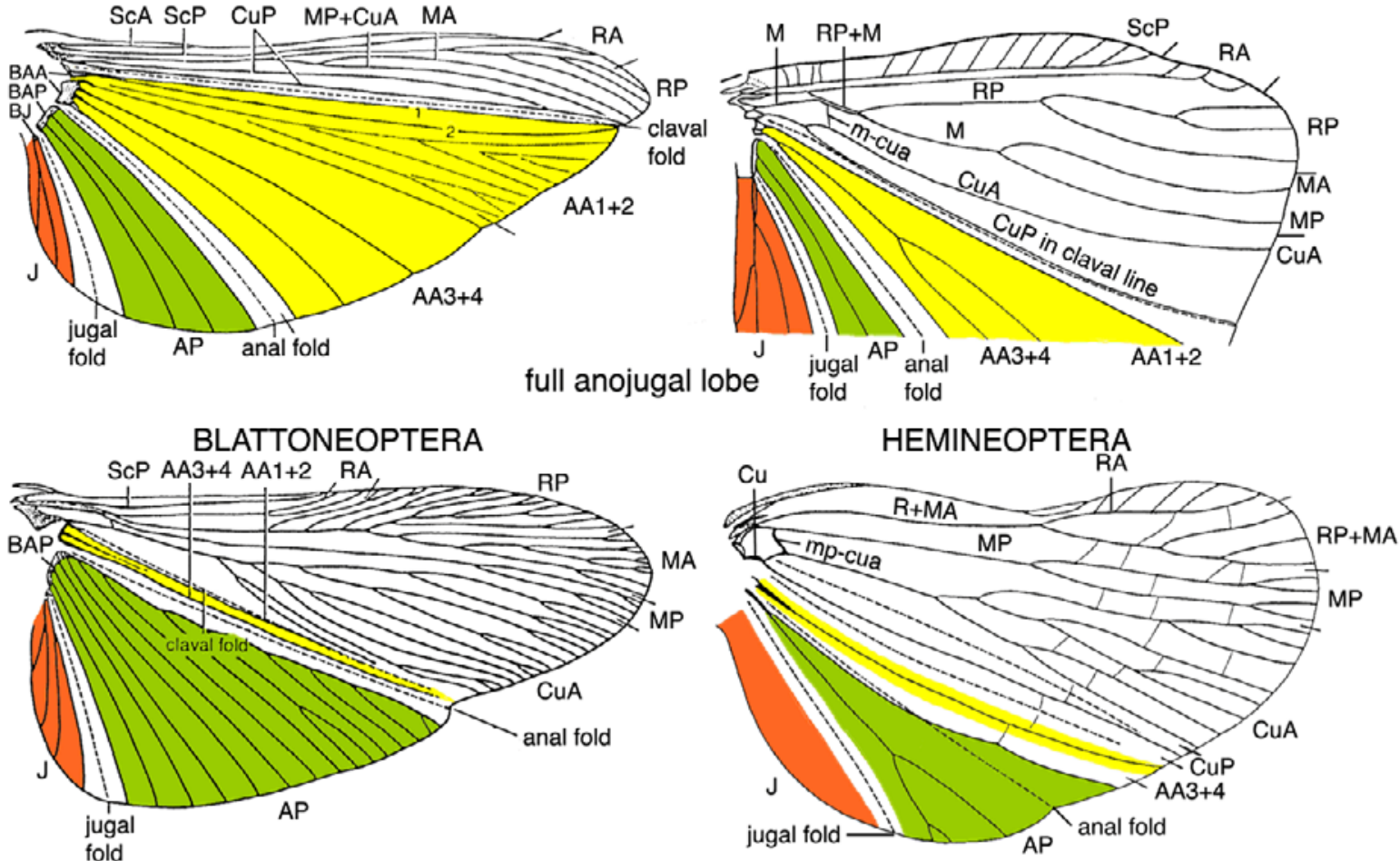

partial anojugal lobe

B

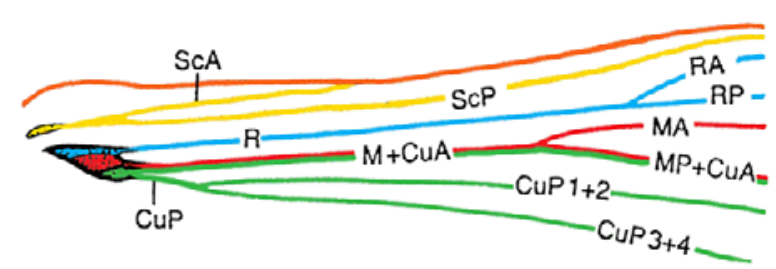

BLATTONEOPTERA

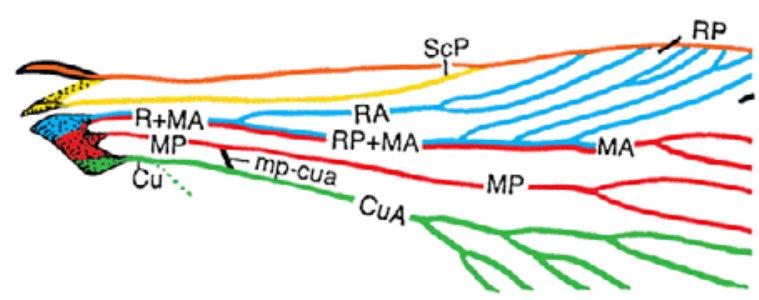

\section{PLECONEOPTERA}

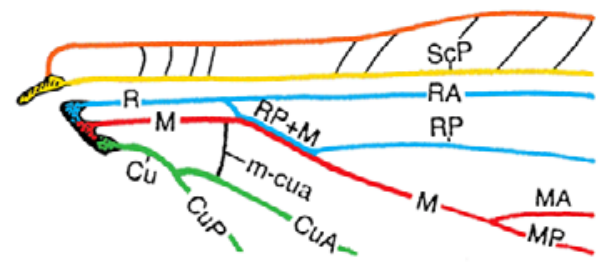

HEMINEOPTERA

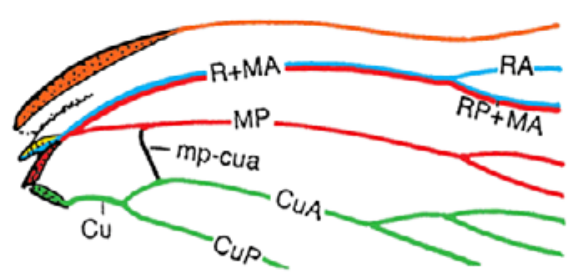

Fig. 2. Neoptera hind wings: two groundplans of the basal division. A - two kinds of anojugal lobe. Orthoneoptera + Pleconeoptera share the full anojugal lobe including a large AA area, and starting at the claval flexion line (= claval fold). Blattoneoptera + Hemineoptera (+ Endoneoptera) share the partial anojugal lobe including a narrowed AA area combined with the remigium, and starting at the anal fold; B - two kinds of MA fusion. In Orthoneoptera + Pleconeoptera MA fuses basally with MP into MA + MP, a derived fusion called the stem of $\mathrm{M}$ (in Orthoneoptera, $\mathrm{M}$ is joined also by $\mathrm{CuA}(\mathrm{CuA}+\mathrm{M}+\mathrm{MP})$, with $\mathrm{CuA}$ separating apically from MP. In Blattoneoptera + Hemineoptera (+ Endoneoptera), MA fuses basally with R and RP into MA + R + RP, and MA separates again apically from RP. Orthoneoptera: Caelifera: Acrididae: Valanga irregularis (Walker, 1870); Pleconeoptera: Eustheniidae: Eusthenia sp.; Blattoneoptera: Blattidae: Periplaneta americana (Linnaeus, 1758); Hemineoptera: Fulgoromorpha: Eubrachidae: Eubrachys sp.

many experiments (Brodsky, 1994; Wootton, 1979, 1981, 1990, 1992, 1995, 1996; Wootton \& Kukalová-Peck, 2000), the most probable motivation for these partly shared and partly parallel transformations was survival through an improved flying ability. 
Coleoptera evolved very differently from the other Endoneoptera, by developing a unique survival and escape strategy, largely analogous to that of the early Neoptera. The fore wings were transformed into shortened, convex, and strongly sclerotized elytra adapted to protect the much larger and thinner hind wings. To lift the relatively heavy bodies, the hind wings became broadened, greatly elongated and equipped with innovative secondary veinal support; instead of adding intercalary branches (as in blattoneopterans), RP branches, MP1+2 and $\mathrm{Cu}$ became flanked by broad, flat sclerotized strips of cuticle and propped by long, strengthened cross-vein braces. The anterior margin was strengthened proximally by the annexation of ScP and RA to form the broad radial bar, and the greatly enlarged apical field was filled out by special, curved branches of RP. The hind wings were folded at rest beneath the elytra by means of an unusual type of apical folding involving two veinal loops joined by one or two cross-veins and a pair of one-way bending zones, allowing the reinforced wing struts to bend towards one another. Remarkably, the enlarged yet poorly supported anojugal lobe is folded under the remigium along the anal fold as a single unit (like a book rathern than a fan), as in the ancestral Paleozoic blattoneopterans and modern termites of the genus Mastotermes.

\section{PHYLOGENETIC ANALYSIS OF THE MAJOR GROUPS OF ENDONEOPTERA AND THE SUBORDERS OF COLEOPTERA WITH THREE BASAL NEOPTERAN OUTGROUPS}

The veinal system in Coleoptera cannot be understood (and used in higher phylogenetics) without being thoroughly compared with those of other endoneopteran orders. The basal veinal system of Endoneoptera is the end product of a complex series of fusions, braces and reductions, which can be clearly judged only by starting at the Neoptera level and following the entire path in which these derived character states were added and accumulated. In the following phylogenetic analysis we have included the four Coleoptera suborders, four other endoneopteran taxa and three basal neopteran subdivisions as outgroups.

\section{Included taxa}

Orthoneoptera (Fig. 2). This subdivision, containing the groups Ensifera, Caelifera and Phasmatodea, was chosen as an outgroup representing those Neoptera having plesiomorphic wing pairs (fore wings tegminous, narrow, hind wings broad, thin, functioning as the main flying pair), and most veinal characters plesiomorphic at the Neoptera level [ScA and ScP long, branched; RA and RP branched; MA not fused with R or RP at wing base; MA and MP branched; the stem of $\mathrm{Cu}$ absent (veinal sectors $\mathrm{CuA}$ and $\mathrm{CuP}$ separate, not fused basally into a derived stem of $\mathrm{Cu}$ ); $\mathrm{CuA}$ and $\mathrm{CuP}$ dichotomously branched; anojugal lobe (here identified as "full anojugal lobe") starting at a deep claval furrow serving as a flexion line; anal fold resembles other folds; all anal veins present and fully branched; jugal veins fully branched; and numerous irregular cross-veins present]. Two widely dis- tributed cross-vein braces, m-cua (in Plecoptera and Embioptera: Kukalová-Peck, 1991, Fig. 6.19 A) and mp-cua (arculus, in fore- and hind wing groundplan of Hemineoptera + Endoneoptera, and the hind wings of some Blattoneoptera) absent, but replaced with another, much longer, autapomorphic fusion $\mathrm{CuA}+\mathrm{M}+\mathrm{MP}$ (CuA separating apically from MP). Note that the orthoneopteran wing venation has been newly homologized by JKP and D.C.F. Rentz (unpublished) and the brace "mp-cua" previously believed to be present in many orthoneopterans, was recognized as the anterior branch of CuP (Fig. 2A, B). The related subdivision Pleconeoptera, including the modern orders Plecoptera and Embioptera, shows many autapomorphic veinal reductions and unique folds in the anojugal area. Therefore, Pleconeoptera has not been included in the analysis below.

Blattoneoptera (Fig. 2). This subdivision contains the orders (Grylloblattodea + (?Zoraptera + (Dermaptera + $($ Mantodea $+($ Blattodea + Isoptera $))))$ ) (Haas \& Kukalová-Peck, 2001). It is the most basal assemblage among those Neoptera bearing a partial anojugal lobe and MA fused basally with R (Blattoneoptera + (Hemineoptera + Endoneoptera)). The hind wing often bears a secondarily enlarged anojugal lobe supported by secondary pectinate branches on AP. Brace mp-cua (shared by Hemineoptera + Endoneoptera) is absent in ancestral Blattoneoptera, modern Isoptera and in the fore wings of Blattodea and Mantodea, but present in both wing pairs of Dermaptera. Therefore, it is almost certainly not part of the Blattoneoptera groundplan. Dermaptera and some cockroaches (Blatellidae) developed an apical folding, which is analogous to that in Coleoptera. Paleozoic ancestral blattoneopterans, Isoptera and Coleoptera fold their anojugal lobes flat and book-like along the anal fold.

Hemineoptera (Fig. 2). This subdivision, the sister group of Endoneoptera, includes the orders Psocodea, Thysanoptera, Phthiraptera, Sternorrhyncha, Coleorrhyncha, Heteroptera, Fulgoromorpha and Cicadomorpha. Only in Fulgoromorpha (Fig. 2) and some Cicadomorpha is the hind wing venation complete enough to be compared with endoneopteran venation. The hind wing groundplans of Hemineoptera and Endoneoptera are compared by Haas \& Kukalová-Peck (2001, Table 6).

Endoneoptera (Figs 3-35). This subdivision includes the order Hymenoptera and the superorders Neuropterida, Mecopterida and Coleopterida. Endoneopteran hindwing groundplan characters offered by Haas \& Kukalová-Peck (2001, pp. 498-500, 504-506) have been refined here as follows. Brace mp-cua (arculus), often believed to be ubiquitous in Neoptera, actually occurs in both wing pairs only in Endoneoptera + Hemineoptera, and in the order Dermaptera. Its presence in Pleconeoptera and Orthoneoptera is an error in homologization (see above). Crossvein brace rpma-mp near wing base occurring in Coleoptera + Neuropterida + Mecopterida may be expressed as ma-mp brace (in some Neuropterida) or as rma-mp brace (in Mecopterida, Diptera). This important brace forms in Coleoptera the medial bridge, in Neuropte- 
rida a long sinusoid cross-vein, in Mecopterida: Diptera a strong oblique bar, and in Mecoptera, Trichoptera and Lepidoptera it is replaced by stiffened membrane connecting the veins. In Hymenoptera, the brace is absent but there is instead at mid-wing an extensive and partly membranized veinal fusion RP + MA + MP. Endoneopteran apomorphic fusion brace MP4 $+\mathrm{CuA} 1$ near the posterior wing margin is a veinal fusion between the utmost posterior branch of $\mathrm{MP}$ and utmost anterior branch of $\mathrm{CuA}$, which can involve MP4 $+\mathrm{CuA} 1+2$ or $\mathrm{MP} 3+4+\mathrm{CuA} 1+2$ as a derived alternative. This fusion brace, once considered possibly present in the Diptera and Lepidoptera (Hennig, 1981), is here documented also in Hymenoptera, Coleoptera, Neuropterida, Mecoptera and Trichoptera (Figs 1-17, 23-26).

Hymenoptera (Figs 3-4, 34K, 35K). This order contains a unique mix of plesiomorphic and autapomorphic veinal attributes, most fully expressed in the family Xyelidae. Hymenoptera appear to share no wing synapomorphies with either Coleoptera, Neuropterida or Mecopterida. They also exhibit several plesiomorphies at the Endoneoptera level which are absent in the other groups: a relatively large and broad hind wing; a perfectly preserved, long claval furrow; AA1+2 retained (weakly preserved in blattoneopterans but lost in Hemineoptera and again in Coleoptera + Neuropterida + Mecopterida); relatively broad AA area; and short stem of $\mathrm{Cu}$. Autapomorphies are numerous and pronounced: presence at midwing of a "Y" shaped fusion brace of RP + MA + MP in the fore wing (Fig. 3), suggesting that this fusion has also occurred in the hind wing but has become membranized (compare Figs 3 and 4); RP reduced to a single vein; MP forming three long, zig-zag fusions, $\mathrm{MP}+\mathrm{CuA}$ (arculus), $\mathrm{RP}+\mathrm{MA}+\mathrm{MP}$ (analogue of the rpma-mp brace) and $\mathrm{MP} 3+4+\mathrm{CuA} 1+2$; weakened or reduced link between $\mathrm{Cu}$ and MP; loss of $\mathrm{CuP}$ except for a small basal remnant (Fig. 3); reduction of serial cross-veins to several prominent, long, stabilized cross-vein braces; and transparent wing membrane. This highly unusual mix of many deep plesiomorphies and highly derived, exceptional autapomorphies in a superbly flight-adapted groundplan, signals a very early divergence from the endoneopteran stock followed by an intense and rapid flight adaptation. Note that the peculiar arrangement of veins in the AP area is reminiscent of that in Coleoptera.

Neuropterida (Figs 5-9, 34N, O, 35N, O). This group contains Neuroptera, Megaloptera and Raphidioptera. Superorder Neuropterida, or sometimes the order Megaloptera alone, is usually considered to be the sistergroup of Coleoptera; however, we could not find any wing synapomorphies supporting such a relationship (see analysis). We document Megaloptera by Corydalidae (Figs 7-8), Neuroptera by Ithonidae and Dilaridae (Figs 5-6) and Raphidioptera by Raphidiidae (Fig. 9). Long, undulated brace rpma-mp (Figs 6, 7, 9) may be expressed as ma-mp brace (Fig. 5). Fusion MP4 + CuA1 is inconspicuous in modern Neuropterida (crossing of $\mathrm{CuA}$ towards MP may ressemble a weak cross-vein), but it is easily recognizable in Mesozoic fossil Neuroptera (JKP, unpublished). Note that $\mathrm{AA} 1+2$ is present in the fore wing but lost in the hind wing, which has a partial anojugal lobe.

The wings of Megaloptera (Figs 7-8) very probably increased in size only after they lost many veinal branches. A similar enlargement accompanied by veinal loss in Coleoptera is probably responsible for the superficial similarity of the hind wings in these two groups. Raphidioptera (Fig. 9) share their impoverished veinal system with Megaloptera (compare Figs 9 and 7-8), but several autapomorphies are added: the anterior wing margin stiffened by the arched end of ScP; reduction of cross-veins to a few enhanced cross-vein braces; many veins with a zig-zag course; short intercalar branches close to the wing margin forming broad forks; and hyaline wing membrane.

Frequent errors have been made in veinal homologization. In Neuroptera, the base of RP at its separation from MA is often reduced and RP becomes connected to RA by one of the oblique ra-rp cross-veins, which is then misinterpreted as the base of RP. In Raphidioptera, the undulated rpma-mp cross-vein brace was misinterpreted as a principal vein by Willmann (1994). For a fully homologized neuropterid venation, see Figs 5-9.

Mecopterida (Figs 10-14, 32-35). This section of Endoneoptera includes two superorders, Antliophora and Amphiesmenoptera. Antliophora (Mecoptera, Siphonaptera, Diptera) are documented by the mecopteran taxa Notiothaumidae (Figs 10-11), Panorpidae (Figs 13-14), Choristidae (Fig. 12), and by the dipteran taxa Tabanidae (Fig. 33) and Tipulidae (Figs 34R, 35Q). Amphiesmenoptera (Trichoptera, Lepidoptera) are documented by the trichopteran families Polycentropodidae (Fig. 15) and Philopotamidae (Figs 16, 17), and by the lepidopteran family Neopseustidae (Fig. 18). Mecopterida is usually considered to be the sister group of Hymenoptera, but this relationship is not supported by our analysis (see p. 34). The mecopterid-neuropterid wing synapomorphies, especially those involving the RA-RP system, stand out most clearly in basal genera, such as Notiothauma (Figs 10-11) and Ithone (Fig. 5). The costal area in Notiothauma bears the most plesiomorphic ScP branches in Endoneoptera. The anal area in Neuropterida shows AA3+4, AP1+2 and AP3 +4 repeatedly branched; in Mecopterida the anals are similar but impoverished. Note that AA1+2, present in the fore wings, is lost in the hind wings, which have a partial anojugal lobe.

In Antliophora, MA does not separate apically from $\mathrm{RP}$, brace rma-mp forms a bar-like cross-vein (in Diptera; replaced by veinal contact in Mecoptera), brace mp-cua is a cross-vein, $\mathrm{CuA}$ is bifurcate, $\mathrm{CuA} 1+2$ fuses with the utmost posterior MP branch near the posterior margin, and the extensive narrowing and petiolation leads to loss of anojugal branches. The typical short apposition of $\mathrm{CuP}$ to $\mathrm{AA} 3+4$ is also found in Miomoptera, the ubiquitous Carboniferous and Permian extinct order of small insects of uncertain affinity (Carpenter, 1992). This identifies some Miomoptera very probably as a member of the ant- 
liophoran stem group. We found no antliophoran wing characters in Strepsiptera.

In Amphiesmenoptera, $\mathrm{ScP}$ is less shortened, the costal area is usually broader, MA separates visibly from RP (Figs 15-18), mp-cua is a short cross-vein brace or fusion brace, and $\mathrm{CuA}$ is 3-branched and less impoverished (all plesiomorphies with respect to Antliophora). Brace rma-mp is replaced by a strip of stiffened membrane (Fig. 15 ), and fusion of MP4 with $\mathrm{CuA} 1+2$ is very long (two autapomorphies). The ra-rp cross-veins are few, but in basal groups they may be slanted backwards (Figs 15, 17) as in Neuropterida. Note that in Lepidoptera several of the Mecopterida-level characters have become reduced or lost; the RA-RP system is no longer similar to that in Neuropterida, $\mathrm{CuP}$ is highly reduced, and the crossing of $\mathrm{CuA} 1+2$ to $\mathrm{MP} 3+4$ (preceding its fusion with MP3+4) and the $\mathrm{AA} 3+4$ bulge towards $\mathrm{CuP}$ have been almost always lost (Fig. 18).

Coleopterida (Figs 19-31, 34L, 35L). This subdivision includes the orders Strepsiptera and Coleoptera discussed below.

Strepsiptera (Figs 28-30, 34M, 35M). Strepsiptera are minute, highly modified parasites whose origins are obscured by reduction combined with extreme specialization. Strepsiptera share with Coleoptera a large number of unique wing synapomorphies resulting from the cladistic analysis below; these are discussed in detail in the section dealing with characters (pp. 112-128) and in the explanation to Figs 28-35. The peculiar, subtriangular hind wing shape, highly simplified venation with the loss of crossveins and braces, and principal veins ending before reaching the posterior wing margin are autapomorphies of the order.

The order has had a complex history (Kinzelbach, 1971, 1990; Kristensen, 1995, 1999; Lawrence \& Newton, 1995; Lawrence et al., 1995; Whiting et al., 1997) and relationships are still in dispute, due in great part to their parasitism and small size. At present, there are only two hypotheses which have any support: Strepsiptera + Coleoptera (Coleopterida) and Strepsiptera + Diptera (Halteria). According to most recent reviews (Whiting \& Wheeler, 1994; Whiting et al., 1997; Whiting, 1998; Wheeler et al., 2001; Whiting, 2002) the Halteria hypothesis is strongly supported by both morphological and molecular data, while the only synapomorphy uniting Coleoptera and Strepsiptera is "flight by posteromotorism"; all other data is said to be based on an "erroneous interpretation of strepsipteran morphology". In their self-righteous criticism of Kukalová-Peck \& Lawrence (1993) by Whiting \& Kathirithamby (1995) it is stated that our evidence for Coleoptera + Strepsiptera was based on "methodological improprieties" and "observational discrepancies". It is true that our case for monophyly was not supported by clearly defined synapomorphies, nor was our data subjected to a modern (numerical) cladistic analysis; we hope this has been remedied in the present paper. As for the use of groundplans rather than exemplars in a phylogenetic study of the higher taxa, we would like to think that varied approaches to reconstructing phylogenetic trees have not yet been narrowed to one appropriate and "foolproof" formula that everyone must follow. As for the so-called errors in observation, at least some of these reflect our critics' lack of understanding of wing morphology. As an example, the authors claimed that Strepsiptera wings start with a convex subcosta, but this vein is always concave and cannot replace the costa on the anterior margin. Instead, strepsipteran wings start with a peculiar, broad, convex anterior margin and the concave subcosta is weak and hidden in an adjacent groove. A similar (very probably synapomorphic) condition occurs in Coleoptera, in which the subcosta sometimes sinks so deeply between the anterior margin and convex radius (RA) that $\mathrm{ScP}$ can be seen only on the ventral side of the wing. In short, the taxon Halteria (Strepsiptera + Diptera) is completely unsupported by wing morphology. The unreduced wing pair in Diptera is the fore wings and in Strepsiptera the hind wings, and the venation and articulation is unrelated (see Figs 28-30, 33). Neoptera have heteronomous wing pairs and fore wings are readily distinguished from hind wings by their veinal arrangement (here on p. 106 in "Neoptera: basal divergence in wing structure"; see also captions to Figs 28-32, and Character 13, below). Kukalová-Peck's (1997) rebuttal of the Whiting \& Kathirithamby paper was considered inconclusive by Kristensen (1999), who apparently misunderstood two of her comments: the first concerning the coleopteran $3 \mathrm{Ax}$ with a separate axillary muscle disc, which shows important similarities to Strepsiptera but is different from all other Neoptera, and the other pointing to the radial arrangement of the wing venation in Strepsiptera. Radially arranged veins occur exclusively in the hind wings in Neoptera and show that the much analyzed "Halteria hypothesis" suggesting a switch between the fore and hind wing pairs in Strepsiptera, is morphologically impossible.

In the best tree of a recent analysis by Beutel \& Gorb (2001), the Coleoptera + Strepsiptera clade was supported by three synapomorphies (excluding those involved with posteromotorism), while 10 additional (less parsimonious) steps were required to support an Antliophora + Strepsiptera clade and 11 to support a Diptera + Strepsiptera clade.

Coleoptera (Figs 19-27, 31, 34L, 35L). This order is exceptional in combining a unique mixture of highly apomorphic characters built around a deeply plesiomorphic venation. The fore wings heightened their protective function by becoming heavily sclerotized, more or less convex, shortened and flush with the body, while retaining the full protowing number of veinal branches. The hind wings increased dramatically in size, while the venation changed almost beyond recognition to fold and fit under the elytra. Only Strepsiptera share the "shrinking" of the fore wing, the enormous enlargement of the hind wing, the switch to postero-motoric flight, and the corresponding unique fusions, reductions, fragmentation and membranization in their pteralia. The plesiomorphic radius, also present in Strepsiptera (Figs 28-30), shows the sectors RA and RP basally not connected or barely touching. A similar absence of the stem of $\mathrm{R}$ is known only in the Paleozoic (!) pleconeopterans and hemineopterans (Sharov, 1966; Kukalová-Peck \& Brauckmann, 1990, Figs 32, 33) and in the prothoracic "protowings" of Palaeodictyoptera and Odonatoptera: Geroptera (Fig. 1A, B) (Wootton \& Kukalová-Peck, 2000; Haas \& Kukalová-Peck, 2001, introduction to Table 6). Except for the close relationship to Strepsiptera, the wing characters of Coleoptera are so changed by elytral formation and apical folding that similarities with other endoneopteran groups are difficult to find and are not very convincing. Autapomorphies include: two reinforced wing struts (radial and medial bars) connected basally by a bridge; struts ending distally with apically 
attached, collapsing veinal loops; bending zones on each of the wing struts present; RP branches flattened and arched in the apical field; veins flanked by broad sclerotized strips; extra-strong and long cross-vein braces; and veinal cells acting as plates. The absence of the stem of $\mathrm{R}$ in fossil Hemineoptera and basal Coleopterida indicates that the stem of $\mathbf{R}$ was probably absent in the ancestor of Hemineoptera + Endoneoptera, and that Coleoptera, like Hymenoptera, may have diverged from the common endoneopteran stock very early.

\section{Characters and character state distribution}

All characters were considered to be polarized and unordered.

0. Flight mechanism: 0, with hind wings dominant; 1, with fore wings dominant; $\mathbf{2}$, with fore wings shortened, not capable of flight, and hind wings the only flying pair. In Orthoneoptera and Blattoneoptera, both pairs of wings participate in flying but the fore wings are narrower, more or less tegminous and protective, while the hind wings are broader, thinner, and a more powerful flying pair. In Hemineoptera and Endoneoptera, fore wings become larger and usually also thinner, and serve as the principal flying pair, while the hind wings are reduced in size and their veinal support is diminished. Note that in Coleoptera and Strepsiptera, this trend was suppressed. Exceptionally, Fulgoromorpha, Heteroptera, Trichoptera and Lepidoptera may develop secondarily enlarged hind wings, but stronger flight musculature occurs in the mesothorax.

1. Hind wing apex: 0 , well behind anterior edge of wing base (at same level as cubital basivenale); 1, at same level as anterior edge of wing base, $\mathbf{2}$, well in front of anterior edge of wing base. In all outgroups and in Coleoptera, Neuropterida and Mecopterida (Figs 5-22), the hind wing apex is positioned well behind the anterior wing margin at about the same level as the cubital basivenale. In Hymenoptera, the apex has shifted anteriorly, so that it lies at almost the same level as the anterior edge of the wing base; the anterior wing margin is more or less perpendicular to the long axis of the body and is hooked to the dominant fore wing. In Strepsiptera (Figs 28), the apex is in front of the wing base; the anterior wing margin is oblique and is not attached to the highly reduced fore wing.

2. Wing flight units: $\mathbf{0}$, separated by claval flexion line; $\mathbf{1}$, separated by anal fold. In Orthoneoptera (Fig. 2), the leading flight unit (i.e. the remigium) is separated from the trailing flight unit (i.e. the anojugal lobe) by a claval flexion line. In Blattoneoptera, Hemineoptera and Endoneoptera (Figs 2, 10, 15, 18, $23,28)$, the leading flight unit becomes extended and is separated from the trailing flight unit by the anal fold. This difference promoted further diversification and resulted in different types of flight.

3. Anojugal lobe (veinal support): $\mathbf{0}$, full, including AA; 1 , partial, excluding AA. In Orthoneoptera, the hind wings bear a large, full anojugal lobe supported by all anal $(\mathrm{AA} 1+2, \mathrm{AA} 3+4$, $\mathrm{AP} 1+2, \mathrm{AP} 3+4)$ and jugal (JA, JP) veins (Fig. 2). In Blattoneoptera, Hemineoptera and Endoneoptera (Figs 3, 5, 10, 15, 18, 19, 28 ), the hind wings bear a partial anojugal lobe supported only by posterior anal $(\mathrm{AP} 1+2, \mathrm{AP} 3+4)$ and jugal (JA, JP) veins.

4. Partial anojugal lobe (intercalaries): 0 , lacking intercalary branches; $\mathbf{1}$, with pectinate intercalary branches. Among the lineages with a partial anojugal lobe, Hemineoptera and Endoneoptera (Figs 2, 3, 5, 10, 15, 18, 19, 28) bear a lobe which is supported by primary anal and jugal veins, which are dichotomously branched in the more basal groups and simple or reduced in various derived groups. In Blattoneoptera alone (Fig.
2), this lobe is enlarged and supported not only by branches of $\mathrm{AP}$ and $\mathrm{J}$, but also by numerous intercalary veins in AP area.

5. Anojugal lobe (extent): 0, broader than remigium and extending well beyond mid wing; 1, slightly narrower than remigium and ending at or slightly beyond mid wing; 2 , much narrower than remigium and ending at about mid wing; $\mathbf{3}$, much narrower than remigium and ending between basal third and midwing; 4, much narrower than remigium and ending before basal third. In Orthoneoptera (Fig. 2), the full anojugal lobe is broad, occupies most of the hind wing area, and ends close to wing apex. In Blattoneoptera, the partial lobe is much shorter and smaller in basal taxa (as in Paleozoic ancestral blattoneopterans and basal Blattodea) but in some groups it has become secondarily enlarged with a support from intercalary veins: it ends before mid-wing in Isoptera, beyond mid-wing in Mantodea and most Blattodea (Fig. 2), and at the wing apex in Dermaptera and the cockroach family Blattellidae, which are capable of apical folding. In Hemineoptera and Endoneoptera, the partial lobe is shorter and progressively narrowed and diminished in both groups. In Hemineoptera (Fig. 2), the lobe usually ends at about mid wing, while in Hymenoptera (Figs 3, 4) it is narrower and ends between basal third and mid wing. In Coleoptera, Strepsiptera, Neuropterida and Mecopterida (Figs 5-28), the lobe ends before the basal third, but in Coleoptera (Figs 23-26), it becomes secondarily broadened. Note that in some derived Heteroptera, Fulgoromorpha, Trichoptera and Lepidoptera, the anojugal lobe may become secondarily broadened.

6. Anojugal lobe (posterior limits): 0, protruding posteriorly beyond level of remigium; $\mathbf{1}$, aligned with or narrower than remigium. In Orthoneoptera, Blattoneoptera, Hemineoptera (Fig. 2) and Hymenoptera (Figs 3, 4), the anojugal lobe is protruding posteriorly well beyond the posterior margin of the remigium. In Coleoptera and Strepsiptera (Figs 23-26, 28), this lobe is both narrower and aligned with the remigium (note that in the latter group, the posteriorly protruding portion of the wing is occupied by a small anojugal lobe and also by the enlarged proximal end of the remigium). In Neuropterida and Mecopterida (Figs 5-14), the anojugal lobe is significantly narrower than the remigium, but may have been secondarily broadened in large sized Megaloptera (Fig. 7).

7. Anojugal lobe in hindwing: 0, larger than in fore wing; 1, smaller than in fore wing. In non-Endoneoptera and in Hymenoptera, the anojugal lobe in the hind wing is larger than in fore wing. In Neuropterida (except in Megaloptera, Fig. 7) and Mecopterida (except in some derived Trichoptera), the anojugal lobe is shorter and narrower than in fore wing. In Megaloptera and Trichoptera, the broad lobe appears to be secondary since supporting veins are simplified, as they usually are in smaller wings.

8. Claval flexion line (expression): 0 , complete to wing margin, separating remigium from full anojugal lobe; $\mathbf{1}$, incomplete or absent, not separating remigium from anojugal lobe. In Orthoneoptera (Fig. 2), the claval fold functions in flight as a full-length flexion line between the remigium and the full anojugal lobe. In most Blattoneoptera, Hemineoptera and Endoneoptera (Figs 2, 3-5, 10, 15), this flexion line is incomplete, often weakened and non-functional or absent. In the hind wings of some Mantodea, large Fulgoromorpha (Fig. 2) and Hymenoptera (Fig. 3, 4), this line may be deeply impressed proximally, but it flattens out near the wing margin and does not form a complete separation between the remigium and anojugal lobe.

9. Claval flexion line in Endoneoptera: 0, deep and curved; 1, shallow and not curved; 2, absent. In Hymenoptera (Figs 3, 4) (and also Fulgoromorpha, Fig. 2), the claval flexion line no 


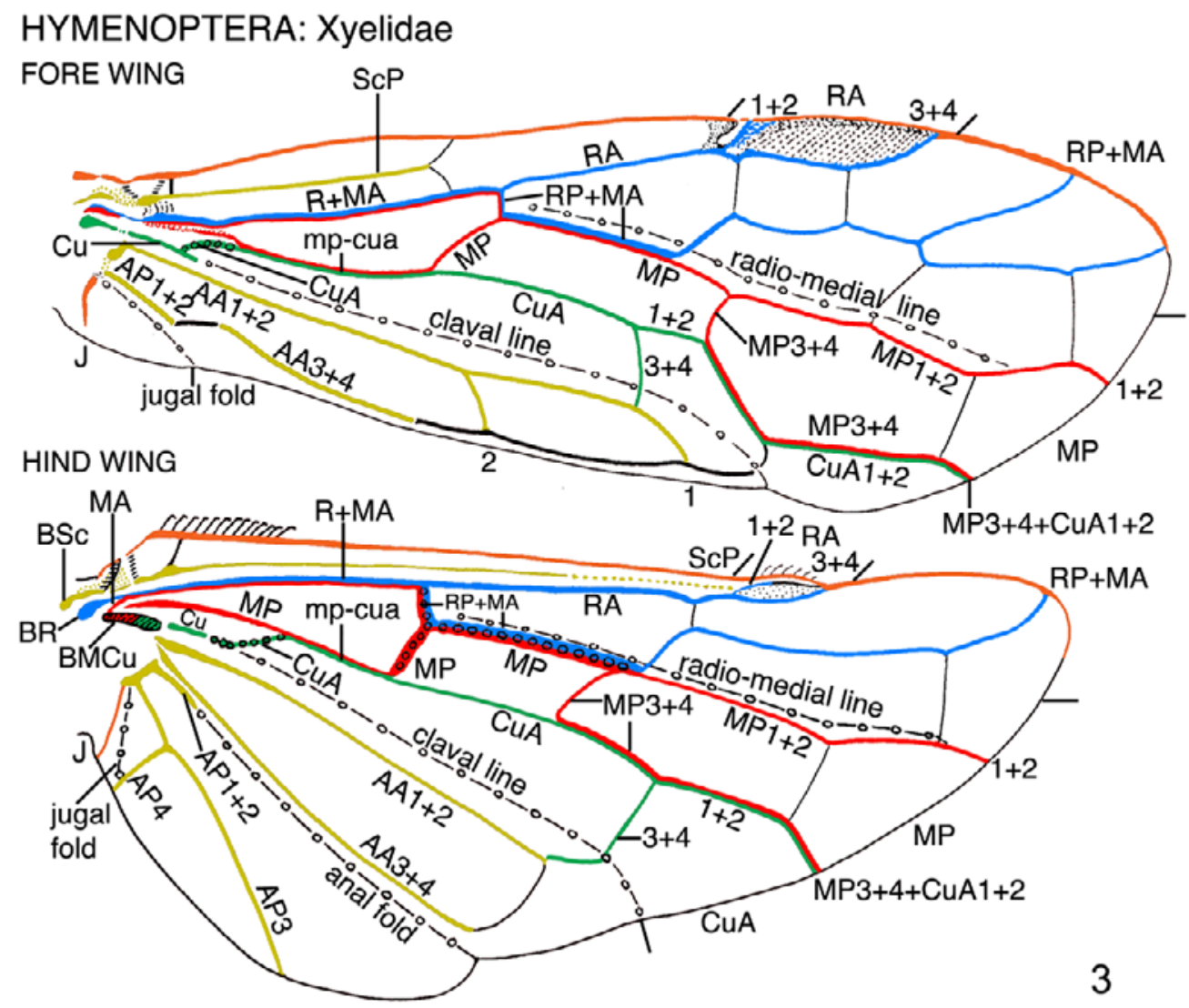

HYMENOPTERA: Xyelidae

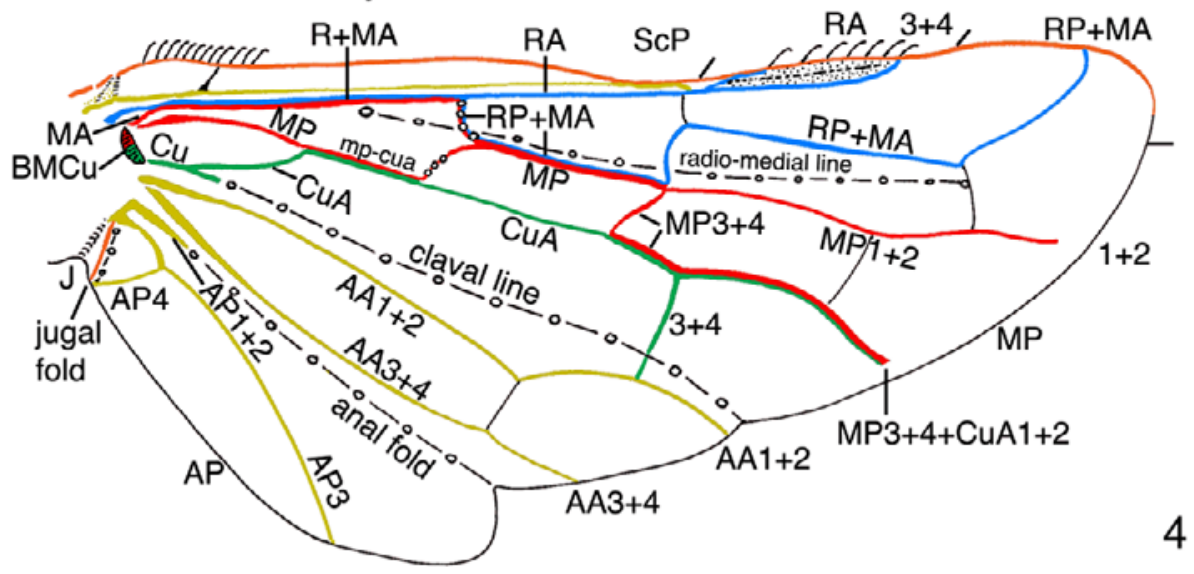

Figs 3-4. Hymenoptera, forewing and hindwing. In these and all following figures, the color scheme for veinal systems and aligned rows of pteralia is as follows: costal, orange; subcostal, yellow; radial, blue; medial, red; cubital, green; anal, yellow; jugal, orange. Hymenoptera: Xyelidae. In the hind wing, a highly derived pattern of extensive veinal reductions, hyaline membrane, enhanced sparse cross-vein braces and a complex, partly membranized media with three long fusions, is uniquely combined with a plesiomorphic broad anojugal lobe, long prominent claval flexion line and anal fold, broad AA area and retention of AA1 +2 , and sets the group far apart from the rest of Endoneoptera. Open beads mark membranized portions of veins, including a unique, long, "Y"-shaped fusion brace centrally in the hind wing. 3 - Xyela nearctica (Ross, 1932), fore and hind wing compared; 4 - Pleroneura bruneicornis Rohwer, 1910 (a hymenopteran hind wing with the least reduced venation).

longer marks the beginning of the anojugal lobe, but is still deep and curved. In Neuropterida (Figs 5-9) and Mecopterida (Figs $10-14)$, it is shallower, more or less straight, and often significantly shortened. In Coleoptera and Strepsiptera (Figs 23-26, $28)$, it is absent.
10. Anal fold (expression): 0 , same as other folds in anojugal lobe; $\mathbf{1}$, highly convex, long and distinct; $\mathbf{2}$, shortened or absent. In Orthoneoptera (Fig. 2), the anal fold is not different from other folds in the full, fan-like anojugal lobe. In Blattoneoptera (Fig. 2), it is highly convex and prominent (in fossil blattoneop- 
NEUROPTERA: Ithonidae
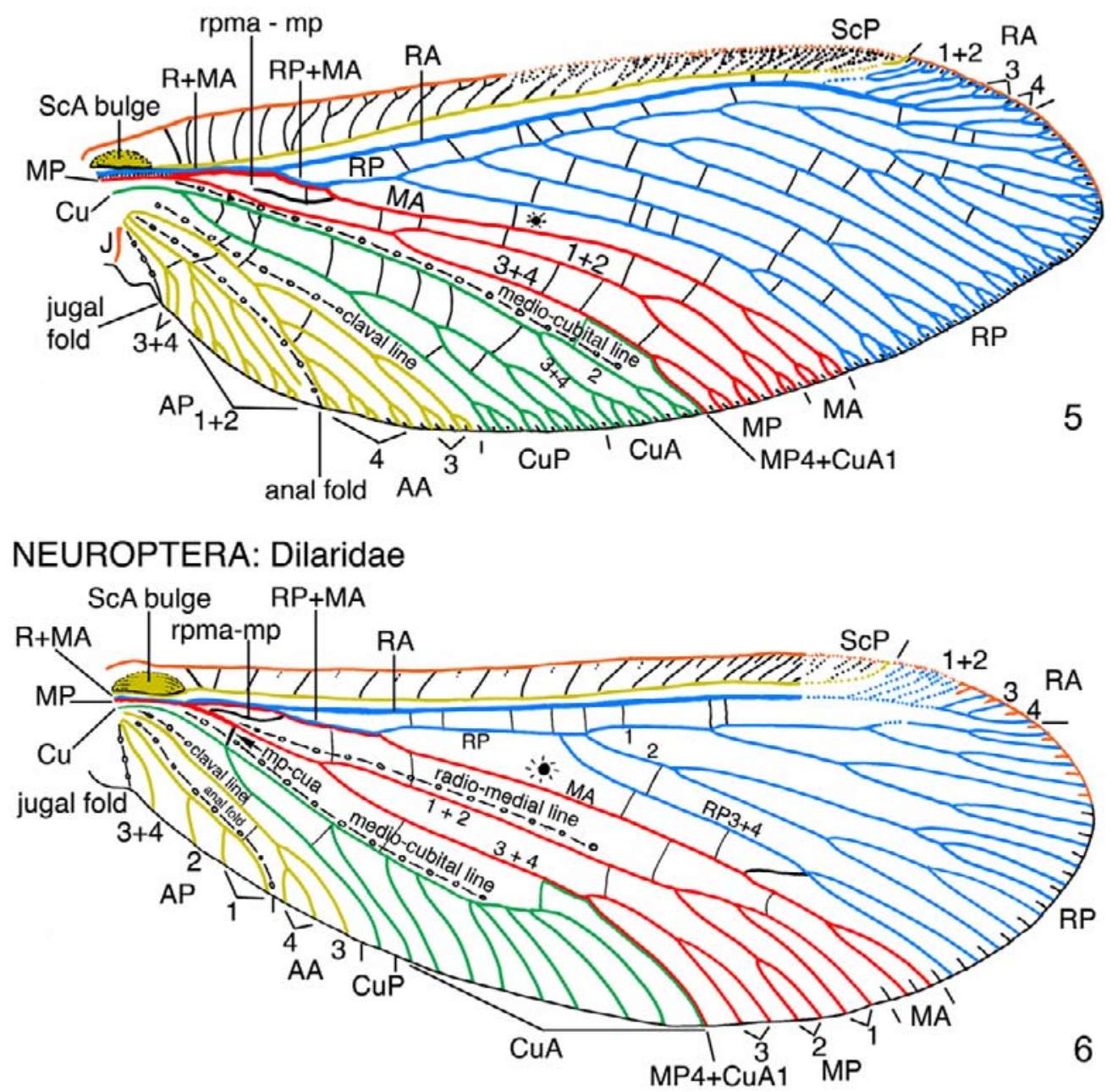

Figs 5-6. Neuroptera, hind wing. 5 - Ithonidae: Ithone sp. Softened part of the anterior margin dotted; the stem of R present, short; MA fused with R immediately at base; RA \& RP long, closely parallel, connected by backwards slanted ra-rp cross-veins; RP branches long, parallel, densely arranged; brace rpma-mp long, sinusoid (homologous to medial bridge in Coleoptera and bar-like brace in Diptera, Figs 31,33) (six neuropterid apomorphies); MP abuts basally with R + MA but keeps its own trachea; MA early and visibly separates from RP; fusion MP4 + CuA1 short, plesiomorphic (found also in basal Coleoptera in Polyphaga and Archostemata); nygma anterior to MA retained; anal fold long (five plesiomorphies). The base of RP near its divergence from MA often reduced and replaced by a slanted ra-rp cross-vein; AA1+2 lost; short intercalary branches along the wing margin added (three Neuroptera apomorphies); 6 - Dilaridae: Dilarinae: Nallachius sp. As in Ithonidae but RP branches fewer, MA separates later, CuA enriched by pectinate intercalary branches; CuP simple; anojugal lobe much more diminished, very short and narrow (five apomorphies).

terans and Isoptera, the anojugal lobes fold at rest under remigium like a book along the convex anal fold). In Hemineoptera, Hymenoptera, Coleoptera and Strepsiptera (Figs 2-4, $23-26,28)$, the fold is convex and prominent, although it is crossed and interrupted by AP in the first group. In Neuropterida (Figs 5-9) it is weakly expressed and shortened or sometimes absent (in Raphidioptera), while in Mecopterida (Figs $10-14)$ it is always absent. Note that in the fully homologized scheme, anal fold starts always basally between AA $3+4$ and AP, and it should not be confused with other nearby folds, such as those between $\mathrm{AA} 1$ and $\mathrm{AA} 2$ or $\mathrm{AA} 2$ and $\mathrm{AA} 3+4$, which are pronounced in some groups (e.g., Plecoptera).

11. Anal fold: $\mathbf{0}$, not crossing AP1+2; $\mathbf{1}$, crossing AP1+2. In Orthoneoptera, Blattoneoptera and Endoneoptera, the anal fold lies in membrane between $\mathrm{AA} 3+4$ and $\mathrm{AP} 1+2$. In Hemineoptera, the fold crosses the first branch of AP (Fig. 1).
12. Tegula: 0, present; 1, absent. In Orthoneoptera, Blattoneoptera, Hemineoptera, Hymenoptera, Neuropterida and Mecopterida, the tegula is present. In Coleoptera and Strepsiptera (Figs 29-31), it is absent in both wing pairs. Note that in Strepsiptera the large bulbous sclerite, aligned with the anterior wing margin and occurring near the tergum, is not homologous with the tegula, but with the precosto-costal proxalare (PRC) of the fully homologized neopteran scheme (Haas \& KukalováPeck, 2001, Figs 1A, B, C). The same plesiomorphic PRC sclerite occurs also in Coleoptera (Kukalová-Peck \& Lawrence, 1993, Fig. 78), Neuropterida, Mecopterida and elsewhere, but is very small.

13. Anterior margin near wing base: 0 , evenly sclerotized; 1, with desclerotized area. In dry specimens of neopteran hind wings, the anterior margin near the base is well sclerotized and lying flat. In Coleoptera, a portion close to base is pliable due to 
MEGALOPTERA: Corydalidae

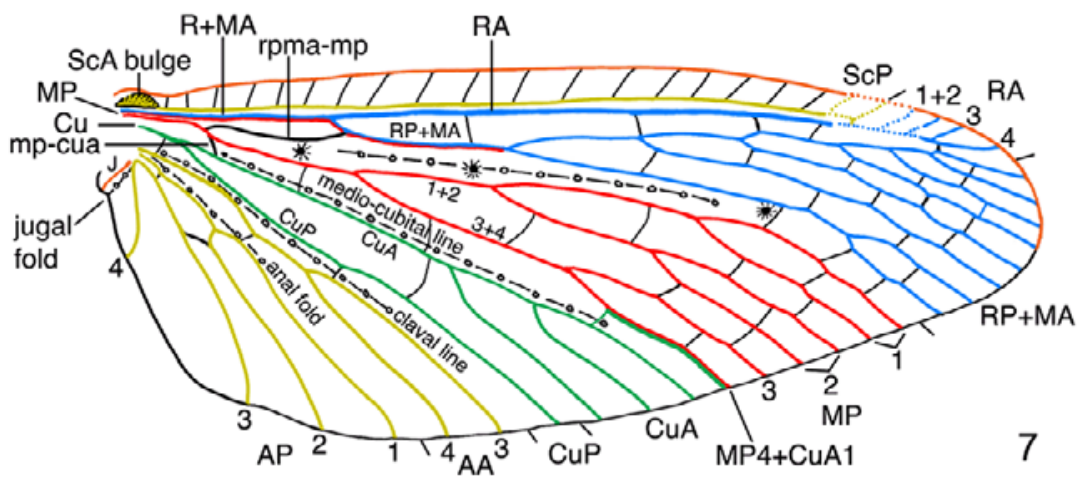

MEGALOPTERA: Corydalidae

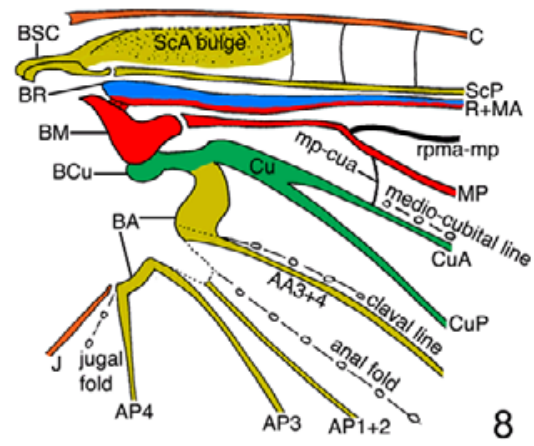

Figs 7-8. Megaloptera, hind wing: Corydalidae: Neohermes sp., hindwing and base of the hind wing. Veinal characters as in Neuroptera, but R longer; RP area smaller; veinal branching impoverished; MA apically not discernible from RP branches; CuA less branched, CuP simple; nygmata placed posterad to MA (or RP + MA); claval and anal fold shallow, shortened (anal fold more derived than in Coleoptera); and AP branches simplified but anojugal lobe relatively very broad (probably secondarily enlarged) (all apomorphies).

the humeral fold, and it often rolls up and backwards, concealing ScP at the bottom of a parallel groove. A similar, unusual rolling condition occurs in the anterior margin of Strepsiptera. This was illustrated by Whiting and Kathirithamby (1995), but these authors erroneously interpreted the convex anterior wing margin as a convex "vein" ScP. This is not possible because in the homologized protowing and all Pterygota, the anterior margin is always convex and ScP concave (-).

14. Anterior margin near wing apex: 0 , entirely sclerotized; $\mathbf{1}$, with a desclerotized zone. In non-Neuropterida, the distal portion of the anterior wing margin is reinforced by sclerotization. In Neuroptera and Megaloptera, there is a large anterior portion of the wing apex, including the anterior margin, the end of ScP and the branches of RA1+2, which are desclerotized and quite pliable. In Raphidioptera, the weakness in the anterior margin is secondarily strengthened by support from the arched end of ScP (Fig. 9).

15. ScA: 0, a branched vein, not modified; 1 , forming a short, blunt ridge; $\mathbf{2}$, reduced; $\mathbf{3}$, forming a bulge. In Orthoneoptera (and ancestral fossil Pleconeoptera), $\mathrm{ScA}$ is a relatively long and dichotomously branched vein (Fig. 2). In Blattoneoptera, it forms a narrow, oblique and blunted ridge. In Hemineoptera, $\mathrm{ScP}$ dives under $\mathrm{R}$ and $\mathrm{ScA}$ is reduced. In Endoneoptera, the $\mathrm{ScA}$ ridge is expanded into a sclerotized bulge.

16. ScA bulge: 0 , not broad, convex and heavily sclerotized; 1, broad, convex and heavily sclerotized. In Hymenoptera, ScA bulge is relatively narrow, low, and unevenly sclerotized. In Coleoptera, Strepsiptera, Neuropterida and Mecopterida, the ScA bulge is broad, convex, and moderately to heavily sclerotized (Figs 5-15, 29-31). Note that the bulge becomes secondarily oblique and narrow in the petiolate wings of derived Mecoptera and Diptera (Figs 14, 33).

17. ScA bulge: 0 , not distally delimited by fold; 1 , distally delimited by deep fold. In Hymenoptera (Figs 3, 4), the ScA bulge is separated distally from the rest of wing by a deep fold that also bisects ScP.

18. ScP: 0, ending well before RA forks into RA1+2 and RA3 +4 ; 1, ending beyond RA fork and distally overlapping RA1+2. In Orthoneoptera, Blattoneoptera, Hemineoptera (Fig. 2), Hymenoptera, Neuropterida and Mecopterida (Figs 3-18),
$\mathrm{ScP}$ ends before RA, forks into RA1+2 and RA3+4, and the RA branches end close to the wing apex. In Coleoptera and in some Strepsiptera (Mengenilla, Coriophagus, Figs 19-22, 28), ScP is longer than the beginning of RA1+2, so that they distally overlap. In Coleoptera, this overlap is exploited in apical folding when ScP fuses with an equally long RA $+\mathrm{RA} 1+2$ to form an anterior strut called the radial bar by Kukalová-Peck \& Lawrence (1993).

19. BSc, BR and base of ScA: 0, not fused into plate; 1, fused to form heavily sclerotized plate. In all the outgroups and in most Endoneoptera, the subcostal basivenale (BSc), the radial basivenale (BR) and the basal portion of $\mathrm{ScA}(\mathrm{ScA}$ bulge) are individually delimited, more or less separated by articulations and furrows, and never fused into a single large plate. In Coleoptera and Strepsiptera (Figs 29-31), BSc and BR are fully fused together and form with the inflated, enlarged ScA bulge a prominent, heavily sclerotized plate, which in beetles is involved in the folding process. This combined plate also includes the anterior margin and humeral plate in most Coleoptera and in all Strepsiptera.

20. Pterostigma: 0, absent or not supported by reinforced cross-veins; 1, supported by reinforced cross-veins. The pterostigma is a blood sinus and pump occurring in many Neoptera and Paleoptera and possibly plesiomorphic for Pterygota. It is usually darkly pigmented and lies between two branches of RA, as in Raphidioptera (Fig. 9) and Strepsiptera (Fig. 28), and in Polyphaga (Fig. 19), where the branches unite again to form the radial cell. Support of the pterostigmal area by reinforced radial cross-veins (state 1) occurs in Archostemata, Myxophaga and Adephaga.

21. Radial cross-veins: $\mathbf{0}$, serial and not reinforced; 1 , forming braces, r1, r2, r3, r4; 2, absent. In basal Orthoneoptera, Blattoneoptera, Hemineoptera, Hymenoptera, Neuropterida and Mecopterida, ra-rp cross-veins are weak, short and serially homonomous. In Coleoptera (Figs 19-22), they form elongate and reinforced radial cross-veins $\mathrm{r} 1, \mathrm{r} 2, \mathrm{r} 3$ and $\mathrm{r} 4$, which provide important braces in apical folding. In Strepsiptera, all crossveins are absent.

22. Reinforced cross-vein $\mathbf{r} 1$ in Coleoptera: 0, present; 1 , absent. Specialized radial cross-vein braces are absent in all 


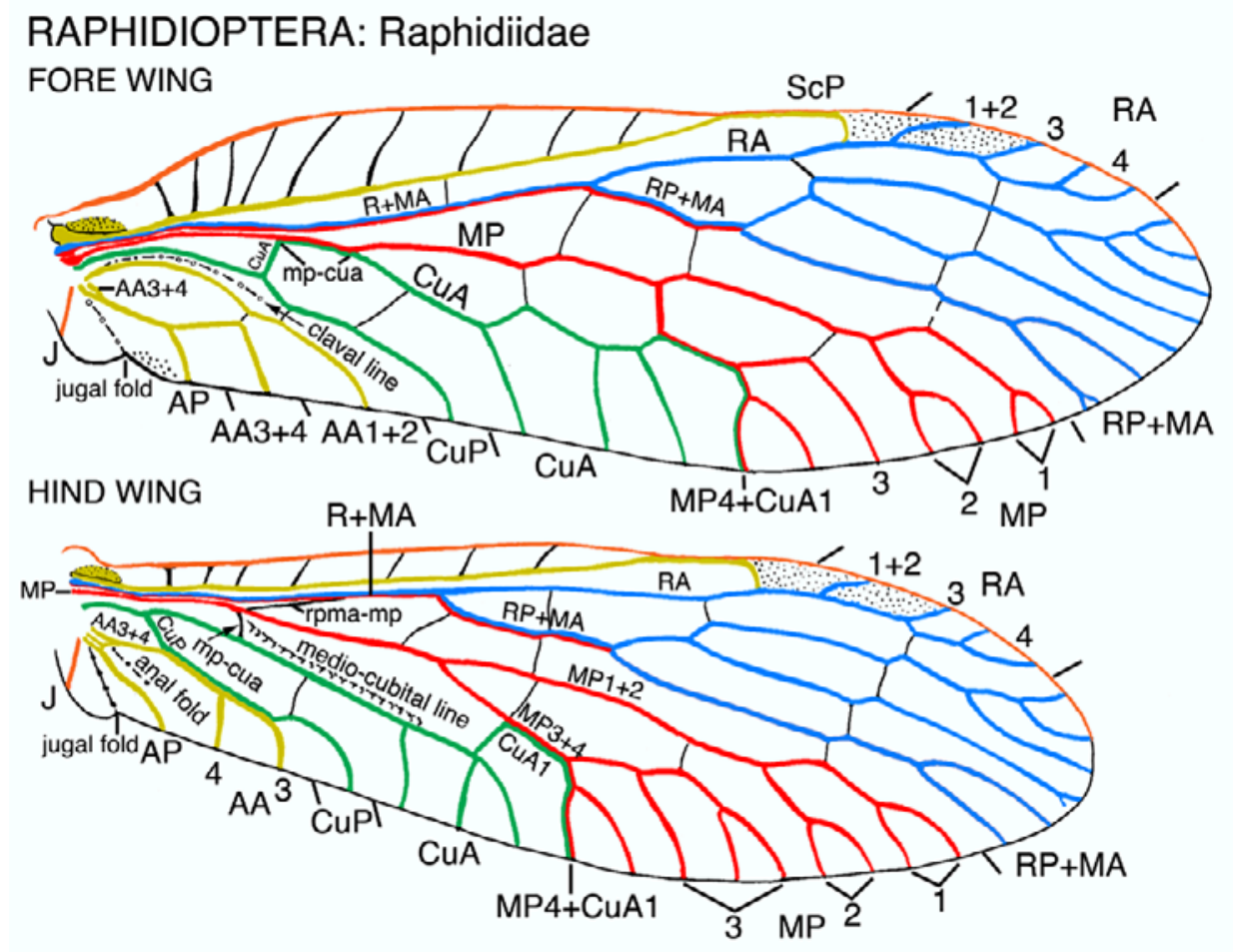

Fig. 9. Raphidioptera, fore and hind wing. Raphidiidae: Agulla (Glavia) adnixa (Hagen, 1861). Veinal characters as in Megaloptera, but hind wing narrowed basally; wing membrane hyaline; cross-veins strong, few, causing veins to angle; ScP arched apically, solidifying the anterior margin; the stem of R significantly longer; $\mathrm{CuP}+\mathrm{AA} 3+4$ abutment well developed; anal branches short and very few. Note that rpma-mp cross-vein brace, which is homologous with the medial bridge in Coleoptera, shortly annexed veins near the base in Mecoptera, and the prominent straight cross-vein brace in Diptera (a brace synapomorphy of Coleopterida + Neuropterida + Mecopterida), was misinterpreted as a principal vein by Willmann (1994).

groups except Coleoptera (see also next character). The first radial cross-vein, $\mathrm{rl}$, is well developed in Archostemata, Adephaga and fully winged Torridincolidae (Myxophaga). It is absent in all Polyphaga and the myxophagan genera Lepicerus, Sphaerius and Hydroscapha.

23. Reinforced cross-vein $\mathbf{r} 3$ in Coleoptera: 0, present; 1 , highly reduced or absent. The third reinforced ra-rp cross-vein, r3, is present in Archostemata and Polyphaga. In the latter group it is often longitudinally oriented and has been mistaken for a vein; note that the $\mathrm{r} 3$ cross-vein is independently lost in various derived polyphagan taxa. The absence of cross-vein $r 3$ is characteristic of Adephaga and Myxophaga (Figs 20-22). The only exception in Adephaga is the apparent stub of $\mathrm{r} 3$ shown in Macrogyrus (Kukalová-Peck \& Lawrence, 1993, Fig. 16), but absent in Spanglerogyrus.

24. Radial cell: $\mathbf{0}$, absent, RA $1+2$ and $\mathrm{RA} 3+4$ diverging only; 1, present, formed by divergence and convergence of RA1+2 and RA $3+4$. The branches RA $1+2$ and RA $3+4$ diverge and end independently on anterior margin in non-Coleoptera, including Strepsiptera (Fig. 28), and also in Archostemata (Fig. 20), Adephaga (Fig. 21) and Myxophaga (Fig. 22). The radial cell, formed by the reuniting of $\mathrm{RA} 1+2$ and $\mathrm{RA} 3+4$, is unique to Polyphaga (Fig. 19).

25. RA forking: 0, close to wing apex; 1 , close to mid wing. In Orthoneoptera, Blattoneoptera, Hemineoptera, Hymenoptera, Neuropterida, and Mecopterida, RA forks to form the branches $\mathrm{RA} 1+2$ and RA3+4 well beyond the mid wing and relatively close to the wing apex. In Strepsiptera (Fig. 28) and Coleoptera (Figs 19-22), RA forks close to the mid wing.
26. RA3+4 in Coleoptera: 0, entire; 1 , membranized basally. State 1 is unique to Myxophaga (Fig. 22).

27. RA3-RA4 fork in Coleoptera: 0, close to anterior margin; 1, far from anterior margin. In most Neoptera, the divergence of RA3 and RA4 occurs close to the anterior margin. This fork occurs well behind the anterior margin in Archostemata, Myxophaga and Adephaga (Figs 20-22).

28. RA3+4 and RA3 in Coleoptera: 0 , not forming right angle: 1, forming right angle. In Polyphaga, Archostemata and Myxophaga (Figs 19, 20, 22) the base of RA3 is almost at the same level as the distal end of RA3+4. In Adephaga (Fig. 21), RA3 diverges from RA3+4 at a right angle or close to it.

29. RA3: 0, short or absent; 1, long, parallel to anterior margin; 2, reduced to short stump. In non-Coleoptera, RA3 is short and oblique and absent when RA3+4 is not subdivided. In Polyphaga, Myxophaga and Adephaga (Figs 19, 21, 22), RA3 is much longer and runs parallel to the anterior wing margin. Reduction to a short stump occurs only in Archostemata (Fig. 20).

30. RA4 (fusion with RP1): $\mathbf{0}$, short and oblique or absent; 1, long, parallel to anterior margin and independent; 2, long, parallel to anterior margin and fused at length with RP1. In nonColeoptera, RA4 is short and obliquely oriented, and absent when RA3+4 is not subdivided. In Archostemata, Myxophaga and Adephaga (Figs 20-22), RA4 is long, almost parallel with the anterior margin, and independent. In Polyphaga (Fig. 19), it is fused at length with RP1. Although the fusion appears to be a basal polyphagan feature, RA4 is lost in the majority of polyphagan families. 

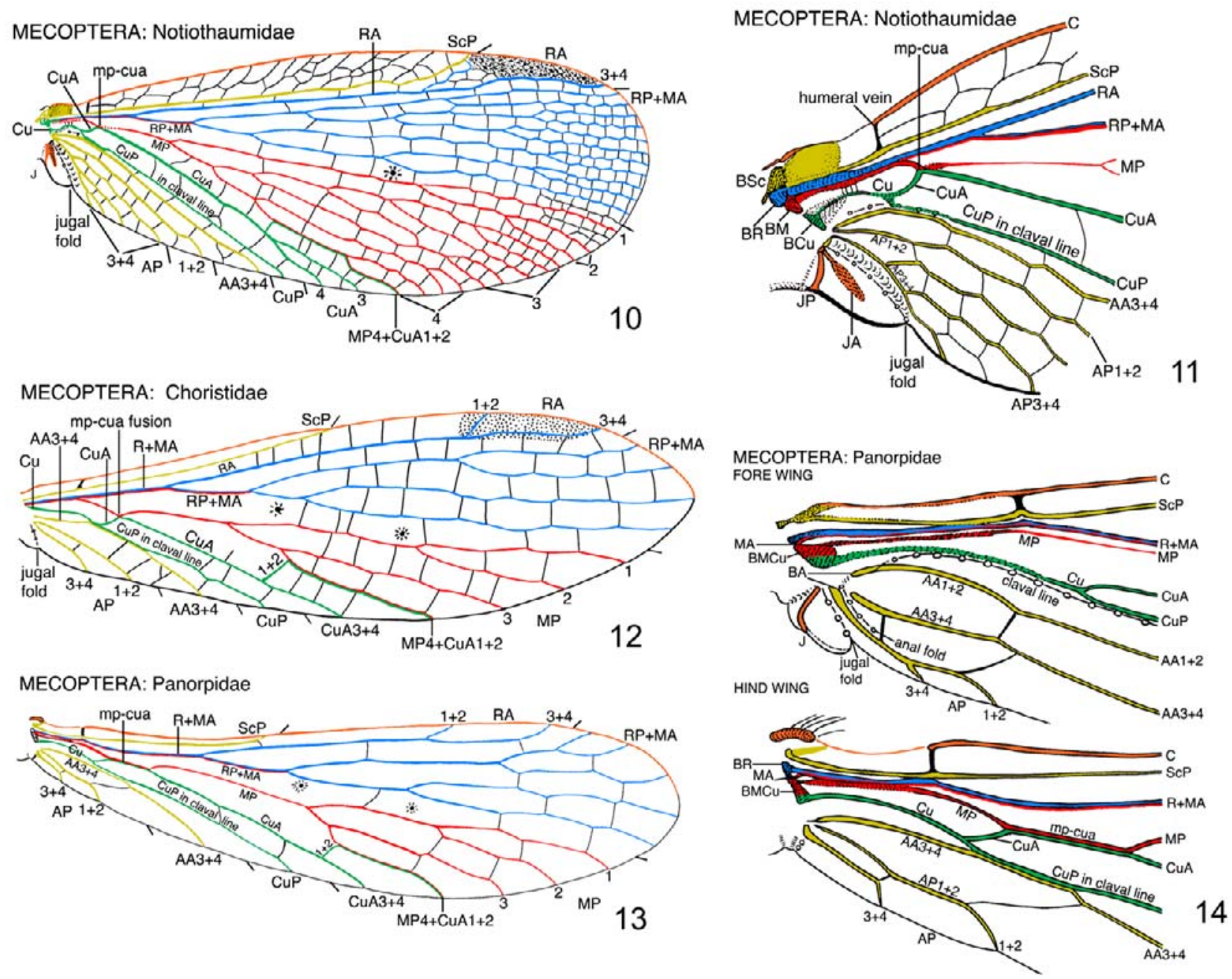

MECOPTERA: Panorpidae

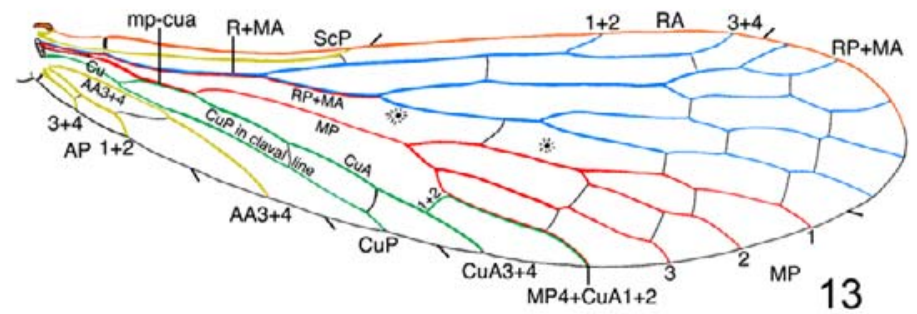

Figs 10-14. Mecoptera, fore and hind wing. Veinal features similar to Neuropterida in RA and RP area, but closer to base extensively obscured by petiolation; anterior margin solidified; ScP tends to shorten; MA not discernible apically from RP branches; rma-mp brace (replacing the rpma-mp brace because of a longer stem of R and consisting of MP abutting R + MA) is extended by a membranous strip between MP and Cu (Fig. 11, four parallel lines); nygmata placed posterad of RP + MA; mp-cua brace varies from point fusion to elongate fusion; the section of MP beyond mp-cua brace weakened; anal fold lost; fusion MP $4+\mathrm{CuA} 1+2$ longer and more derived; $\mathrm{AA} 3+4$ abutting $\mathrm{CuP}$ for a short distance; $\mathrm{CuP}$ runs in a long, shallow claval flexion line; anojugal lobe strongly diminished, short and narrow (all apomorphies). In the fore wing (Fig. 14), MP basally is visibly separate from R + MA (a significant plesiomorphy: separate bases of MA and MP in Endoneoptera are usually visible only in dissections, hence media has been misunderstood). 10-11 - Notiothaumidae: Notiothauma sp., hind wing and base of the hind wing. Characters strongly reminiscent of basal Neuropterida (Ithone): relatively long ScP with plesiomorphic branches; RA \& RP narrowly parallel, connected by slanted cross-veins; RP area very long, filled with densely parallel, long, richly divided branches. Petiolation very slight; mp-cua brace is a point fusion (two plesiomorphies at the Mecoptera level). Fusion MP4 + CuA1+2 obscured by cross-veins; intercalar branches added (two apomorphies); 12 - Choristidae: Taeniochorista sp., hind wing narrow, slightly petiolate, branching simplified; many cross-veins lost; fusion MP4 + CuA1+2 somewhat obscured by cross-veins (all apomorphies); 13-14 - Panorpidae: Panorpa maculosa Hagen, 1861, hind wing and base of the fore- and hind wing. Wings distinctly petiolate; cross-veins further reduced; MP basally free in fore wing but abutted with $\mathrm{R}+\mathrm{MA}$ in hind wing; mp-cua brace is an elongate fusion; fusion MP $4+\mathrm{CuA} 1+2$ distinct (all apomorphies).

31. RA3 and RA4: 0, diverging dichotomously; 1 , forming a right angle. In Polyphaga (Fig. 19), RA3 and RA4 diverge in a regular dichotomous fork. In Archostemata (Fig. 20), the RA3-RA4 divergence forms a very short right angle. In Adephaga and Myxophaga (Figs 21, 22), this angle becomes long and conspicuous.

32. RP branches: 0 , multiple, almost parallel; 1 , reduced to single branch; 2, close basally, radiating apically and usually curved. In Orthoneoptera, Blattoneoptera, Hemineoptera, Neuropterida and Mecopterida, RP branches are repeatedly branched and almost straight. In Hymenoptera (Figs 3, 4), a single RP branch remains. In Coleoptera (Figs 19-22), RP branches are basally close together, curved and apically radiating and shaped like rooster's tail. In Strepsiptera (Fig. 28), the remnants of RP1 and RP2 have a similar pattern (in Mengenilla, Coriophagus and Lychnocolax: Kukalová-Peck \& Lawrence, 1993, Figs 69-71; Kinzelbach, 1971, Fig. 73, A-Z, A'-N').

33. RA and RP: 0, gradually diverging at an angle; 1, running close and parallel for long distance. In most groups, RA and RP diverge gradually at an angle, but in Megaloptera, Neu- 

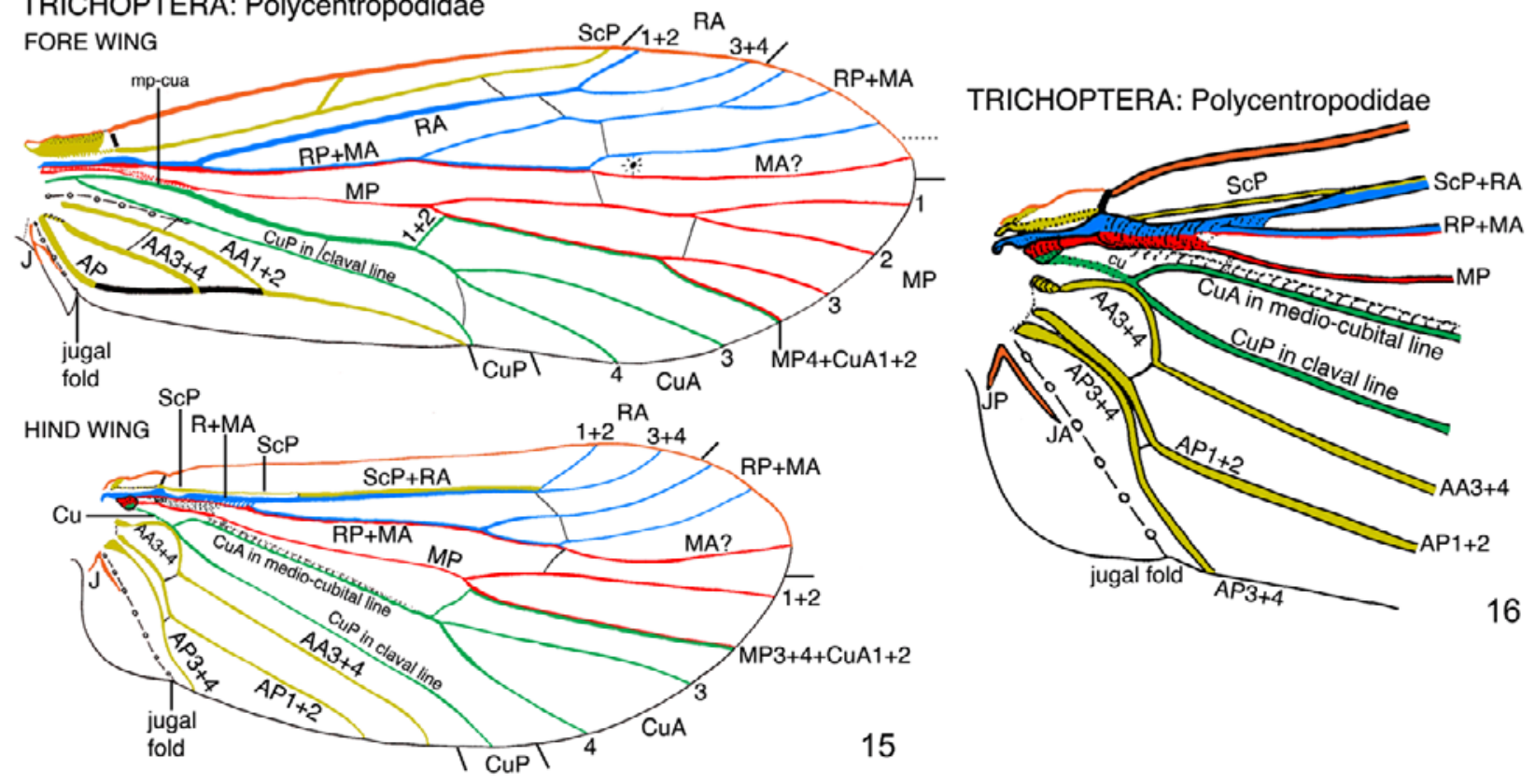

\section{TRICHOPTERA: Philopotamidae} FORE WING

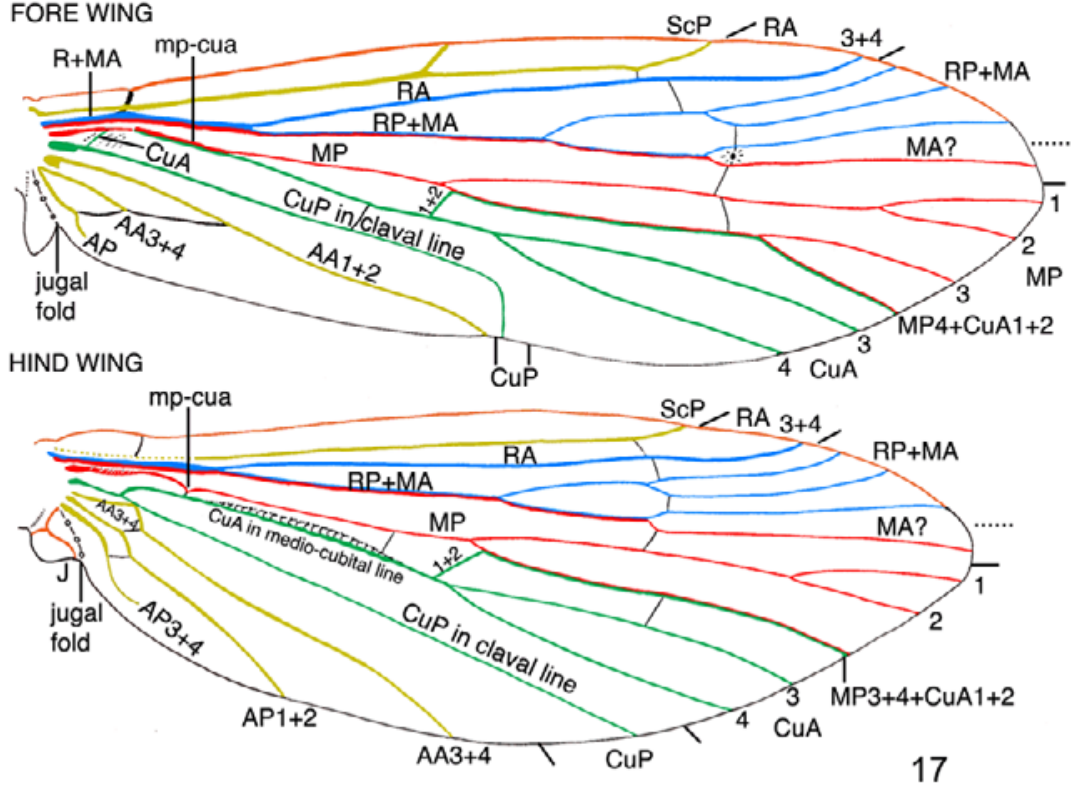

Figs 15-17. Trichoptera, fore wing and hind wing. Veinal features similar to basal Mecoptera but branching much more impoverished; nygmata placed anterior to MA when MA separates from RP but posterior when fused into RP + MA; cross-veins few and weak; fusion brace MP3 $+4+\mathrm{CuA} 1+2$ much longer and more derived, marked by a distinct $\mathrm{CuA} 1+2$ crossing; $\mathrm{CuP}$ runs along bottom of a long, shallow claval furrow (five apomorphies). In the distinctly larger fore wing, veins AA $3+4$ and AP tend to form loops (also occurring in Hemineoptera). Wings not petiolate, claval furrow long (two plesiomorphies). 15-16 - Polycentropodidae: Polycentropus interruptus Ross, 1944, fore- and hind wing, base of the hind wing. In fore wing, MP is basally separate from $\mathrm{R}+\mathrm{MA}$, and mp-cua brace is replaced by MP and $\mathrm{CuA}$ joined shortly by stiffened membrane. In hind wing only, MP abuts basally with R + MA and a swollen portion of MP provides the rma-mp brace (Fig. 16); MA apical branch not clearly discernible from RP branches; AA3 +4 near base arched to meet shortly with $\mathrm{CuP}$; and sinusoid AP3 +4 runs basally close and in parallel to AP1+2 (all apomorphies); 17 - Philopotamidae: Dolophilodes aequalis Knowlton \& Harnson, 1939, fore- and hind wing. MA branch separates apically distinctly from RP branches in both wings; mp-cua brace is short fusion in fore wing but plesiomorphic cross-vein brace in hind-wing (two plesiomorphies).

roptera and basal Mecoptera (Notiothaumidae) (Fig. 10) the two branches separate at the base and run closely and in parallel for most of the wing length. The narrow area between the two con- tains very short, obversely oblique ra-rp cross-veins. In Raphidioptera (Fig. 9) and more derived Mecopterida (Figs 12, 13), veinal branches are few and further apart, but in some Trichop- 


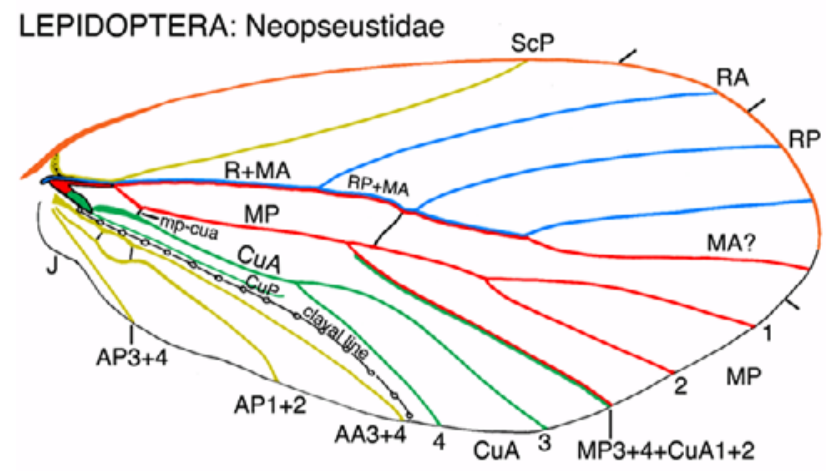

Fig. 18. Lepidoptera, hind wing: Neopseustidae: Neopseustis meyricki Hering, 1925. Features similar to Trichoptera, but fusion of $\mathrm{CuA} 1+2$ with $\mathrm{MP} 3+4$ obscured $(\mathrm{CuA} 1+2$ crossing towards MP3 +4 is almost always reduced); CuP partly reduced (an apomorphy); abutment of $\mathrm{CuP}$ to $\mathrm{AA} 3+4$ near base obscured; AP3+4 straight (all apomorphies). CuP runs anterior to the claval furrow (a plesiomorphy).

tera (Fig. 15) some obversely oblique cross-veins are frequently retained.

34. RP branches: $\mathbf{0}$, variously, dichotomously branched, not long; 1, densely pectinate, parallel and long. In most groups, RP divides dichotomously several times, while branches are neither dense nor parallel. In Megalopera, Neuroptera and basal Mecoptera (Notiothaumidae) (Figs 5-10), RP branches are extra long, densely arranged in a pectinate series, and parallel, dividing into narrow forks. In Raphidioptera, the RP branches become reduced and irregular. In derived Mecoptera with narrow wings and reduced venation, RP branches are fewer and further apart, and the original pectinate arrangement is often obscured.

35. RP1 in Coleoptera: 0, well developed; 1 , reduced to short stump or absent. In non-Coleoptera, RP1 is usually branched; it is absent only rarely when RP or RP1+2 remains unbranched. In Coleoptera and Strepsiptera, RP1 is expressed as a simple, curved vein (Figs 19-22, 28). State 0 occurs in most Staphyliniformia and in scattered members of Scirtoidea, Elateriformia, Bostrichoidea and Cucujiformia. State 1 occurs in Archostemata, Adephaga and Myxophaga.

36. $R$ and MA at wing base: 0 , not fused; 1 , fused. In Orthoneoptera and Pleconeoptera, R and MA are not fused basally. In Blattoneoptera, Hemineoptera and Endoneoptera, MA fuses at base with R and then with RP, and either visibly separates again from RP apically [as in Neuroptera: Dilaridae (Fig. 6), and Raphidioptera (Fig. 9)], or its separation is uncertain because medial and radial branches cannot be distinguished. This important, long fusion occurs only in groups with the partial anojugal lobe.

37. $\mathbf{R}$ and MP at base: 0 , approximate or contiguous; 1, separated by membranous zone. In Blattoneoptera, Hemineoptera, Hymenoptera, Neuropterida and Mecopterida, R and MP are close together at the base. In Coleoptera and Strepsiptera, they are separated by broad membranous zone. In Strepsiptera, $\mathrm{R}$ and MP immediately diverge broadly, while in Coleoptera, they are connected by a strong cross-vein brace rpma-mp called the medial bridge (see Definitions). Orthoneoptera have been coded as inapplicable for this character, since MP is not present at the wing base (only the stem of M).

38. Radial and medial basivenalia: $\mathbf{0}$, articulated or fused; $\mathbf{1}$, separated by membranous zone. In Orthoneoptera, Blattoneop- tera, Hemineoptera, Hymenoptera, Neuropterida and Mecopterida, the radial basivenale (BR) and medial basivenale (BM) are articulated, annexed or fused. In Coleoptera and Strepsiptera (Figs 29-31), they are separated by a membranized, flexible strip, which in Strepsiptera includes part of BR and all of BM. In Coleoptera this is one of the important features in apical folding.

39. "Y"-shaped fusing brace RP + MA + MP: 0, absent; 1 , present. In Orthoneoptera, Blattoneoptera, Hemineoptera, Coleoptera, Strepsiptera, Neuropterida and Mecopterida, there is no "Y" shaped fusion of RP + MA and MP. In Hymenoptera, $\mathrm{RP}+\mathrm{MA}$ and MP approach one another at nearly a right angle and fuse into a long, "Y"-shaped fusing brace (see lines of circles in Figs 3 and 4). This "Y" vein is clearly indicated in some Xyelidae (Pleroneura bruneicornis, Fig. 4), but it becomes more or less membranized in the rest of Hymenoptera (Xyela nearctica, Fig. 3), thus profoundly confusing homologization of hymenopteran wing venation with that in other Endoneoptera.

40. Reinforced radio-medial cross-veins: 0 , absent; 1 , two, usually long; 2, one, usually short. In non-Coleoptera, rp-mp cross-veins, if present, are short and weak. In Archostemata, Adephaga and Myxophaga (Figs 20-22), there are two reinforced cross-vein braces, which are usually relatively long and enclose the oblongum cell. In Polyphaga (Fig. 19), there is only one reinforced brace, which is usually quite short and forms with RP the medial hook.

41. Rpma-mp brace: 0, absent; 1, present. In Orthoneoptera, Blattoneoptera, Hemineoptera and Hymenoptera, a radio-medial cross-vein brace close to the wing base is absent. In Coleoptera, Strepsiptera, Neuropterida and Mecopterida, it is present and variously expressed: as a long, flexible cross-vein brace (medial bridge) connecting RA, RP + MA and MP (in Coleoptera, Fig. 31), a long strip of thickened flexible membrane between RA and RP + MA (in Strepsiptera, Figs 29, 30), a long, sinuous cross-vein brace rpma-mp or ma-mp (in Neuropterida), a strong bar-like rma-mp cross-vein (in Diptera, Fig. 33), a strip of thickened membrane connecting R + MA to MP and sometimes also to $\mathrm{Cu}$ (in Trichoptera), or by MP abutting $\mathrm{R}+\mathrm{MA}$ (in Mecoptera and Lepidoptera).

42. Mp-cua brace (arculus): 0 , absent; 1 , a cross-vein to fusion brace, MP and $\mathrm{CuA}$ distinct; 2, a fusion brace, adjacent portions of MP and CuA membranized. The mp-cua brace, also known as the arculus, is the most frequent and most homoplasious brace in insect wings. It is absent in fossil and extant Orthoneoptera, which have instead a unique fusion of $\mathrm{CuA}$ starting at base, with the entire stem of $\mathrm{M}$ and a large portion of MP (Fig. 2). It is absent in ancestral Pleconeoptera (Liomopterida) and replaced by an analogous brace m-cua in Plecoptera + Embioptera (Kukalová-Peck, 1991, Fig. 6.19A). It is absent in ancestral Blattoneoptera (Carpenter, 1992), in modern Isoptera (Mastotermes), and in the fore wings of modern Blattodea and Mantodea. A groundplan with the brace mp-cua (i.e. present in both wings and shared by all extinct and extant representatives) (state 1) occurs only in Hemineoptera and Endoneoptera (a synapomorphy). In Hemineoptera, Neuropterida and Coleoptera (Figs 23-26) the arculus is usually a short cross-vein, but in the last group it is often obscured by a fold. In Mecopterida it is a very short to moderately long fusion brace, which in some Trichoptera becomes obscured by the medio-cubital flexion line (Figs 15, 16). In Hymenoptera, the fusion is long and the adjacent portions of $\mathrm{CuA}$ and $\mathrm{MP}$ are membranized (only partly membranized in some Xyelidae, Figs 3,4) so that the fusion brace mp-cua becomes part of a "new" vein composed of MP, the brace, and $\mathrm{CuA}$. Strepsiptera, which lack this brace (Fig. 28 ), are coded as state 0 , although it is likely that this represents a secondary loss. 

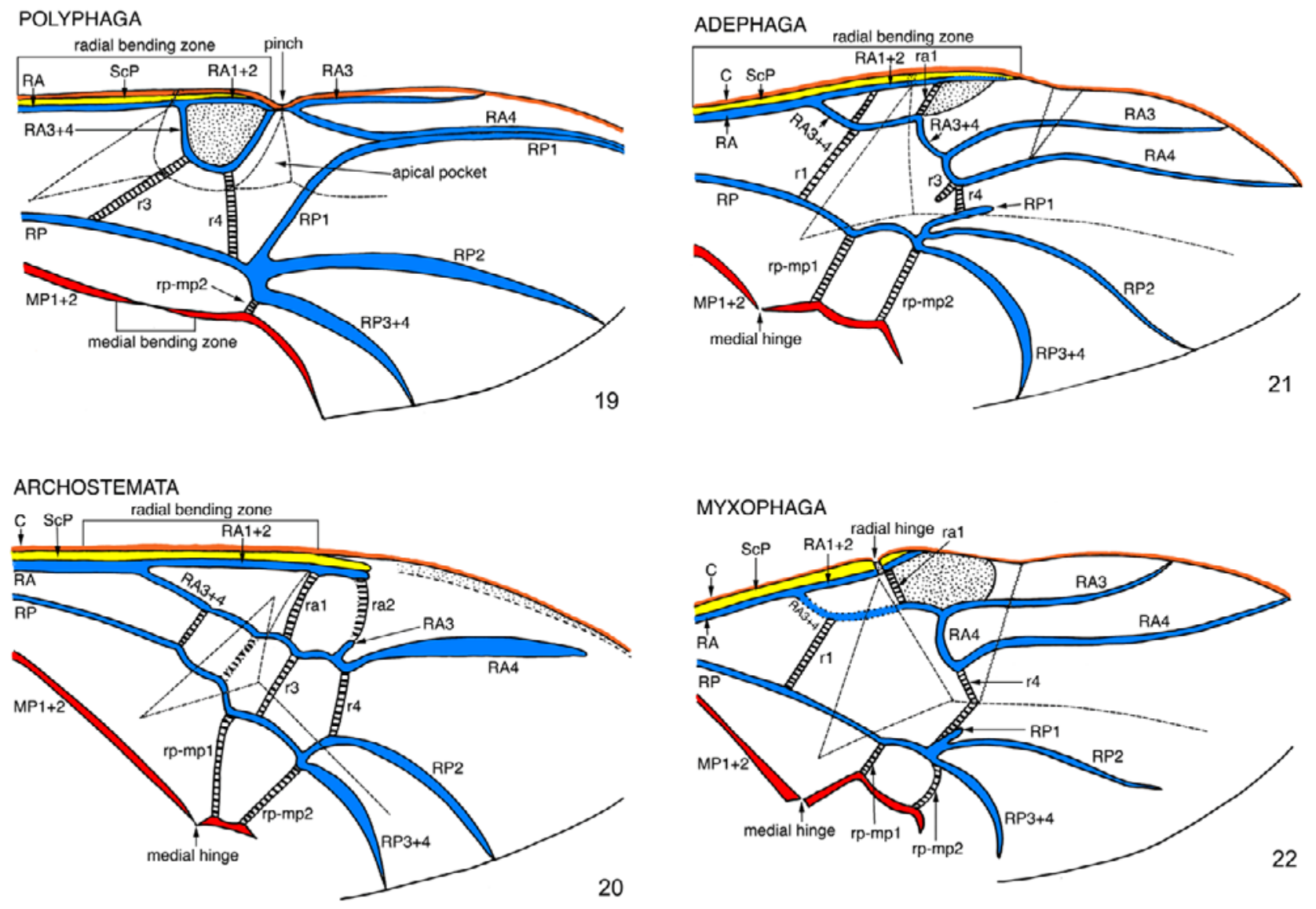

Figs 19-22. Coleoptera hind wing distal part, differences in subordinal groundplans. 19 - Polyphaga; 20 - Archostemata; 21 Adephaga; 22 - Myxophaga. Groundplans include the most plesiomorphic veinal character states found among the taxa listed in Appendix 1, including all enhanced cross-veins (indicated by cross-hatching) involved in the apical folding.

Remarks to Figs 19-26 and 31. Coleoptera hind wing. Venation groundplan: Veinal sectors RA and RP are basally completely separated in primitive (or very shortly annexed in derived) taxa; fusion MP4 + CuA1 near posterior margin short, very basal; anal fold long and active in folding (three plesiomorphies). RP + MA and MP connected near base by medial bridge (a cross-vein brace present also in Neuropterida and Diptera) (a synapomorphy shared with Neuropterida + Mecopterida). MP basally separate and desclerotized; mp-cua brace (arculus) crossed by fold and usually lost; RA-RP widely diverging basally; RP branches arched; MA apically not discernible from RP branches; claval flexion line lost (six synapomorphies shared with Strepsiptera). Numerous autapomorphies connected with the apical wing folding. Articulation groundplan: tegula reduced; humeral plate + subcostal + radial basivenale $+\mathrm{ScA}$ bulge fused into a plate; radial and medial basivenale separated by a membranized zone; $2 \mathrm{Ax}$ body posterior corner narrowed, elongated; 3Ax composed of a plate (goblet-heel + saucer + cubital + anal + jugal arm fused) and a separate $3 \mathrm{Ax}$ fragment bearing muscular insertions; $3 \mathrm{Ax}$ articulated directly with the cubital basivenale (all synapomorphies shared with Strepsiptera). Median plate subdivided, and 2Ax body articulated directly with the median plate (two apomorphies).

43. MP-CuA fusion near posterior wing margin: 0 , absent; 1, present. In non-Endoneoptera, MP and $\mathrm{CuA}$ branches do not fuse together close to the posterior wing margin. The fusion occurs typically in the wings of Endoneoptera (Figs 5-17, 23-26). In the hind wings of most Lepidoptera (Fig. 18) and of all Strepsiptera (Fig. 28), the CuA veinal crossing (a portion of vein between $\mathrm{CuA}$ and MP) is absent, and thus there is usually no indication of a fusion between the two systems. This is considered to be a secondary loss in both groups. In modern Neuropterida, crossing of CuA towards MP cannot be distinguished from regular cross-veins. For purposes of the analysis, Strepsiptera are coded as inapplicable.

44. MP-CuA fusion in Endoneoptera: 0, simple, short, involving MP4 and $\mathrm{CuA} 1 ; \mathbf{1}$, involving MP4 and $\mathrm{CuA} 1+2 ; \mathbf{2}$, involving $\mathrm{MP} 3+4$ and $\mathrm{CuA} 1+2 ; \mathbf{3}$, involving $\mathrm{MP} 3+4, \mathrm{CuA} 1+2$ and a portion of $\mathrm{CuA}$. This fusion between the most proximal branch of MP and the most distal branch of $\mathrm{CuA}$ in the vicinity of the posterior wing margin is present in Neuropterida, Mecopterida, Coleoptera (hind wings only) and Hymenoptera. The individual branches involved may vary within a group or between groups according to size, folds and other factors. The shortest possible fusion between two simple primary branches (state 0 in Endoneoptera) occurs in Neuropterida (Figs 5-9), basal Polyphaga (Fig. 23) and Archostemata (Fig. 24), while in basal Mecopterida (Fig. 10), MP4 fuses with CuA1+2 (state 1). An extensive fusion of $\mathrm{CuA} 1+2$ and $\mathrm{MP} 3+4$ (state 2 ) is a synapomorphy of Myxophaga and Adephaga (Figs 25, 26). The most complex fusion involving MP3+4, a portion of $\mathrm{CuA}$ and $\mathrm{CuA} 1+2$, occurs in Hymenoptera (state 3) (Figs 3, 4). Strepsiptera, which lack the "crossing" of CuA towards MP, is coded as inapplicable, along with basal neopteran taxa. 
POLYPHAGA

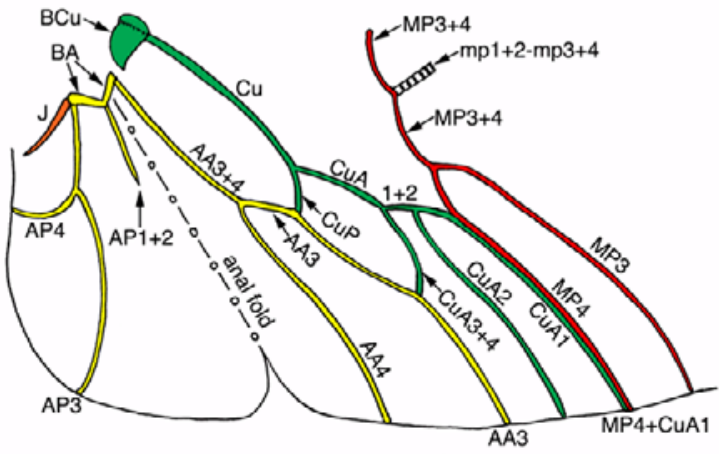

23

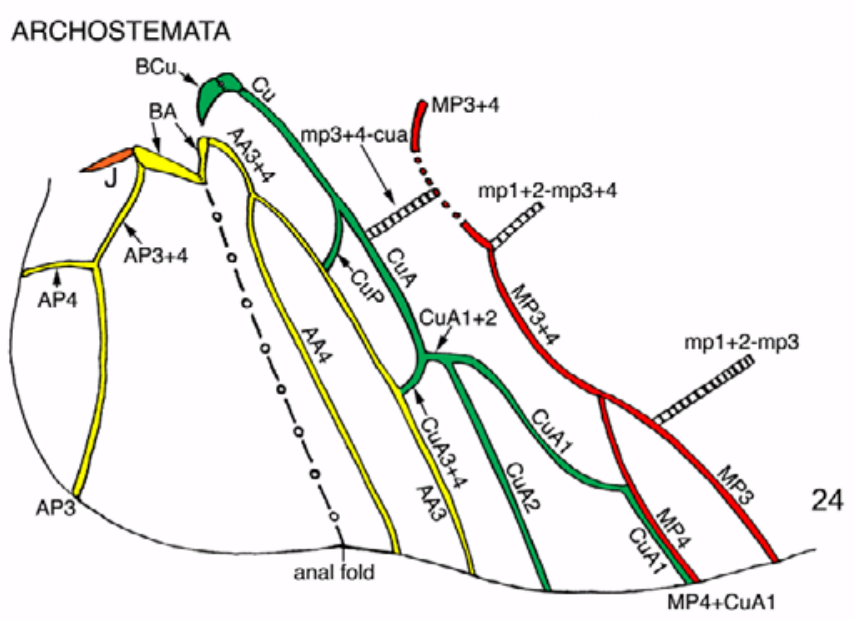

\section{ADEPHAGA}

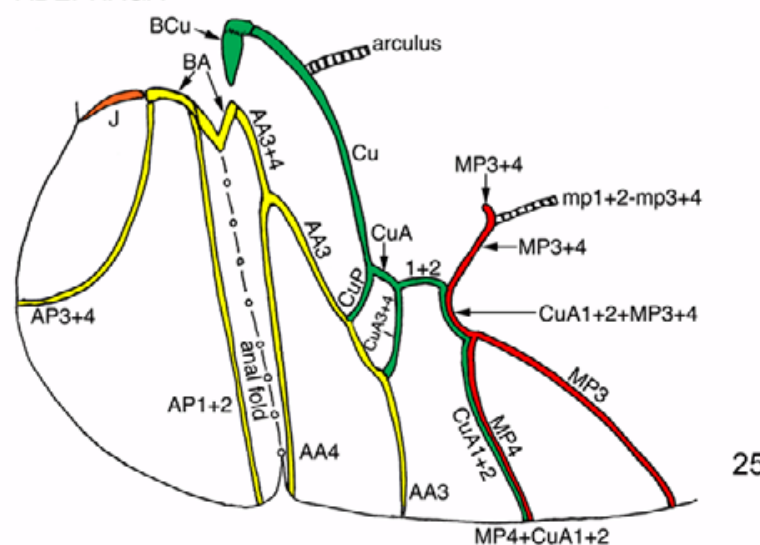

MYXOPHAGA

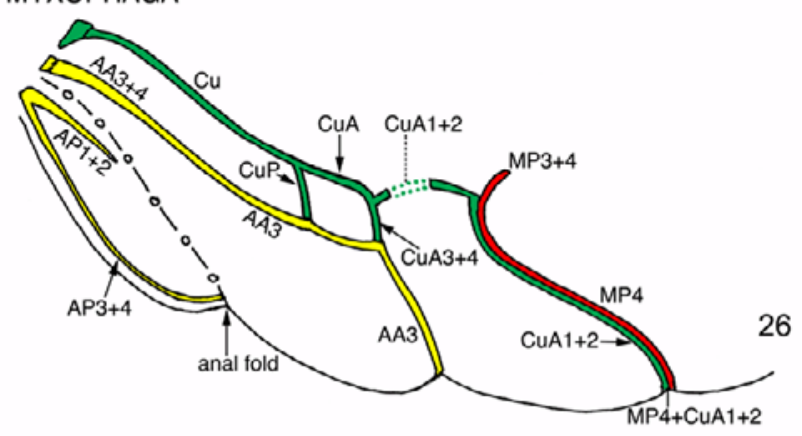

Figs 23-26. Coleoptera hind wing proximal part, differences in subordinal groundplans. 23 - Polyphaga; 24 - Archostemata; 25 Adephaga; 26 - Myxophaga. Groundplans include the most plesiomorphic veinal character states found among the taxa listed in Appendix 1, including all enhanced cross-veins (indicated by cross-hatching) involved in the apical folding. See remarks at Figs $19-22$.

45. CuA2 in Coleoptera: 0, present; 1 , absent. Within the order Coleoptera, $\mathrm{CuA} 2$ is present in Archostemata (Ommatidae, some Cupedidae) and in a number of basal Polyphaga (Fig. 23), but has been lost in Adephaga (Fig. 25) and Myxophaga (Fig. 26). This character has been coded as inapplicable in non-Coleoptera, although it may be present in those taxa with a dichotomously branched $\mathrm{CuA}$.

46. Connection between cubital and anal systems: 0 , absent; $1, \mathrm{CuA} 3+4$ ending on $\mathrm{AA} 1+2 ; \mathbf{2}, \mathrm{CuP}$ and $\mathrm{CuA} 3+4$ both curved, ending on AA3; 3, AA $3+4$ connected with or shortly annexed to $\mathrm{CuP} ; 4$, AA3+4 fused basally with $\mathrm{Cu}$. In nonEndoneoptera, these systems are not connected (state 0 ). In Hymenoptera (Figs 3, 4), they are connected by the distal portion of $\mathrm{CuA} 3+4$, curving and ending on $\mathrm{AA} 1+2$ (state 1 ). In Coleoptera (Figs 23-26), $\mathrm{CuP}$ and also $\mathrm{CuA} 3+4$ both curve and end on AA3 (state 2). In Neuropterida and Mecopterida (Figs 5-14), AA3+4 is connected with CuP by cross-vein braces, or annexed to it for a short distance (with a claval flexion line running between the veins) in some Raphidioptera, Mecoptera and Trichoptera (state 3) (Figs 9-17); the veins are close but independent in Lepidoptera (Fig. 18). In Strepsiptera (Figs 28-30), $\mathrm{AA} 3+4$ fuses basally with undivided cubitus $\mathrm{Cu}$ (state 4 ).

47. AA1+2: 0, branched; 1, simple, long, strong; 2, simple, short, weak; 3, absent. In Orthoneoptera (Fig. 2), AA1+2 divides into branches, as do all other anal veins. It is simple, weakened and gradually lost in Blattoneoptera (present only in basal Blattodea) (Fig. 2) and present as a long, simple, strong vein in Hymenoptera (Figs 3, 4). In Hemineoptera, Coleoptera, Strepsiptera, Neuropterida and Mecopterida, AA1+2 is absent.

48. AA3+4: 0, branched or simple, straight; 1, simple, curved concavely (towards AP) or undulate; 2 , simple, arched to become annexed to $\mathrm{CuP}$. In Orthoneoptera, Blattoneoptera, Hymenoptera, Coleoptera, Strepsiptera and Neuropterida, AA $3+4$ is branched or simple and almost straight. In Hemineoptera (Fig. 2), AA3+4 is simple and curved concavely towards AP or undulate (state 1). In Mecopterida (Mecoptera, Trichoptera) (Figs 10-17), AA3+4 is simple and distinctly arched proximally to become annexed for a short distance to the claval line with $\mathrm{CuP}$ running along its bottom (state 2). In Lepidoptera (Fig. 18), this arch is lost.

49. AA4 in Coleoptera: 0, present; 1 , absent. AA4 is a primary branch present in the full anojugal lobe of Orthoneoptera. In groups with a partial anojugal lobe, $\mathrm{AA} 3+4$ is undivided and AA4 absent in Blattoneoptera, Hemineoptera and Strepsiptera. Among the Endoneoptera, AA3+4 is divided and AA4 present in Neuropterida (Megaloptera and Raphidioptera) (Figs 7-9), and in Coleoptera (Figs 23-25), it is present in all suborders except Myxophaga.

50. AP3+4: 0, dichotomously branched or forked; 1, simple. In non-Coleoptera, AP3+4 is branched or forked in basal members of all groups, except Strepsiptera, where it is always undivided. Within Coleoptera, AP3 +4 forms a broad fork (state 0 ) in Polyphaga (Fig. 23) and Archostemata (Fig. 24), but is simple (state 1) in Adephaga and Myxophaga (Figs 25, 26). 


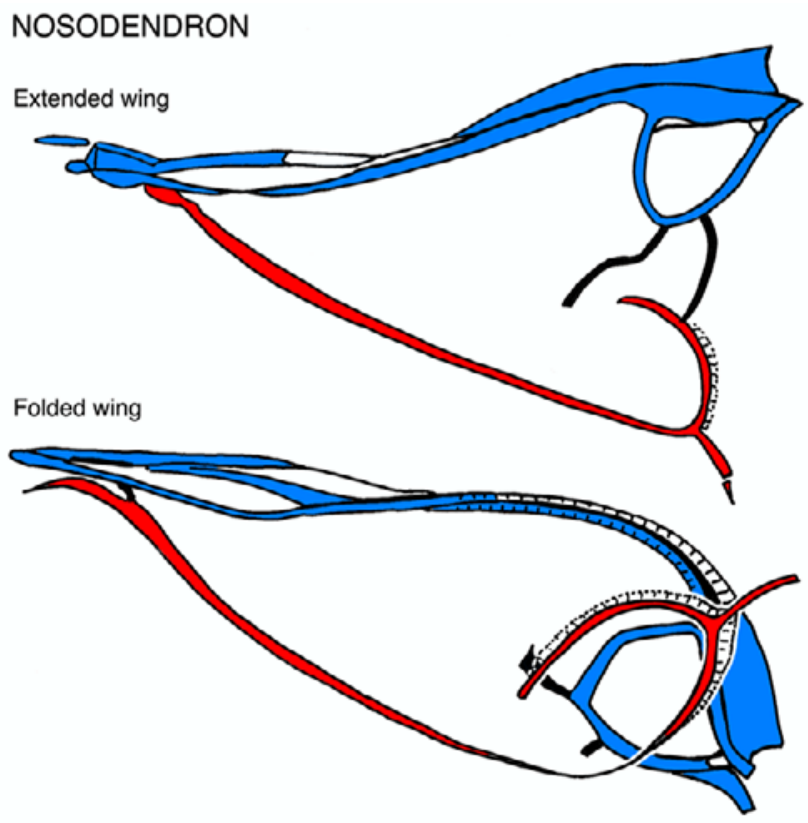

Fig. 27. Polyphaga hind wing, folding diagram. Bostrichoidea: Nosodendridae: Nosodendron unicolor Say, hind wing extended and folded; radial bar and loop (blue), medial bar and loop (red), cross-veins (black). In a folded wing, both bars develop mildly curved bending zones; radial zone shows crimping, medial zone changes position from horizontal to perpendicular. Drawings of folded and unfolded wings were based on different specimens.

51. Radial bending zone: 0 , absent; 1 , present. In basal Neoptera, Hymenoptera, Neuropterida and Mecopterida, as well as Strepsiptera, the radial bending zone is absent. In Coleoptera, the anterior wing strut or radial bar in basal members of Polyphaga, Archostemata, Adephaga and Myxophaga contains a longer or shorter region of flexibility, which is capable of bending in one plane only and is prevented from bending by a rotation of the wing strut. Beutel \& Haas (2000) considered this region to be present in Adephaga, Myxophaga and staphyliniform Polyphaga only; however, well developed bending zones occur in various other basal polyphagans (Scirtoidea and Bostrichoidea, Fig. 27), as well as Ommatidae in the Archostemata. In derived Archostemata (Cupedidae and Micromalthidae) and in some Myxophaga, this bending zone may be transformed into a more abrupt hinge.

52. Medial bending zone: 0 , absent; 1 , present; 2 , replaced by a hinge. In basal Neoptera, Hymenoptera, Neuropterida and Mecopterida, as well as Strepsiptera, the medial bending zone is also absent. In basal Polyphaga (Fig. 19), such as Scirtoidea and Bostrichoidea, the posterior wing strut or medial bar may also have a bending zone, which may have crimping in larger forms but usually consists of a flattened or weakened area; this zone has been lost in Staphyliniformia, as well as in more derived groups such as Elateriformia and Cucujiformia. In Archostemata, Adephaga and Myxophaga (Figs 20-22) the bending zone has been transformed into an abrupt medial hinge.

53. Medial loop: 0 , absent; 1 , formed by oblongum cell; 2 , formed by medial hook. In all groups except Coleoptera, the area between RP + MA and MP is relatively narrow, often with several equally short, weak cross-veins. In the Coleopteran suborders Archostemata, Myxophaga and Adephaga (Figs 20-22),

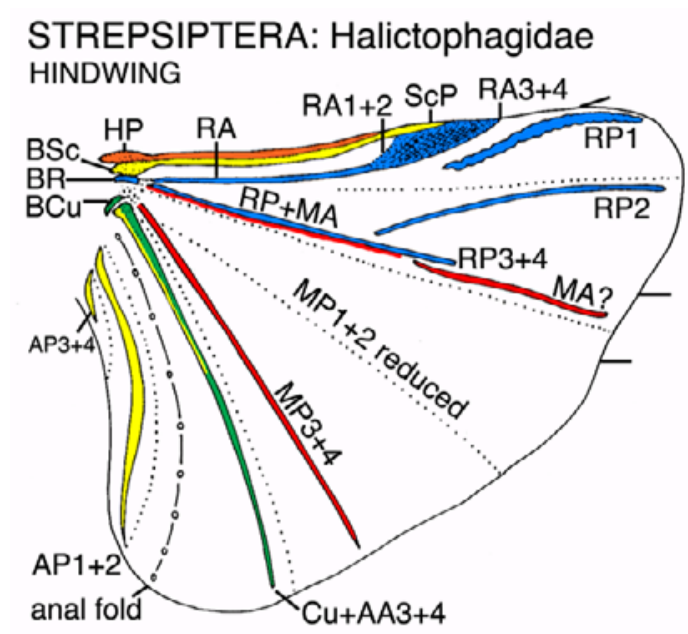

Fig. 28. Strepsiptera, hind wing. Halictophagidae: Coriophagus rieki Kinzelbach, 1971. Veinal features as in Coleoptera including: radial bar indicated but incomplete; the stem of $\mathrm{R}$ absent; RA-RP sectors basally very broadly diverging; medial bridge replaced by a strip of stiffened membrane; RP branches curved; claval flexion line lost; and MP basally separate from RP + MA, membranized. Autapomorphies: Uniquely shaped broadly triangular hind wings; veins highly reduced, not reaching posterior wing margin; most braces and all cross-veins lost. Note that the radial arrangement of veins and veinal areas identifies strepsipteran metathoracic wings safely as hind wings, same as in the other Neoptera. Neopteran fore wings bear strongly diminished jugal area, sunken and folded under a much larger anal area, anal bar is present, and $\mathrm{AA} 3+4+\mathrm{AP}$ are fused basally into a short stem. Some strepsipteran fore wings show remnants of anal bar and a small, sunken jugal area (personal observation by JKP).

this area is broad, with two enhanced cross-veins, a longer rp-mp1 and a shorter rp-mp2, enclosing a large, rigid cell called the oblongum. MP1+2 is strengthened to form a posterior wing strut called the medial bar by Kukalová-Peck \& Lawrence (1993), which forms the posterior end of this cell. In Polyphaga (Fig. 19), cross-vein rp-mp1 is suppressed and rp-mp2 shortened to join the end of RP with the bases of RP1, RP2, RP3 and the medial bar, thus forming a narrow, pliable, coiled section (Fig. 27) called the medial hook by Kukalová-Peck \& Lawrence (1993) and the m-cu loop by Crowson (1955 and elsewhere).

54. Triangular folding area: 0 , absent; 1, present, not bisecting RA3+4; 2, present, bisecting RA3+4. In all nonColeoptera, the triangular folding area is absent. In Polyphaga (Fig. 19), it is present but usually small, not bisecting RA3+4, and it is variously suppressed in derived taxa. In Archostemata, Myxophaga and Adephaga (Figs 20-22), it is large with one angle extending anteriorly so that RA $3+4$ is bisected in two places. This is not as obvious in Micromalthus and Lepicerus where the basal portion of RA3+4 has been obliterated. In all Archostemata, most Myxophaga and some Adephaga, the basoposterior angle of this folding area also bisects RP in two places.

55. Triangular folding area (and r1): 0 , not close to crossvein $\mathrm{r} 1 ; \mathbf{1}$, very close to $\mathrm{r} 1$ which supports its proximal side. In Polyphaga, Archostemata and Myxophaga (Figs 19, 20, 22), the triangular folding area is either far distad from $\mathrm{r} 1$, or $\mathrm{r} 1$ is reduced (in Polyphaga). In Adephaga (Fig. 21), the proximal 


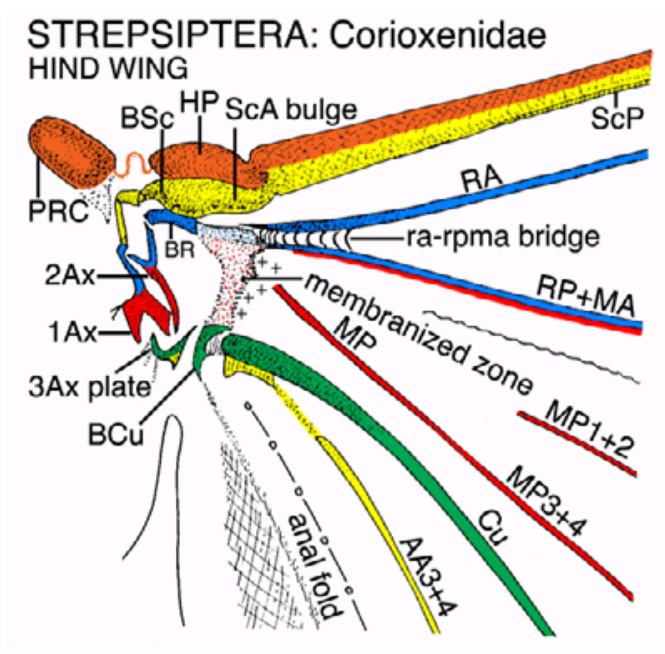

Fig. 29. Strepsiptera, hind wing base: Corioxenidae: Triozocera mexicana Pierce, 1909. Features of pteralia shared solely with Coleoptera include: tegula lost; basivenalia BR + BSc + ScA bulge + humeral plate fused into a large sclerotized plate; similar shape of $2 \mathrm{Ax}$ body and arm; membranized zone separating radius from media; $3 \mathrm{Ax}$ forms a plate; $3 \mathrm{Ax}$ plate includes cubital arm; $3 \mathrm{Ax}$ articulated directly with $\mathrm{BCu} ; 3 \mathrm{Ax}$ fragment inserted with wing flexor muscle sometimes present. Apomorphies: trianglular wing, unique veinal reductions, median plate and PWP lost. Note: ScP hidden in a groove under the anterior margin and $\mathrm{AA} 3+4$ is fused with $\mathrm{Cu}$ only basally.

side of the triangle runs close to and is reinforced by the long, enhanced $\mathrm{r} 1$.

56. Body of $2 A x: 0$, large, forming strongly sclerotized triangle; $\mathbf{1}$, diminished, with proximal rim thickened and curved, distally desclerotized. In Orthoneoptera and Pleconeoptera, the $2 \mathrm{Ax}$ body resembles a large, strongly sclerotized triangle, (state 0) (Fig. 34A-E). In Blattoneoptera, Hemineoptera and Endoneoptera, the $2 \mathrm{Ax}$ body becomes smaller, with the proximal rim curved and often thickened, and the distal part more or less desclerotized (state 1) (Fig. 34F-R).

57. Body of $2 \mathrm{Ax}$ (form): $\mathbf{0}$, forming large, sclerotized triangle; 1, with proximal rim curved and thickened, distal portion concentrically creased; $\mathbf{2}$, with proximal rim curved, very thick, distal portion bulging; $\mathbf{3}$, forming short, broad, twisted triangle; 4, forming long, slender, posteriorly tapering triangle. In Orthoneoptera and Pleconeoptera, the $2 \mathrm{Ax}$ body is large, robust and triangular (state 0) (Fig. 34A-E). In Blattoneoptera, the 2Ax body resembles a human ear with the proximal rim curved and thickened, and the distal portion concentrically creased (state 1) (Fig. 34F-I), with Dermaptera (Fig. 34 F) representing the most divergent type. In Hemineoptera, the curved proximal rim is very thick and the bulging distal portion is connected with the medial plate (FM) (state 2) (Fig. 34J). In Hymenoptera, Neuropterida and Mecopterida, the $2 \mathrm{Ax}$ body is triangular, short, broad and twisted (state 3) (Fig. 34K, N-R). In Coleoptera and Strepsiptera, the $2 \mathrm{Ax}$ triangle is longer, slender and tapering posteriorly, while the thickened rim is absent (state 4) (Fig. 34L-M).

58. 2 Ax proximo-ventral projection: 0 , absent; 1 , present. In most Coleoptera, the proximal edge of $2 \mathrm{Ax}$ bears a broad, flat ventral projection, which extends proximad under the body of $1 \mathrm{Ax}$ and strongly restricts the motion of $2 \mathrm{Ax}$ against $1 \mathrm{Ax}$, so that the two sclerites function as a single plate (Hörnschemeyer, 1998). This feature is absent in all other orders and also in most

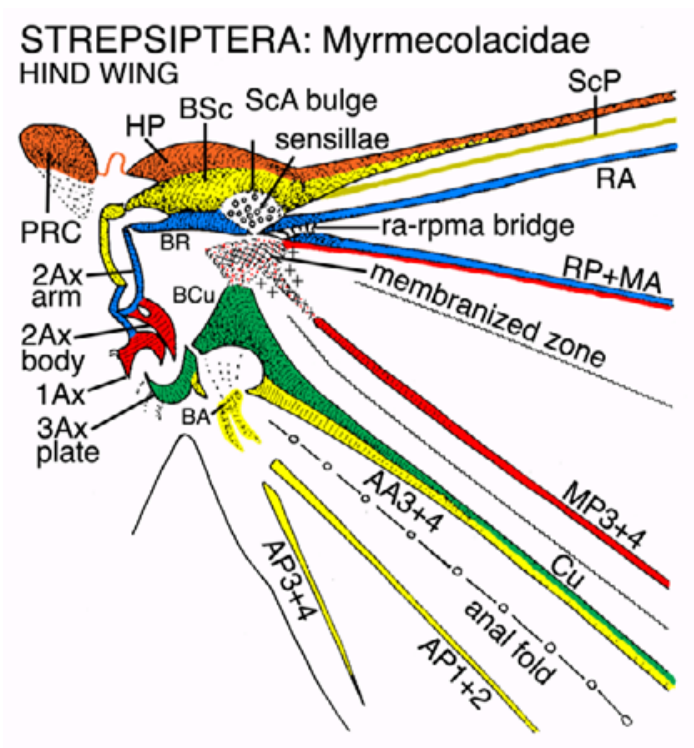

Fig. 30. Strepsiptera, hind wing base: Myrmecolacidae: Caenocholax fenyesi Pierce, 1909. Similar as in Corioxenidae, but $\mathrm{ScP}$ not hidden under the anterior margin and $\mathrm{AA} 3+4$ is fused to $\mathrm{Cu}$.

Archostemata; however its presence in Ommatidae suggests that it is a basal condition for Coleoptera.

59. 2Ax arm: 0, relatively short, without anterior lobe; 1, enlarged, broadly fused to BSc, 2, with anterior lobe projected towards BSc; $\mathbf{3}$, long and thin, without anterior lobe. The second axillary sclerite bears an arm, which may be simple or have an anterior lobe extending towards the subcostal basivenale BSc. In Plecoptera, Orthoneoptera, most Blattoneoptera, and in Hymenoptera, the arm is short (compared with the body), and has no lobe (state 0) (Fig. 34B-E, G-I, K), but in Orthoneoptera, it is always fused with 2Ax body and in Dermaptera (Fig. $34 \mathrm{~F}$ ) it has become elongated. In Hemineoptera, it is enlarged, irregularly shaped and fused with the subcostal basivenale (state 1) (Fig. 34J). In Neuropterida and Mecopterida, the arm is relatively massive and bears an anterior lobe, which may articulate with the base of BSc (state 2) (Fig. 34N-R). In Strepsiptera and Coleoptera, the arm is very long and thin and lacks an anterior lobe (state 3) (Fig. 34L-M).

60. 3Ax (composition): 0, composed of five articulated sclerites; $\mathbf{1}$, composed of three articulated sclerites; $\mathbf{2}$, consisting of single plate. In the homologized all-pterygote system of nomenclature (Kukalová-Peck, 1983, 1997; Haas \& Kukalová-Peck, 2001, Table 6), 3Ax is an axillary cluster composed of a goblet and heel (cubital axalare $\mathrm{AXCu}$ subdivided by a fold), saucer (anojugal axalare AXAJ), and three arms: cubital, anal and jugal (cubital fulcalare $\mathrm{FCu}$, anal fulcalare $\mathrm{FA}$, and jugal fulcalare FJ). As $3 \mathrm{Ax}$ rotates, the anal arm, which is articulated to the largest basivenale BAA $3+4$, folds the anojugal fan into resting position. The cubital proxalare $(\mathrm{PRCu})$, located proximad of $3 \mathrm{Ax}$ (in the "window" into which 3Ax collapses), is expressed as a desclerotized strip connected to the $3 \mathrm{Ax}$ goblet (Fig. 35F, $\mathrm{H}, \mathrm{J}, \mathrm{K}$ ) or as a weakly sclerotized sclerite (Fig. $35 \mathrm{~N}, \mathrm{O}$ ). The $3 \mathrm{Ax}$ fragment, present in Coleoptera and in some Strepsiptera (Fig. 35L-N), is located more posteriorly than PRCu and usually has two muscles attached to it in beetles and one only in Strepsiptera. This suggests that it may have become detached from the $3 \mathrm{Ax}$ goblet. The cubital arm of $3 \mathrm{Ax}(\mathrm{FCu})$ is independent in Neoptera but it often becomes adjacent to the median 


\section{COLEOPTERA: ARCHOSTEMATA: Ommatidae}

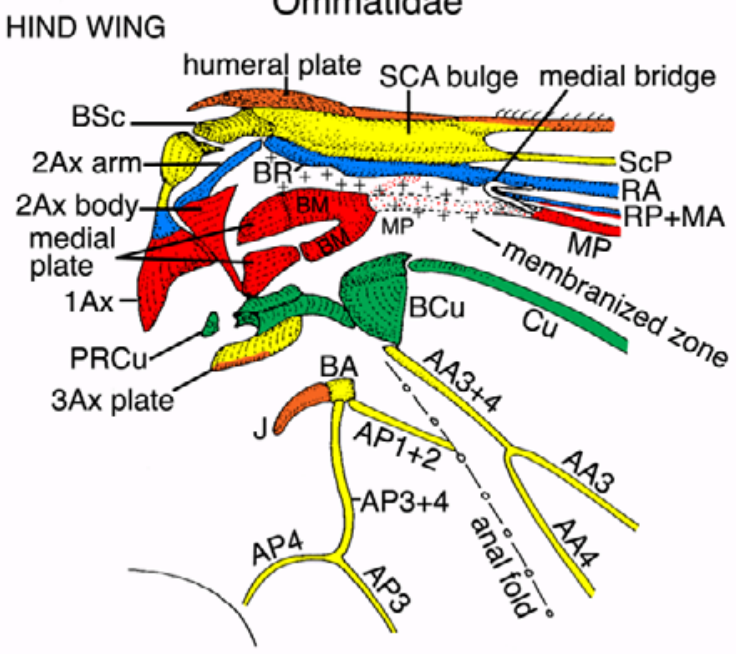

Fig. 31. Coleoptera, Archostemata, hind wing base. Ommatidae: Omma stanleyi Newman, 1839. Most features as in Strepsiptera (Fig. 29), but PRC smaller, median plate FM is uniquely subdivided, PWP/4Ax present, 3Ax fragment always present, BM less membranized, medial bridge is a cross-vein brace, venation is much less reduced and it contains many attributes used in apical folding. See remarks at Figs 19-22.

plate (FM) in a derived state. The anal arm is articulated with basivenale BAA $3+4$ directly or, after its fusion with $3 \mathrm{Ax}$ saucer in Endoneoptera, by strips of stiffened membrane. The jugal arm is often reduced (Fig. 35, marked as FJ; AXJ marked by orange line). In basal Plecoptera, Orthoneoptera (Fig. 35A-E) and Blattoneoptera (Fig. $35 \mathrm{~F}-\mathrm{H}$ ), 3Ax contains up to five movably articulated sclerites (state 0). In Blattoneoptera, the anal arm is adapted to various enlargements of the partial anojugal lobe: it is fused with a secondarily elongated jugal arm in Dermaptera (Fig. 35F), broadly fused with the jugal arm in Mantodea (Fig. 35G), robust and protruding distally in basal Blattodea (Fig. $35 \mathrm{H}$ ), and robust and very broad in Isoptera (Fig. 35I). In Hemineoptera, Hymenoptera, Neuropterida and Mecopterida, 3Ax is composed of three articulated sclerites: the goblet $\&$ heel, the saucer with the anal and jugal arms fused to it, and the (articulated) cubital arm (state 1) (Fig. 35J, K, N-R). In Coleoptera and Strepsiptera, the goblet and heel, saucer and three arms become fused into a single plate (state 2) (Fig. 35L-M). Hörnschemeyer (1998, Fig. 4) suggested that 3Ax started as a single plate and fragmented subsequently, but this hypothesis is not supported by all-pterygote evidence, on which the groundplan must be built.

61. Basalare, projection and knob: 0 , absent; 1, small and inconspicuous; 2, large and protruding. In non-Endoneoptera, the basalare does not bear a dorsal projection with a knob at its apex. In Endoneoptera, this projection is present, fits under the subcostal bulge and serves to lock the wings at rest in a flexed position. In Hymenoptera, the basalare and knob are small; consequently the subcostal bulge fits over the dorsal half of basalare in the fore wings, and over the entire basalare in the hind wings (Hörnschemeyer, 2002, p. 25). In Coleoptera, Neuropterida and Mecopterida, the basalar projection is elongated and the knob is very large so that the subcostal bulge fits only over the greatly enlarged knob. Note that Hörnschemeyer considered this latter

\section{MECOPTERA: Panorpidae HIND WING}

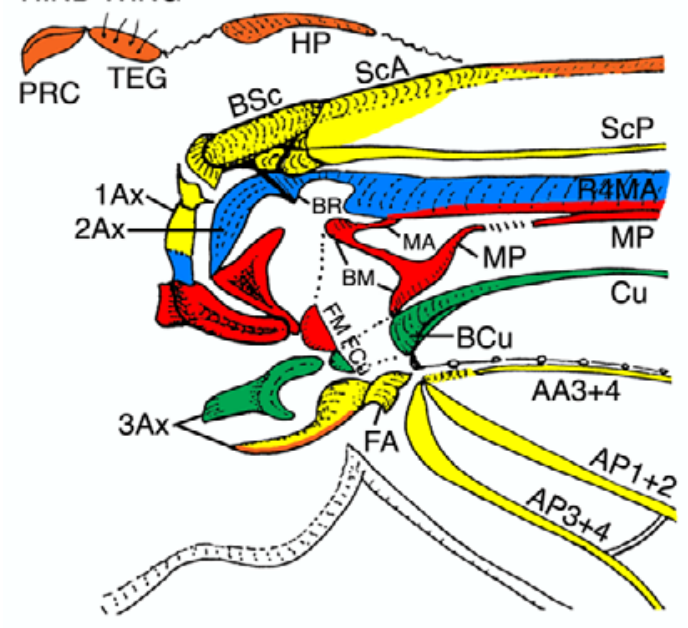

Fig. 32. Mecoptera, hind wing base. Panorpidae: Panorpa maculosa Hagen, 1861. Features similar as in Diptera (Fig. 33) but different from Strepsiptera (Figs 28-30) include: PRC small (large in Strepsiptera), tegula present (lost in Strepsiptera); BSc and BR articulated (not fused as in Strepsiptera); basivenalia $\mathrm{BR}$ and $\mathrm{BM}$ articulated (not separated by a mebranous zone as in Strepsiptera); fusion BSc $+\mathrm{BR}+\mathrm{ScA}$ bulge $+\mathrm{HP}$ absent (present in Strepsiptera); 2Ax body short, twisted triangle (long, pointed in Strepsiptera); 2Ax arm broad, lobed towards BSc (thin in Strepsiptera); FM and $\mathrm{FCu}$ abut, form median plate ( $\mathrm{FCu}$ fused with $3 \mathrm{Ax}$ in Strepsiptera); $3 \mathrm{Ax}$ goblet and saucer are loosely articulated (not fused into a $3 \mathrm{Ax}$ plate as in Strepsiptera); and $3 \mathrm{Ax}$ does not articulate with cubital basivenale $\mathrm{BCu}$ (as it does in Strepsiptera). There are no features besides those in the endoneopteran groundplan shared with Strepsiptera.

\section{DIPTERA: Tabanidae FORE WING}

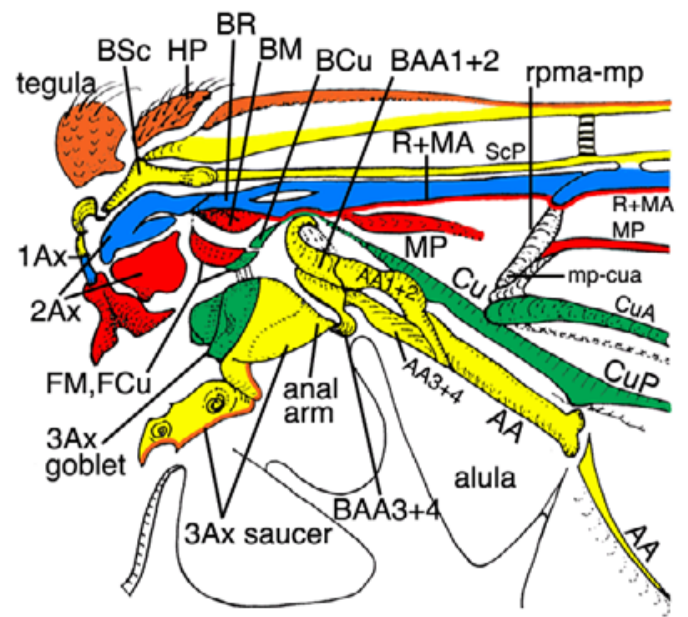

Fig. 33. Diptera, fore wing base. Tabanidae: Tabanus atratus Fairchild, 1960. Pteralia in fore wings are similar to those in Mecoptera hind wings (Fig. 32), but 3 Ax goblet and saucer are compacted; and saucer is very long with an added hinge (two apomorphies). There are no features shared with Strepsiptera hind wing. 
PLECOPTERA ORTHOPTERA

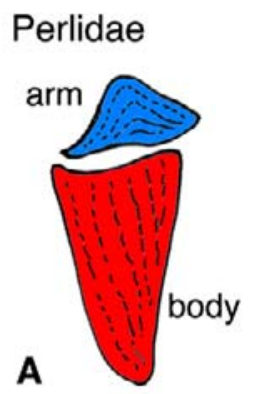

DERMAPTERA

$\mathbf{F}$

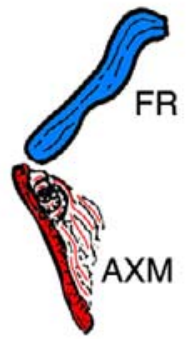

Tettigoniidae

B

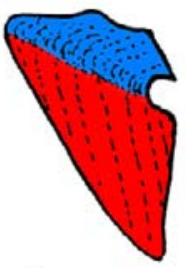

Gryllacrididae

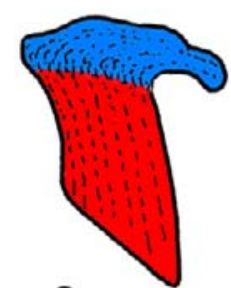

C
PHASMATODEA

Acrididae

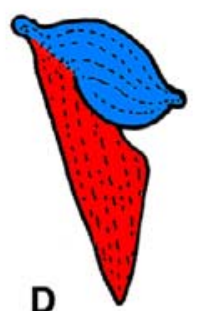

Panoplidae

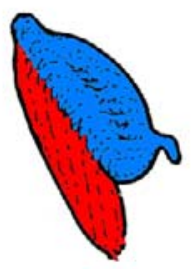

E

MANTODEA BLATTODEA ISOPTERA

\section{FULGOROMORPHA HYMENOPTERA}

\section{COLEOPTERA}

\section{STREPSIPTERA}
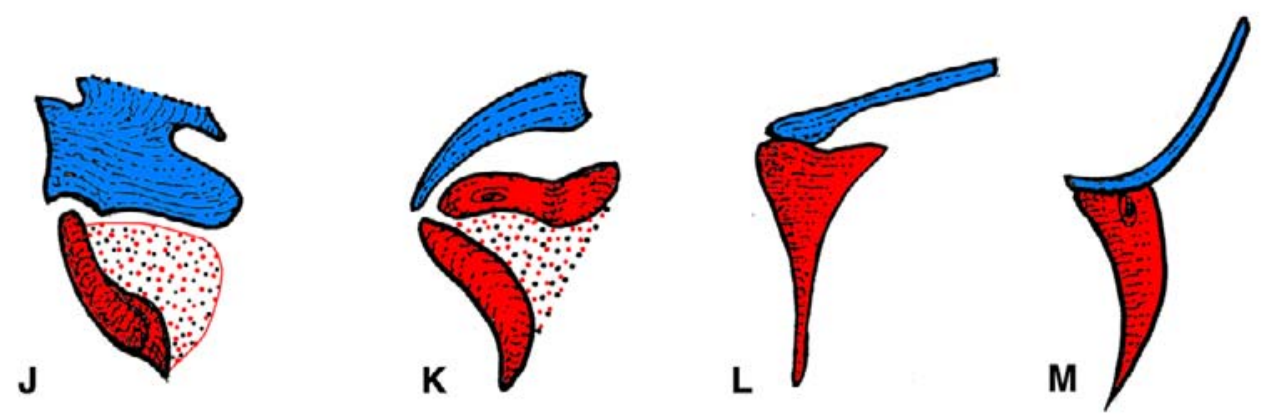

NEUROPTERA

TRICHOPTERA MECOPTERA

DIPTERA
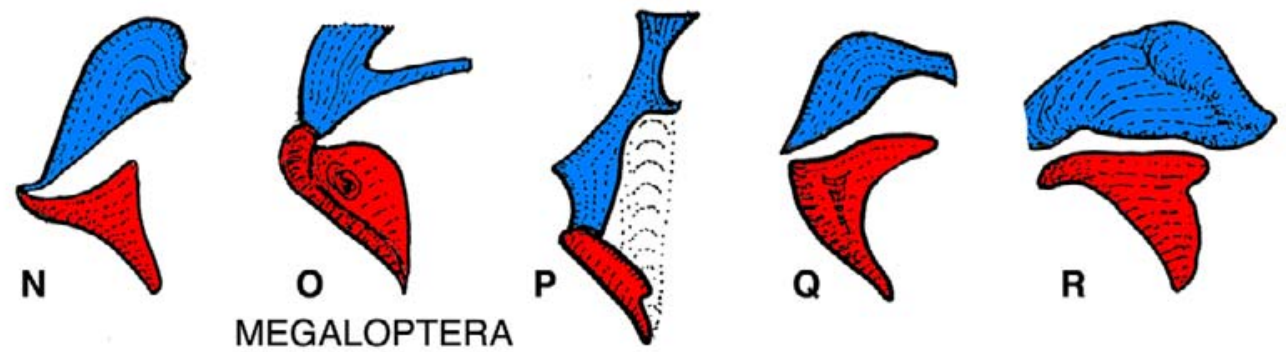

Fig. 34. Neopteran 2Ax. A-E - Plecoptera, Orthoptera: 2Ax body triangular, large, robust; $2 \mathrm{Ax}$ arm robust but short; $2 \mathrm{Ax}$ arm free in basal Plecoptera (fused with body in derived taxa), arm always fused with body in Orthoptera; F-I - Blattoneoptera: $2 A x$ body curved, distally membranized; arm slim and short, long in Dermaptera; F - Diplatyidae, G - Mantidae, H - Blaberidae, I Mastotermitidae; J - Hemineoptera: Fulgoromorpha: Copidocephalidae: 2Ax highly autapomorphic, body with thick, curved proximal rim (thinner, enlarged distal portion may extend towards median plate); 2Ax arm large, irregularly shaped, anteriorly fused with BSc; K - Hymenoptera: Xyelidae: 2Ax body short, twisted triangle with a membranized centro-distal portion; 2Ax arm robust, short, anterior lobe absent; L - Coleoptera: Ommatidae and M - Strepsiptera, Myrmecolacidae: 2Ax body a slim, posteriorly narrowing triangle; 2Ax arm slim, very long. N-R - Neuropterida, Mecopterida: 2Ax body small twisted triangle; 2Ax arm broad, longer than body width, with a robust anterior lobe extended towards BSc (often articulated with BSc); N - Rapismatidae, $\mathrm{O}$ - Corydalidae, $\mathrm{P}$ Limnephilidae, Q - Panorpidae, R - Tipulidae. Scars on 2Ax body sometimes mark the contact with the ventral wing process VWP. 

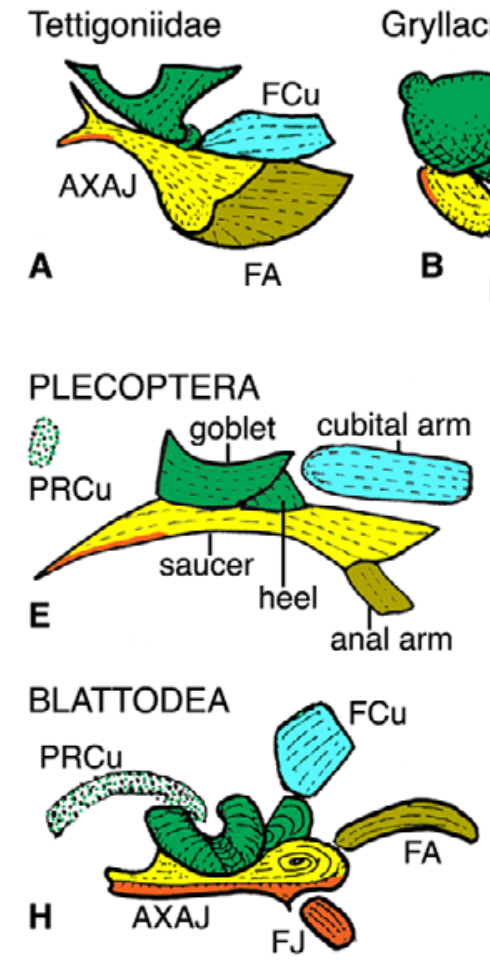
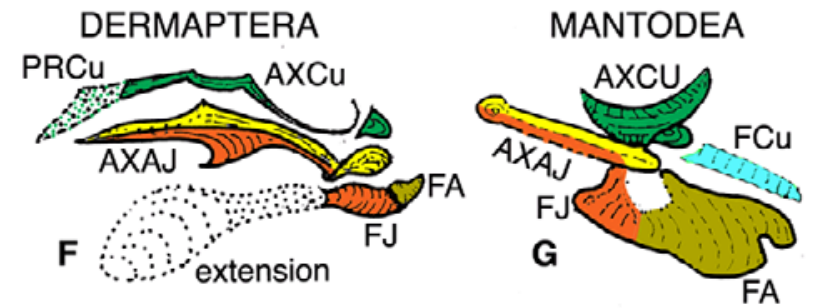

ISOPTERA
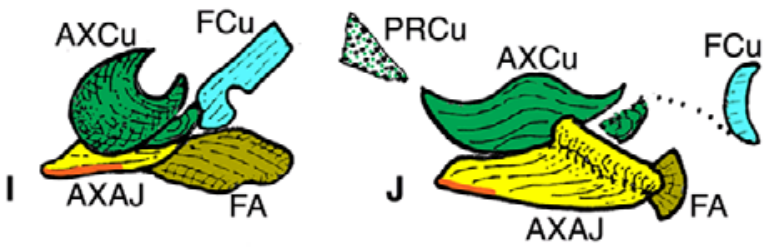

Paloplidae

FULGOROMORPHA
HYMENOPTERA

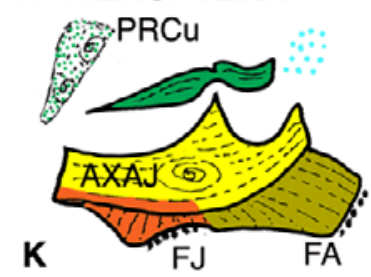

COLEOPTERA

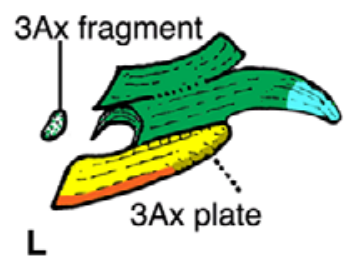

STREPSIPTERA MEGALOPTERA

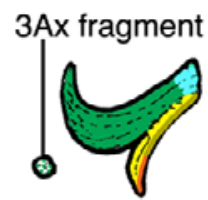

M

$\Leftrightarrow P R C u$

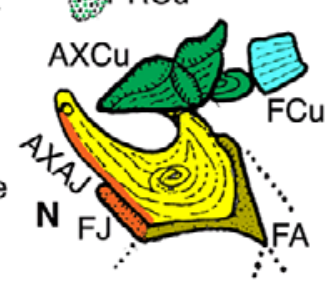

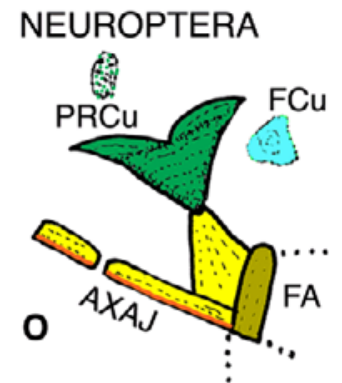

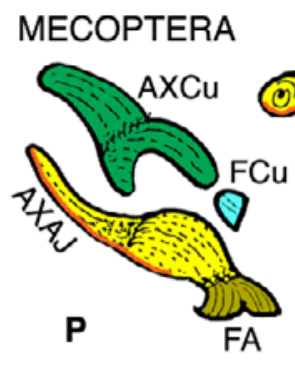

DIPTERA

TRICHOPTERA
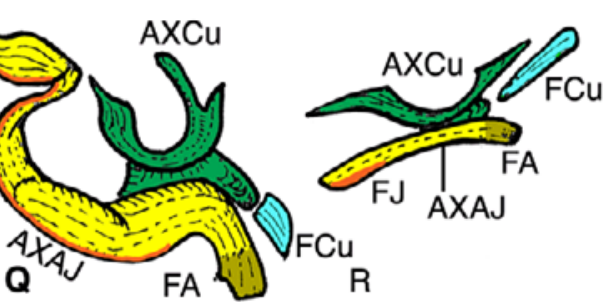

Fig. 35. Neopteran 3Ax. A-E - Orthoptera and Plecoptera: 3Ax jugal arm reduced (jugal system of the full anojugal lobe is articulated directly with the tergum or PWP, by varied secondary jugal struts: JKP, unpublished observation); E - Perlidae. F-I - Blattoneoptera: AXJ part of 3Ax saucer and jugal arm mostly well developed; goblet robust; F - Diplatyidae: goblet-heel and saucer separated; G - Mantidae: FA + FJ fused, large; H - Blaberidae: FA robust and very long; I - Mastotermitidae: goblet strongly protruding, FA robust, FJ reduced. J - Fulgoromorpha: Copidocephalidae: FA fused with 3Ax saucer jutting distally, FJ reduced. K-R Endoneoptera: 3Ax goblet develops two almost symmetrical "wings"; FA and FJ fused with 3Ax saucer; anal area is connected with 3 Ax by stiffened membrane (strips marked by dotted lines in Coleoptera, Neuropterida). K - Hymenoptera: Xyelidae: FA and FJ relatively very large, plesiomorphic, fused to saucer with suture. L-M - Coleoptera: Ommatidae and Strepsiptera: Myrmecolacidae: $3 \mathrm{Ax}$ changes into a plate including all three arms; $3 \mathrm{Ax}$ fragment with flexor muscle insertions separates from 3Ax (in Coleoptera and some Strepsiptera). N-O - Neuropterida: Megaloptera: Corydalidae and Neuroptera: Rapismatidae: saucer forms a narrow "neck" articulated with goblet; FA and FJ form narrow rim on saucer. P-R - Mecopterida: P - Mecoptera: Panorpidae: goblet and saucer disconnected; FA fused at the distal end of saucer; Q - Diptera: Tipulidae: goblet broadly articulated with saucer; saucer very long, with folds; FA narrow, terminally placed; R - Trichoptera: Limnephilidae: 3Ax goblet and saucer slim; FA narrow, terminally placed. 3Ax saucer may bear distally a concentric scar, but flexor muscles are inserted more proximally (or into 3Ax fragment). Green, yellow and orange in separate or fused axalaria indicates their alignment with cubital, anal and jugal vein, respectively. Green dots mark retention of weakened cubital proxalare PRCu in basal taxa. Light blue denotes cubital arm (cubital fulcalare FCu) as present, light blue dots, as lost (in Hymenoptera). 


\begin{tabular}{|c|c|c|c|c|c|c|c|c|c|c|c|}
\hline CHARACTER & ORTH & BLAT & HEMI & HYME & $\mathrm{MECO}$ & NEUR & STRE & POLY & $\mathrm{ARCH}$ & MYXO & ADEP \\
\hline 0. Flight mechanism & 0 & 0 & 1 & 1 & 1 & 1 & 2 & 2 & 2 & 2 & 2 \\
\hline 1. Hind wing apex & 0 & 0 & 0 & 1 & 0 & 0 & 2 & 0 & 0 & 0 & 0 \\
\hline 2. Wing flight units & 0 & 1 & 1 & 1 & 1 & 1 & 1 & 1 & 1 & 1 & 1 \\
\hline 3. Anojugal lobe (veinal support) & 0 & 1 & 1 & 1 & 1 & 1 & 1 & 1 & 1 & 1 & 1 \\
\hline 4. Partial anojugal lobe (intercalaries) & & 1 & 0 & 0 & 0 & 0 & 0 & 0 & 0 & 0 & 0 \\
\hline 5. Anojugal lobe (extent) & 0 & 1 & 2 & 3 & 4 & 4 & 4 & 4 & 4 & 4 & 4 \\
\hline 6. Anojugal lobe (posterior limits) & 0 & 0 & 0 & 0 & 1 & 1 & 1 & 1 & 1 & 1 & 1 \\
\hline 7. Anojugal lobe in hind wing & 0 & 0 & 0 & 0 & 1 & 1 & 0 & 0 & 0 & 0 & 0 \\
\hline 8. Claval flexion line (expression) & 0 & 1 & 1 & 1 & 1 & 1 & 1 & 1 & 1 & 1 & 1 \\
\hline 9. Claval flexion line (Endoneoptera) & & & & 0 & 1 & 1 & 2 & 2 & 2 & 2 & 2 \\
\hline 10. Anal fold (expression) & 0 & 1 & 1 & 1 & 2 & 2 & 1 & 1 & 1 & 1 & 1 \\
\hline 11. Anal fold (crossing AP1+2) & 0 & 0 & 1 & 0 & 0 & 0 & 0 & 0 & 0 & 0 & 0 \\
\hline 12. Tegula & 0 & 0 & 0 & 0 & 0 & 0 & 1 & 1 & 1 & 1 & 1 \\
\hline 13. Anterior margin near wing base & 0 & 0 & 0 & 0 & 0 & 0 & 1 & 1 & 1 & 1 & 1 \\
\hline 14. Anterior margin near wing apex & 0 & 0 & 0 & 0 & 0 & 1 & 0 & 0 & 0 & 0 & 0 \\
\hline 15. ScA & 0 & 1 & 2 & 3 & 3 & 3 & 3 & 3 & 3 & 3 & 3 \\
\hline 16. ScA bulge (broad, convex) & & & & 0 & 1 & 1 & 1 & 1 & 1 & 1 & 1 \\
\hline 17. ScA bulge (oblique, groove) & & & 0 & 1 & 0 & 0 & 0 & 0 & 0 & 0 & 0 \\
\hline 18. ScP (ending) & 0 & 0 & 0 & 0 & 0 & 0 & 1 & 1 & 1 & 1 & 1 \\
\hline 19. BSc, BR \& base of ScA & 0 & 0 & 0 & 0 & 0 & 0 & 1 & 1 & 1 & 1 & 1 \\
\hline 20. Pterostigma (cross-veins) & 0 & 0 & 0 & 0 & 0 & 0 & 0 & 0 & 1 & 1 & 1 \\
\hline 21. Radial cross-veins & 0 & 0 & 0 & 0 & 0 & 0 & 2 & 1 & 1 & 1 & 1 \\
\hline 22. Cross-vein r1 in Coleoptera & & & & & & & & 1 & 0 & 0 & 0 \\
\hline 23 Cross-vein r3 in Coleoptera & & & & & & & & 0 & 0 & 1 & 1 \\
\hline 24. Radial cell & 0 & 0 & 0 & 0 & 0 & 0 & 0 & 1 & 0 & 0 & 0 \\
\hline 25. RA forking & 0 & 0 & 0 & 0 & 0 & 0 & 1 & 1 & 1 & 1 & 1 \\
\hline 26. RA3+4 in Coleoptera & & & & & & & & 0 & 0 & 1 & 0 \\
\hline 27. RA3-RA4 fork in Coleoptera & & & & & & & & 0 & 1 & 1 & 1 \\
\hline 28. RA3+4 \& RA3 in Coleoptera & & & & & & & & 0 & 0 & 0 & 1 \\
\hline 29. RA3 & 0 & 0 & 0 & 0 & 0 & 0 & 0 & 1 & 2 & 1 & 1 \\
\hline 30. RA4 (fusion with RP1) & 0 & 0 & 0 & 0 & 0 & 0 & 0 & 2 & 1 & 1 & 1 \\
\hline 31. RA3 and RA4 (divergence) & 0 & 0 & 0 & 0 & 0 & 0 & 0 & 0 & 1 & 1 & 1 \\
\hline 32. RP branches & 0 & 0 & 0 & 1 & 0 & 0 & 2 & 2 & 2 & 2 & 2 \\
\hline 33. RA and RP (close, parallel) & 0 & 0 & 0 & 0 & 1 & 1 & 0 & 0 & 0 & 0 & 0 \\
\hline 34. RP branches (pectinate) & 0 & 0 & 0 & 0 & 1 & 1 & 0 & 0 & 0 & 0 & 0 \\
\hline 35. RP1 in Coleoptera & & & & & & & & 0 & 1 & 1 & 1 \\
\hline 36. R \& MA at wing base & 0 & 1 & 1 & 1 & 1 & 1 & 1 & 1 & 1 & 1 & 1 \\
\hline 37. R \& MP at base & & 0 & 0 & 0 & 0 & 0 & 1 & 1 & 1 & 1 & 1 \\
\hline 38. R \& M basivenalia & 0 & 0 & 0 & 0 & 0 & 0 & 1 & 1 & 1 & 1 & 1 \\
\hline 39. "Y"-shaped RP + MA + MP & 0 & 0 & 0 & 1 & 0 & 0 & 0 & 0 & 0 & 0 & 0 \\
\hline 40. Reinforced r-m cross-veins & 0 & 0 & 0 & 0 & 0 & 0 & 0 & 2 & 1 & 1 & 1 \\
\hline 41. Rpma-mp brace & 0 & 0 & 0 & 0 & 1 & 1 & 1 & 1 & 1 & 1 & 1 \\
\hline 42. Mp-cua brace (arculus) & 0 & 0 & 1 & 2 & 1 & 1 & 0 & 1 & 1 & 1 & 1 \\
\hline 43. MP-CuA fusion near margin & 0 & 0 & 0 & 1 & 1 & 1 & & 1 & 1 & 1 & 1 \\
\hline 44. MP-CuA fusion (Endoneoptera) & & & & 3 & 1 & 0 & & 0 & 0 & 2 & 2 \\
\hline 45. $\mathrm{CuA} 2$ in Coleoptera & & & & & & & & 0 & 0 & 1 & 1 \\
\hline 46. Anal-cubital connection & 0 & 0 & 0 & 1 & 3 & 3 & 4 & 2 & 2 & 2 & 2 \\
\hline 47. AA $1+2$ & 0 & 2 & 3 & 1 & 3 & 3 & 3 & 3 & 3 & 3 & 3 \\
\hline 48. $\mathrm{AA} 3+4$ & 0 & 0 & 1 & 0 & 2 & 0 & 0 & 0 & 0 & 0 & 0 \\
\hline 49. AA4 in Coleoptera & & & & & & & & 0 & 0 & 1 & 0 \\
\hline 50. $\mathrm{AP} 3+4$ & 0 & 0 & 0 & 0 & 0 & 0 & 1 & 0 & 0 & 1 & 1 \\
\hline 51. Radial bending zone & 0 & 0 & 0 & 0 & 0 & 0 & 0 & 1 & 1 & 1 & 1 \\
\hline 52. Medial bending zone & 0 & 0 & 0 & 0 & 0 & 0 & 0 & 1 & 2 & 2 & 2 \\
\hline 53. Medial loop & 0 & 0 & 0 & 0 & 0 & 0 & 0 & 2 & 1 & 1 & 1 \\
\hline 54. Triangular folding area & 0 & 0 & 0 & 0 & 0 & 0 & 0 & 1 & 2 & 2 & 2 \\
\hline 55. Triangular area (and r1) & & & & & & & & 0 & 0 & 0 & 1 \\
\hline 56. Body of $2 \mathrm{Ax}$ & 0 & 1 & 1 & 1 & 1 & 1 & 1 & 1 & 1 & 1 & 1 \\
\hline 57. Body of $2 \mathrm{Ax}$ (form) & 0 & 1 & 2 & 3 & 3 & 3 & 4 & 4 & 4 & 4 & 4 \\
\hline 58. 2 Ax proximo-ventral projection & 0 & 0 & 0 & 0 & 0 & 0 & 0 & 1 & 1 & 1 & 1 \\
\hline 59. $2 \mathrm{Ax}$ arm \& anterior lobe & 0 & 0 & 1 & 0 & 2 & 2 & 3 & 3 & 3 & 3 & 3 \\
\hline 60. 3Ax (composition) & 0 & 0 & 1 & 1 & 1 & 1 & 2 & 2 & 2 & 2 & 2 \\
\hline 61. Basalare projection \& knob & 0 & 0 & 0 & 1 & 2 & 2 & 2 & 2 & 2 & 2 & 2 \\
\hline 62. Posterior wing process & 0 & 0 & 0 & 0 & 0 & 0 & 0 & 0 & 1 & 0 & 1 \\
\hline
\end{tabular}




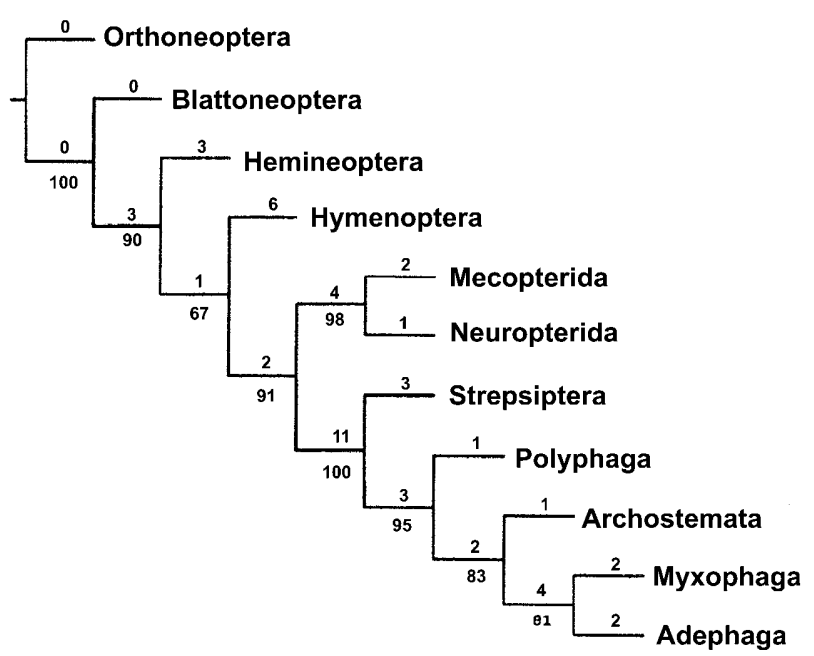

Fig. 36. Phylogenetic relationships: the single most parsimonious tree based on 63 hind wing characters (tree length $=99$, $\mathrm{CI} \& \mathrm{RI}=0.96$ ). Branch lengths are above the lines and Bootstrap values below them.

state as plesiomorphic and its much smaller version in Hymenoptera as an autapomorphy (a reduction). It is quite possible that the basalare in ancestral Endoneoptera was larger than in modern Hymenoptera, nevertheless the condition appears to be quite significantly closer to the groundplan level than a unique, greatly enlarged BA knob at the end of a long projection.

62. Posterior wing process (PWP): 0, short and at least proximally broad; $\mathbf{1}$, long, narrow and distally expanded. The posterior wing process is homologous with $4 \mathrm{Ax}$, which like all other articular sclerites, is part of the wing structure and is originally articulated with the tergum (Stenzhorn, 1974; KukalováPeck, 1983). Although 4Ax is present as a separate sclerite in basal members of some orders, it is very often secondarily fused with the tergum and transformed into the posterior wing process. In most Neoptera, 4Ax/PWP is short to moderately long, and broad at least at "base" (proximal edge, which may or may not be fused with tergum); this is also the case in the coleopteran suborders Myxophaga and Polyphaga. In Archostemata and Adephaga (Figs 75 and 78 in Kukalová-Peck \& Lawrence, 1993), however, PWP is long, narrow and expanded at the apex. We interpret this as a synapomorphy and the condition in Myxophaga as an autapomorphic reduction. Hörnschemeyer (1998, 2002) considered the condition in Archostemata and Adephaga to be plesiomorphic, and the short PWP in Myxophaga and Polyphaga to be a synapomorphy.

\section{Cladistic analysis}

A cladistic analysis of the above data set (Table 1) was carried out using Winclada 0.9.9 (Nixon, 1999) with Nona 2.0 (Goloboff 1999). All characters were considered to be unordered. A single tree was produced (Fig. 36) with a length of 99 , CI and RI of 0.96. Bootstrap values were calculated using Winclada with Nona. The low Bootstrap value for the Archostemata + Myxophaga + Adephaga node may reflect the fact that most of the important hind wing synapomorphies supporting coleopteran suborders are involved in or reflect changes in the mechanism of transverse wing folding; as a result these are coded as unknown or inapplicable for all noncoleopteran taxa.

\section{Nodal support}

Suppport for the various nodes is given below $(\mathbf{U}=$ unambiguous changes only; A \& $\mathbf{D}=$ additional changes using accelerated or delayed transformation, respectively).

Adephaga (U2): 28,1 - RA3+4 and RA3 forming right angle; 55,1 - triangular folding area very close to $\mathrm{r} 1$. (D1): 62,1 - posterior wing process long, narrow and distally expanded (also in Archostemata).

Myxophaga (U2): 26,1 - RA3+4 membranized basally; 49,1 - AA4 absent. (A1): 62,0 - posterior wing process short and at least proximally broad (reversal)

Myxophaga + Adephaga (U4): 23,1 - reinforced cross-vein $\mathrm{r} 3$ highly reduced or absent; 44,2 - MP-CuA fusion involving MP3+4 \& CuA1; 45,1 - CuA2 absent; $\mathbf{5 0 , 1}$ - AP3+4 simple (also in Strepsiptera).

Archostemata (U1): 29,2 - RA3 reduced to short stump. (D1): 62,1 - posterior wing process long, narrow and distally expanded (also in Adephaga).

Archostemata + Myxophaga + Adephaga (U2): 20,1 - pterostigma supported by reinforced cross-veins; 31,1 RA3 and RA4 forming right angle. (A5): 27,1 RA3-RA4 fork far from anterior margin; 35,1 - RP reduced to short stump; 52,2 - medial bending zone replaced by hinge; 54,2 - triangular folding area bisecting RA3+4; 62,1 - posterior wing process long, narrow and distally expanded. (D7): 27,1 - RA3-RA4 fork far from anterior margin; 30,1 - RA4 long, parallel to anterior margin, independent; 35,1 - RP reduced to short stump; 40,1 - reinforced radio-medial cross-veins two, usually long; 52,2 - medial bending zone replaced by hinge; 53,1 - medial loop formed by oblongum cell; 54,2 - triangular folding area bisecting RA3+4.

Polyphaga (U1): 24,1 - radial cell present. (A4): 22,1 - reinforced cross-vein r1 absent; 30,2 - RA2 long, parallel to anterior margin and fused at length with RP1; 40,2 - reinforced medial cross-veins one, usually short; 53,2 medial loop formed by medial hook. (D6): 22,1 - reinforced cross-vein $\mathrm{r} 1$ absent; 30,2 - RA2 long, parallel to anterior margin and fused at length with RP1; 40,2 - reinforced medial cross-veins one, usually short; 52,1 medial bending zone present; 53,2 - medial loop formed by medial hook; 54,1 - triangular folding area very close to $\mathrm{r} 1$ which supports its proximal side.

Coleoptera (U3): 29,1 - RA3 long, parallel to anterior margin; 51,1 - radial bending zone present; 58,1 - 2Ax proximo-ventral projection present. (A5): 30,1 - RA4 long, parallel to anterior margin; 40,1 - reinforced radiomedial cross-veins two, usually long; 52,1 - medial bending zone present; 53,1 - medial loop formed by oblongum cell; 54,1 - triangular folding area present, not bisecting RA3+4. (D2): 21,1 - radial cross-veins forming braces r1, r2, r3, r4; 46,2 - CP and $\mathrm{CuA} 3+4$ both curved, ending on AA3.

Strepsiptera (U3): 1,2 - hind wing apex well in front of anterior edge of wing base; 42,0 - MP-CuA brace absent (reversal); 50,1 - AP3+4 simple (also in Myxophaga + Adephaga). (A2): 21,2 - radial cross-veins 
absent; 46,4 - AA3+4 fused basally with Cu. (D2): 21,2 radial cross-veins absent; 46,4 - AA3+4 fused basally with $\mathrm{Cu}$.

Strepsiptera + Coleoptera (Coleopterida) (U11): 0,2 - fore wings shortened, hind wings flying pair; 12,1 tegula absent; 13,1 - anterior wing margin near base with desclerotized area; 18,1 - ScP ending beyond RA fork and overlapping RA1+2; 19,1 - BSc, BR and base of ScA fused to form plate; $\mathbf{2 5 , 1}-\mathrm{RA}$ forking close to mid wing; 32,2 - RP branches close basally, radiating apically; 37,1 - RP and MP at base separated by membranous zone; 38,1 - radial and medial basivenalia separated by membranous zone; $\mathbf{5 7 , 4}$ - body of $2 \mathrm{Ax}$ forming long, slender, posteriorly tapering triangle; $\mathbf{6 0 , 2}-3 \mathrm{Ax}$ consisting of single plate. (A3): 9,2 - claval flexion line absent; 21,1 radial cross-veins absent; 59,3 - 2Ax arm long and thin, without anterior lobe. (D2): 9,2 - claval flexion line absent; 59,3 - 2Ax arm long and thin, without anterior lobe.

Mecopterida (U2): 44,1 - MP-CuA fusion involving MP4 and $\mathrm{CuA} 1+2 ; \mathbf{4 8 , 2}-\mathrm{AA} 3+4$ simple, arched to become annexed to $\mathrm{CuP}$.

Neuropterida (U1): 14,1 - anterior margin near wing apex with declerotized zone.

Mecopterida + Neuropterida (U4): 7,1 - anojugal lobe in hind wing smaller than in fore wing; 10,2 - anal fold shortened or absent; 33,1 - RA and RP running close and parallel for long distance; 34,1 - RP branches densely pectinate, long. (A1): 46,3 - AA3+4 connected with or shortly annexed to CuP. (D3): 9,1 - claval flexion line shallow and not curved; 46,3 - AA3+4 connected with or shortly annexed to $\mathrm{CuP} ; \mathbf{5 9 , 2}-2 \mathrm{Ax}$ arm with anterior lobe articulated to BSc.

Mecopterida + Neuropterida + Coleopterida (U2): 6,1 - anojugal lobe aligned with or narrow than remigium; 41,1 - Rpma-mp brace present. (A6): 5,4 anojugal lobe much narrower than remigium and ending before basal third; 9,1 - claval flexion line shallow and not curved; 16,1 - ScA bulge broad, convex and heavily sclerotized; 46,2 - $\mathrm{CuP}$ and $\mathrm{CuA} 3+4$ both curved, ending on AA3; 59,2 - 2Ax arm with anteriorlobe arrticulated to BSc; 61,2 - basalare projection and knob large and protruding. (D3): 5,4 - anojugal lobe much narrower than remigium and ending before basal third; 16,1 - ScA bulge broad, convex and heavily sclerotized; 61,2 - basalare projection and knob large and protruding.

Hymenoptera (U6): 1,1 - hind wing apex at same level as anterior edge of wing; 17,1 - ScA bulge distally delimited by deep fold; 32,1 - RP branches reduced to single branch; 39,1 - Y-shaped brace RP + MA + MP present; 42,2 - Mp-cua brace a fusion brace, adjacent portions of MP and $\mathrm{CuA}$ membranized; 47,1 - AA1+2 simple, long, strong. (A1): 44,3 - MP-CuA fusion involving $\mathrm{MP} 3+4, \mathrm{CuA} 1+2$ and a portion of $\mathrm{CuA}$. (D4): 5,3 - anojugal lobe much narrower than remigium and ending between basal third and midwing; 44,3 - MP-CuA fusion involving $\mathrm{MP} 3+4, \mathrm{CuA} 1+2$ and a portion of $\mathrm{CuA}$; 46,1 $-\mathrm{CuA} 3+4$ ending on $\mathrm{AA} 1+2 ; \mathbf{6 1 , 1}$ - basalare projection and knob small and inconspicuous.
Endoneoptera (U1): 43,1 - MP-CuA fusion near posterior margin present. (A5): 5,3 - anojugal lobe much narrower than remigium and ending between basal third and midwing; 15,3 - ScA forming a bulge; 46,1 $\mathrm{CuA} 3+4$ ending on $\mathrm{AA} 1+2 ; \mathbf{5 7 , 3}$ - body of $2 \mathrm{Ax}$ forming short, broad, twisted triangle; 61,1 - basalare projection and knob small and inconspicuous. (D2): 15,3 - ScA forming a bulge; 57,3 - body of 2Ax forming short, broad, twisted triangle.

Hemineoptera (U3): 11,1 - anal fold crossing AP1+2; 48,1 - AA3+4 simple, curved concavely or undulate; 59,1 - 2Ax arm enlarged and broadly fused to BSc. (D3): 5,2 - anojugal lobe much narrower than remigium and ending at about mid wing; 15,2 - ScA reduced; 57,2 - body of $2 \mathrm{Ax}$ with proximal rim curved, very thick, distal portion bulging.

Hemineoptera + Endoneoptera (U3): 0,1 - flight mechanism with fore wings dominant; 42,1 - Mp-cua brace a cross-vein to fusion brace, MP and $\mathrm{CuA}$ distinct; $\mathbf{6 0 , 1}-3 \mathrm{Ax}$ composed of three articulated sclerites. (A4): 5,2 - anojugal lobe much narrower than remigium and ending at about midwing; 15,2: ScA reduced; 47,3 $\mathrm{AA} 1+2$ absent; $\mathbf{5 7 , 2}$ - body of $2 \mathrm{Ax}$ with proximal rim curved, very thick, distal portion bulging. (D1): 47,3 AA $1+2$ absent;

Blattoneoptera: (A1): 4,1 - partial anojugal lobe with pectinate intercalary branches. (D5): 4,1 - partial anojugal lobe with pectinate intercalary branches; 5,1 - anojugal lobe slightly narrower than remigium and ending at or slightly beyond mid wing; 15,1 - ScA forming short, blunt ridge; 47,2 - AA1+2 simple, short, weak; 57,1 body of $2 \mathrm{Ax}$ with proximal rim thickened and curved, distally desclerotized.

Blattoneoptera + Hemineoptera + Endoneoptera: (A10): 2,1 - wing flight units separated by anal fold; 3,1 - anojugal lobe partial, excluding AA; 5,1 - anojugal lobe slightly narrower than remigium and ending at or slightly beyond mid wing; 8,1 - claval flexion line incomplete or absent; 10,1 - anal fold highly convex, long and distinct; 15,1 - ScA forming short, blunt ridge; 36,1 $-\mathrm{R}$ and MA at wing base fused; 47,2 - AA1+2 simple, short, weak; 56,1 - body of $2 \mathrm{Ax}$ diminished with proximal rim thickened and curved, distally desclerotized; $\mathbf{5 7 , 1}$ - body of $2 \mathrm{Ax}$ with proximal rim curved and thickened, distal portion concentrically creased. (D6): 2,1 wing flight units separated by anal fold; 3,1 - anojugal lobe partial, excluding AA; 8,1 - claval flexion line incomplete or absent; 10,1 - anal fold highly convex, long and distinct; 36,1 - R and MA at wing base fused; $\mathbf{5 6 , 1}$ - body of $2 \mathrm{Ax}$ diminished with proximal rim thickened and curved, distally desclerotized.

\section{Results and implications}

This phylogeny differs from what appears to be the commonly accepted one at both superordinal and subordinal levels. In discussions on the relationships among endoneopteran sections (Kristensen, 1975, 1991, 1999) and in ordinal cladograms produced by Wheeler et al. (2001) and Beutel \& Gorb (2001), Hymenoptera and 
Mecopterida are always sister groups, as are Coleoptera and Neuropterida. Our wing characters support the following monophyletic groups: Neuropterida + Mecopterida, and Coleopterida + Neuropterida + Mecopterida.

Our data provide further support for a section Coleopterida including Strepsiptera, the clade Strepsiptera + Coleoptera being supported by 11 unambiguous changes. Yet in the ordinal cladograms produced by Whiting (1998), Whiting \& Wheeler (1994), Whiting et al. (1997) and Wheeler et al. (2001), Strepsiptera and Diptera are always sister groups within the superorder Antliophora of the section Mecopterida. Beutel \& Gorb (2001) also provided support for a monophyletic group consisting of Coleoptera and Strepsiptera, and Kristensen (1999) considered "the question of strepsipteran affinities to remain unanswered".

Within the order Coleoptera, our wing data support the monophyletic groups Archostemata + Myxophaga + Adephaga and Myxophaga + Adephaga, as opposed to Adephaga + Myxophaga + Polyphaga and Myxophaga + Polyphaga proposed by Beutel \& Haas (2000) and Hörnschemeyer (1998, 2002).

\section{DISCUSSION AND CONCLUSION}

\section{Endoneopteran lineages}

Morphological characters used to support phylogenetic hypotheses at the ordinal and supraordinal levels in insects have been documented and discussed by a number of authors including Ross (1965), Hennig (1969, 1981), Kristensen (1975, 1981, 1991, 1995, 1999), Boudreaux (1979), Rohdendorf \& Rasnitsyn (1980), Whiting et al. (1997), Whiting (1998), Wheeler et al. (2001) and Beutel \& Gorb (2001). An exhaustive review of the evidence is beyond the scope of this paper. Here we will discuss briefly the following clades, which are generally recognized in recent publications but not supported by our wing data, namely Hymenoptera + Mecopterida, Coleoptera + Neuropterida and Strepsiptera + Mecopterida: Antliophora.

Hymenoptera + Mecopterida is usually supported by the following apparent synapomorphies: (1) sitophore plate present on the base of the adult hypopharynx; (2) arolium present; (3) larval pretarsus unpaired; (4) larval silk-producing labial glands; (5) eruciform larva. The presence of a sitophore plate, discussed in detail by Vilhelmsen (1996) and Kristensen (1999), apprently facilitated the development of specialized sucking mouthparts in various groups of Hymenoptera and Mecopterida. A similar condition is also known in Hemineoptera, but this is considered homoplasious. The presence of an arolium was considered to be a synapomorphy by Beutel \& Gorb (2001), but this structure also occurs in Blattoneoptera and Hemineoptera, as well as in some Neuroptera. The unpaired larval pretarsus is a relatively weak, regressive character, while the larval labial silk gland also occurs in Psocoptera. The eruciform larva, with hypognathous head and abdominal prolegs, was suggested as a possible synapomorphy by Königsmann (1976), but Kristensen (1999) noted that larvae of Nannochoristidae (Mecoptera) and primitive Amphiesmenoptera are prognathous.

In an analysis by Vilhelmsen (2001) of 38 hymenopteran exemplars and six outgroups using 220 adult and 16 larval characters, a strict consensus of eight equally parsimonious trees consisted of three unresolved clades: Coleoptera, Hymenoptera and Mecopterida + Neuropterida, but the last one was not very strongly supported.

Neuropterida + Coleopterida is usually supported by (1) absence of cruciate cervicocoxal muscles; (2) reduced ovipositor with first valvulae reduced and fused, second valvulae not discrete and cerci not articulated; (3) head of 1Ax distinctly enlarged and cranially truncate; (4) caudal process of 2Ax present; and (5) tarsomeres with hairy adhesive soles. The first character is based on Chadwick's (1959) observations that the spinasternal musculature of larval Dytiscidae (highly reduced and of no diagnostic value in adult beetles) is similar to that in Megaloptera, and that Coleoptera, Megaloptera and Neuroptera lack the cruciate cervicocoxal muscles (Icv-cx1X), which are found in both Mecoptera and Hymenoptera. This reduction based on relatively small sample can provide only weak support for this clade. The loss of articulated cerci and reduction of the ovipositor are two independent characters, which were used in the scenario presented by Mickoleit (1973). The plesiomorphic, articulated cerci of Mecopterida and Hymenoptera are represented by fixed lobes on the ectoproct of Neuropterida, but the total absence of cerci in Coleoptera could be an independent loss. Similarly, the highly reduced ovipositor in Mecopterida could be as easily derived from the base of a Mecopterida + Neuropterida + Coleopterida lineage as it could from an ancestor of Hymenoptera (as shown in Mickoleit's cladogram). The third and fourth of these synapomorphies are taken from Hörnschemeyer (1998, 2002). Our comparative data on $1 \mathrm{Ax}$ in Coleoptera are limited, but one of us (JKP) conducted a broad study of $1 \mathrm{Ax}$ in the Neoptera orders. The 1Ax (especially the body) was found to be highly variable in shape and size and the plesiomorphic state in Neoptera could not be reconstructed with confidence and thus was not used in the order-level system proposed by Haas \& Kukalová-Peck (2001). The 2Ax body in Neuropterida is very short and without an elongated caudal projection (Fig. 34), and in Megaloptera it is connected with the distal corner of the $3 \mathrm{Ax}$ goblet by an extra long and strong ligament (Haas \& Kukalová-Peck, 2001, Fig. 17). But, in Coleoptera and Strepsiptera, the $2 \mathrm{Ax}$ body is posteriorly elongated into a more or less narrow caudal projection, which articulates directly with the median plate and there is no visible ligament (Figs 29, 30, 31; Kukalová-Peck \& Lawrence, 1993, Figs $75,77,78,80,81)$. The presence of hairy, adhesive tarsal soles was used by Beutel \& Gorb (2001) as a Neuropterida + Coleopterida synapomorphy; however this is highly variable within Coleoptera, as well as being present in Dermaptera and Embioptera. The fact that adhesive soles occur in many beetle species does not necessarily mean that they are part of the coleopteran groundplan. Tarsal pads are lacking in archostematan 
families Ommatidae, Micromalthidae and Crowsoniellidae, as well as in most Adephaga (excluding the protarsi of some males), all Myxophaga, and in many basal Polyphaga, including Derodontidae.

The morphological evidence for placing the order Strepsiptera within the Mecopterida: Antliophora has been most recently discussed by Whiting et al. (1997), Whiting (1998), Kristensen (1999), and Wheeler et al. (2001). As pointed out by Whiting (1998), a number of characters used to define Mecopterida and Antliophora must be coded as inapplicable in Strepsiptera due to the extreme reduction or loss of certain organs; included among these are all characters involving details of the maxilla, labium or ovipositor. Characters supporting an inclusion of Strepsiptera within Mecopterida (1-2) and Antliophora (3-6) include: (1) vestigial ovipositor; (2) absence of the outer pterothoracic tergo-coxal rotator muscle; (3) dagger-like mandible with anterior articulation reduced or lost; (4) prelabium without endite lobes and associated musculature; (5) male segment 9 enlarged and ring-like; (6) sperm pump. All other characters used by Whiting (1998) for this node were coded inapplicable for Strepsiptera. Of these, (1) represents an extreme reduction and is not very useful, and (2) and (4) are also reductions. The dagger-like mandibles, a character first noted by Mickoleit (1971), is questionable because of its occurrence in xyelid Hymenoptera (Kristensen, 1999). The ring-like segment 9 in the male was confused with a fusion of the gonopod bases, which are lacking in Strepsiptera. The transformation of segment 9 into a ring-like structure to which segment 10 is dorsally attached is, according to Kristensen (1999), a widespread feature with Mecopterida. It is also a common feature within Coleoptera, although perhaps not a part of the groundplan for the order. Kristensen also questioned whether the sperm pump of Strepsiptera is really homologous to those in Antliophora.

Phylogenies of the insect orders based on cladistic analyses of $18 \mathrm{~S}$ and $28 \mathrm{~S}$ ribosomal DNA nucleotide sequences have been produced by a number of authors, including Carmean et al. (1992), Pashley et al. (1993), Chalwatzis et al. (1996), Whiting et al. (1997), Whiting (1998), Wheeler et al. (2001) and Whiting (2002). Most of these cladograms support both Neuropterida + Coleoptera and Hymenoptera + Mecopterida, but the most remarkable result was the sister group relationship between Diptera and Strepsiptera in all $18 \mathrm{~S}$ analyses conducted not only by the New York lab (Whiting et al., 1997; Wheeler et al., 2001) but by another group in Darmstadt (Chalwatzis et al., 1996). Whiting \& Wheeler (1994) further proposed an origin of Strepsiptera involving a homoeotic mutation which reversed the mesothorax and metathorax. These results have been questioned by Carmean \& Crespi (1995), who argued that the apparently monophyletic Halteria was an artifact of longbranch attraction, and similar reservations were expressed by Huelsenbeck $(1997,1998,2001)$ and Hwang et al. (1998) with rebuttals by Whiting (1998), Siddall (1998) and Siddall \& Whiting (1999).
The most recent cladogram of the endoneopteran orders based on 18S ribosomal DNA data (Whiting, 2002) supports the monophyly of all orders except Coleoptera and Mecoptera. The latter is paraphyletic with respect to Siphonaptera, while Coleoptera is a parapyletic assemblage attached at four places in the tree. There are two main groups in Endoneoptera: Hymenoptera + Neuropterida + Mecoptera (including Siphonaptera) and Coleoptera + Amphiesmenoptera + Halteria (Strepsiptera and Diptera). The Coleoptera form two basal assemblages within the second group - Archostemata + Myxophaga and a monophyletic Polyphaga - but in addition the Hydradephaga and Omophron attach at the base of Amphiesmenoptera, while the Carabidae attach at the base of the Halteria. Whiting comments on possible causes of these results, noting that the great diversity of Coleoptera is an unlikely one considering that no such problem occurs for Lepidoptera or Hymenoptera. It seems that additional studies are required using different genes.

\section{Coleopteran suborders}

Phylogenetic relations proposed by Beutel \& Haas (1998, 2000). The hind wing evidence presented above for the beetle suborders does not support the phylogeny proposed by Beutel \& Haas (1998, 2000), and yet their cladogram is considered to be the "currently best supported" one, which is also "in good accordance with the reasonably good record of early beetle fossils" (Kristensen, 1999). It seems appropriate, then, to examine the characters used to support this alternate hypothesis, particularly those dealing with wing venation and folding. These authors coded 32 exemplar genera for 98 morphological characters. The taxa included 4 Neuropterida, 3 Archostemata representing Ommatidae, Cupedidae and Micromalthidae, 3 Myxophaga representing Torridincolidae, Sphaeriusidae and Hydroscaphidae, 9 Adephaga representing Gyrinidae, Haliplidae, Trachypachidae, Noteridae, Amphizoidae, Hygrobiidae, Dytiscidae and Carabidae and 13 Polyphaga representing Hydrophilidae, Hydraenidae, Agyrtidae, Leiodidae, Silphidae, Scarabaeidae, Byrrhidae, Elateridae, Cantharidae, Derodontidae, Coccinellidae, Tenebrionidae and Chrysomelidae. Of the 107 characters used in this analysis, 19 were based on larvae and one-third of the remaining ones were based on muscle losses or fusions in the pterothorax. The following comments will concentrate on those features used to support Adephaga + Myxophaga + Polyphaga or Myxophaga + Polyphaga, or to remove support from Archostemata + Adephaga + Myxophaga or Adephaga + Myxophaga.

B\&H Char. 30. Presternal cervical sclerites (autapomorphy of Polyphaga). This character relies on the argument that lateral cervical sclerites of presternal origin do not occur in endoneopteran outgroups and therefore must be an autapomorphy of Polyphaga. As pointed out by Lawrence (1999), Matsuda (1970) stated that lateral cervical sclerites of presternal origin occur in Corydalus and Chauliodes (Megaloptera) and Agulla (Raphidioptera), as well as in a variety of "hemimetabolous insects". It seems highly unlikely that this pair of hinged, musculated plates on each side of the neck has evolved de novo in 
polyphagan beetles, when similar structures are widespread throughout the insect orders. If they are present in beetle outgroups, then their presence in Polyphaga is plesiomorphic and their absence in Archostemata, Adephaga and Myxophaga is a synapomorphy. Also, an argument involving the origin of these cervical structures from presternum or preepisternum presupposes that the origin of the ventral sclerite in the coleopteran prothorax is well understood. It has been recently shown that in both mesothorax and metathorax, the "sternum" is a complex structure of pleural origin, the true sternum having been invaginated forming a forked endosternite (or paired endosternites) and externally marked by the discrimen (secondarily reduced in most mesothoraces). Since the prothorax is even more highly modified than the pterothoracic segments, how certain can we be that the proventrite contains any sternal component?

B\&H Char. 45. Ventrites of meso- and metathorax (both sclerites directly connected between and within mesocoxal cavities in Myxophaga and Polyphaga). As pointed out by Lawrence (1999), the support for this synapomorphy is weakened because the mesothorax and metathorax are joined by a membranous suture in most or all members of the polyphagan families Scirtidae, Decliniidae, Eucinetidae, Clambidae and Derodontidae, as well as some Agyrtidae (Apteroloma, Ipelates) and Staphylinidae (Anthobium, Megarthrus, Tachinus, etc.) (see below).

B\&H Char. 46. Katepisternal-mesocoxal joint (loss in Myxophaga, Adephaga and Polyphaga). This refers to the ventral condyle on the mesothorax, which was apparently homologous to those found in Mecoptera and Neuropterida. This structure, which was described and illustrated by Baehr (1975) for the archostematan genus Priacma, is also found in some basal Polyphaga (e.g. Stenocyphon and a few other Scirtidae) in which the mesoventral process is short and longitudinally divided by the discrimen, forming a pair of projections; it has been lost independently in Myxophaga + Adephaga (a synapomorphy) and in most Polyphaga.

B\&H Char. 47. Mesothoracic internalized discrimen (loss in Myxophaga, Adephaga and Polyphaga). An internalized discriminal line is present in various polyphagan groups, including Scirtidae, Buprestidae and Psephenidae. Its loss, like that of the katepisternal-mesocoxal joint, has occurred independently in Myxophaga + Adephaga and in Polyphaga.

B\&H Char. 78. Anterior margin of hind wing. This is a 4-state character concerning the anterior wing margin. It is said to be inflexible (state 0) in the outgroups; flexible but without a bending zone (1) in Omma and Priacma plus all non-staphyliniform Polyphaga; with a distinct bending zone (2) in Adephaga, Myxophaga, Hydrophilidae and Staphylinoidea; and with a marginal joint (3) in the Scarabaeoidea. No attempt is made to clearly define the bending zone. We do not agree with this coding. As discussed above, a region of one-way flexibility in the radial bar occurs not only in Adephaga and Staphyliniformia but also in Ommatidae and various non-staphyliniform polyphagans (Scirtoidea, Bostrichoidea). This condition appears to have been present in ancestral beetles, and was transformed into a sharp bend or hinge (radial hinge) in Cupedidae, Micromalthidae and independently in Myxophaga. The bending zone has been lost independently in various representatives of both polyphagan lineages as defined in our 1993 paper: the "hydrophiloid lineage" (Staphyliniformia including Scarabaeoidea) and the "eucinetoid lineage" (Scirtiformia, Elateriformia, Derodontiformia, Bostrichiformia and Cucujiformia). The marginal joint (state 3) refers to the apical hinge as defined in a previous section.

B\&H Char. 84. Proximo-ventral process on $2 \mathrm{Ax}$. This character, which refers to a proximal projection on the ventral side of $2 \mathrm{Ax}$ extending mesally beneath $1 \mathrm{Ax}$, was taken from Hörnschemeyer (1998, p. 68, character 23) and was referred to by both authors as a "lateral process". The presence of this feature in Adephaga, Myxophaga and Polyphaga is considered to be a synapomorphy and its absence in Archostemata a plesiomorphy. However, the projection also occurs in Ommatidae and is more likely to be a basal coleopteran condition, which was lost in Cupedidae and Micromalthidae. We have treated it as such in the above analysis (character 58).

With the removal of characters $46,47,78$ and 84 , the Adephaga + Myxophaga + Polyphaga clade in the Beutel \& Haas paper is still supported by 11 characters. The single larval character (19,1 - presence of urogomphi) is suspect, since it is highly unlikely that the various fixed or articulated processes on tergum 9 are homologous. They have probably evolved more than once in both Adephaga and Polyphaga. The highly reduced and concealed metatrochantin $(72,1)$ appears to be a good synapomorphy, but it does represent a reduction which could have occurred in more than one lineage. Little can be said about character 36 (reduction or loss of the spinasternum in Pantophaga), since the states are not clearly defined and the nature of the "spinasternum" in Coleoptera is complex and not well understood (Larsén, 1966). The remaining synapomorphies are all muscle losses or fusions, four from the mesothorax and four from the metathorax. Muscle characters are particularly difficult to deal with because of the special preparation necessary to study them (limiting the sample available for comparison) and the expertise and technology often necessary for proper observation. Character 94 (loss of the M. nototrochantinalis III) is obviously strongly correlated with the reduction of the metatrochantin (character 72). Characters 55 and 56, which refer to the loss of large mesothoracic tergopleural muscles, are correlated with the fusion of the meso- and metathorax in Adephaga (externally) and Myxophaga and Polyphaga (internally and often externally). However, it has been pointed out by Lawrence (1999) that this fusion is lacking in a number of basal Polyphaga for which thoracic muscles have not been described.

The Myxophaga + Polyphaga clade is supported by five characters in the Beutel \& Haas study. Three of these $(12,1$ : larval tibia fused with tarsus; 13,1 : larval claws sin- 
gle; and 34,1: protrochantin fused with pleuron) appear to be synapomorphies, although fusions and losses could represent parallel events correlated with size reduction. Character 45 was rejected above because of the presence of the plesiomorphic state in basal Polyphaga. The presence of an adult mandibular mola $(26,1)$ in Myxophaga and most basal Polyphaga was considered a synapomorphy based on the absence of a "true" mola in Neuropterida, as well as in Archostemata and Adephaga. Certainly, the presence of a basal mandibular processing area, with associated maxillary, epipharyngeal, pharyngeal and hypopharyngeal structures, occur in both Myxophaga and Polyphaga and are associated with a microphagous type of feeding (Lawrence, 1989, 1991) in which small particles, often suspended in liquid, are condensed into solid packets for passage into the gut. But is this a synapomorphy of Myxophaga and Polyphaga or a basal feature of the order Coleoptera? The loss of the adult mola may have occurred independently in Adephaga and Archostemata for two different reasons. Archostematan adults appear to be short lived, have reduced mandibles and probably take in water and/or nectar only; their wood-boring larvae, on the other hand, have evolved large mandibles with robust basal molae. Most Adephaga are predaceous as both larvae and adults and have developed heavy-bodied carnassial mandibles and maxillae. It is also possible that a primitive mandibular mola present in basal Neoptera may have been retained in endoneopteran ancestors and in basal Coleoptera, although lost in most other endoneopteran adults.

Phylogenetic relations proposed and documented here. The Archostemata + Adephaga + Myxophaga clade proposed by us in 1993 is well supported by synapomorphies involving the hind wing complex, which has been evaluated with equivalent criteria in all pterygote higher taxa. To support this clade convincingly with features from other, not fully homologized character complexes, is much more difficult. One such feature is the shared derived loss of lateral cervical sclerites in Archostemata, Adephaga and Myxophaga (see B\&H Char. 30 above). The clade is also united by two possible synapomorphies involving the male abdominal apex. In basal Polyphaga (Staphyliniformia, Scirtoidea, Elateriformia and Derodontoidea), males retain a well-developed tenth tergite, which is articulated to tergite 9, and a phallobase to which the parameres are attached. In all Archostemata, Myxophaga and Adephaga, the $10^{\text {th }}$ tergite has fused to the $9^{\text {th }}$, which is usually reduced and membranous, so that only the sclerotized subgenital plate or hypandrium remains. A separate phallobase is absent in all Myxophaga and almost all Adephaga and Archostemata; however, what appears to be a separate phallobase occurs in Tetraphalerus wagneri Waterhouse, and a remnant, more or less fused to the bases of the parameres, perists in at least some Gyrinidae (Spanglerogyrus, Macrogyrus). If tergite 10 and the free phallobase are considered to be basal coleopteran features, then they have been lost in most Archostemata and in Adephaga and Myxophaga. If the polyphagan phallobase is considered to be a subordinal autapomorphy, then it has independently arisen in basal Adephaga and Archostemata and then been lost again in both groups, which is less parsimonious.

Coleopteran suborders in fossil record. Beutel \& Haas (2000) claimed that the sister-group relationship between Archostemata and the remaining Coleoptera is more in accord with the fossil record than that proposed in our 1993 paper, and a similar statement was made by Kristensen (1999). These comments are based on the alleged record of "Cupedidae" from the Lower Permian $($ Sakmarian $=$ Asselian $)$, while the earliest polyphagan fossils are known from the Upper Triassic. If the first record were reliable (but see below), the conclusion would still be based on three theoretically unacceptable assumptions: (i) that all fossil Coleopterida with primitive elytra (containing large, seriate window-punctures) must be cupedids, (ii) that Coleoptera retaining primitive elytra must also have primitive hind wings, and (iii) that the earliest fossil record of a higher taxon must represent its first true occurrence. However, Ponomarenko (1995) pointed out that the Asselian fossil is probably not a beetle. The Tshekardocoleidae from the next oldest strata, Middle Lower Permian (Artinskian) and Upper Lower Permian (Kungurian), were placed by Crowson (1975) in a separate suborder Protocoleoptera, which was elevated to ordinal rank by Kukalová-Peck (1991) and expanded to include other Permian taxa such as Taldycupedidae, Asiocoleidae, Permocupedidae, and Rhombocoleidae. The group is considered to be a paraphyletic coleopteran stem assemblage. Although Tshekardocoleidae and some of the later Permian families have the plesiomorphic, large, linearly arranged, elytral window punctures found also in modern Cupedidae, they differ from beetles in having 13-segmented antennae, a shortened abdomen not closely coapted to the elytra, hind wings which do not fold apically (transversely), and an external ovipositor. An overlooked but highly relevant fact is that the oldest Upper Permian cupedid-like fossils (Permocupedidae) share the same deposits with about 12 genera and 35 other fossil species, the elytra of which bear no resemblance to any present day cupedid. These fossils are included in two families, Schizocoleidae and Permosynidae, which dominate the Lower Tartarian assemblages but are often ignored, since they were considered by Ponomarenko (1969) to be parataxonomic groupings. Schizocoleid elytra have a distinct longitudinal cleft (an internal ridge) resembling that of the Mesozoic families Schizophoridae and Catiniidae, while permosynid elytra are striate and similar to those of the family Ademosynidae. Although Ponomarenko (1969, 1995, 2002) defined Archostemata very broadly to include virtually all fossil beetles lacking the derived features of Adephaga or Polyphaga, he suggested that the ancestors of both Adephaga and Myxophaga might be among the schizophoroid families (Schizophoridae and Catiniidae), while Ademosynidae may well have included the ancestors of Polyphaga. Crowson (1975) and Kukalová-Peck (1991) restricted the suborder Archostemata to those taxa occurring primarily in the Mesozoic and Cenozoic, and Law- 
rence (1999) elevated Ponomarenko's family-group taxa, recognizing Triadocupedidae, Cupedidae and Ommatidae, all beginning in the Lower Triassic.

The problem with recognizing early Polyphaga is that the hind wing is almost never preserved and the fusion of propleuron and trochantin would be impossible to see in most fossil specimens. The presence of an external propleuron is insufficient to eliminate a fossil from Polyphaga, since it is usually indistinguishable from a polyphagan trochantinopleuron, which may be extensive in certain basal polyphagan groups. The problem is exacerbated by the small size of these early beetles. Based on elytral lengths, permosynids ranged in size from about 2 to $10 \mathrm{~mm}$ (Rohdendorf \& Ponomarenko, 1962).

A taxon of doubtful subordinal placement. Sikhotealinia zhiltzovae Lafer, an extant species from eastern Russia (Lafer, 1996) appears to be the only known beetle combining features of three suborders. The species was made the type of a new family but not placed in a suborder. It was moved by Kirejtshuk (1999) to the Jurodidae, an adephagan family based on Jurassic fossils (Ponomarenko, 1985), but transferred to the suborder Archostemata. Unfortunately, the specimen cannot be studied without visiting St. Petersburg, so conclusions concerning structural details are based on the author's illustrations and a few additional figures by Kirejtshuk (1999). The presence of what appears to be a well developed external propleuron extending behind the coxa, a metepisternum in broad contact with the mesocoxal cavity, and a slender metatrochantin (if it is indeed a separate sclerite) suggest a placement in Archostemata. The presence of six ventrites, the first of which lies partly beneath the metacoxae, appears to be an adephagan feature, but unlike the condition in Adephaga, the ventrites are free and the first one is not actually divided by the metacoxae. The wing venation is undoubtedly polyphagan, in spite of what appears to be a miniature, incomplete "oblongum" cell formed near the meeting of RP and MP1+2. The radial cell is of the polyphagan type, formed by a loop between RA1+2 and RA3 +4 , cross-vein $\mathrm{r} 3$ is almost horizontal, and MP1+2 is not sharply bent before the end of the medial bar in the folded wing. Perhaps a more complete study of this interesting species will shed more light on subordinal relationships.

Molecular phylogenetic evidence. It remains to mention briefly the results of recent works based on $18 \mathrm{~S}$ ribosomal DNA sequence data in Coleoptera. The tendency for Coleoptera to be shown as a non-monophyletic group has been mentioned in the previous section. Maddison et al. (1999) sequenced a large number of adephagan taxa (mainly Carabidae) plus Hydroscapha, four Polyphaga (Staphylinidae, Scarabaeidae, Clambidae, Tenebrionidae), and four Neuropterida (Sialidae, Raphidiidae, Ithonidae, Chrysopidae) and produced a cladogram in which Myxophaga and Adephaga are sister groups. Caterino et al. (2002) sampled a wide range of insect groups including one Archostemata (Cupedidae), three Myxophaga (Hydroscaphidae, Microsporidae, Torridincolidae), nine Adephaga (Haliplidae, Gyrinidae, Noteridae,
Hygrobiidae, Amphizoidae, Dytiscidae, Trachypachidae, Carabidae) and 12 Polyphaga (Hydrophilidae, Hydraenidae, Leiodidae, Silphidae, Scarabaeidae, Eucinetidae, Byrrhidae, Elateridae, Derodontidae, Coccinellidae, Tenebrionidae, Chrysomelidae). After constraining Coleoptera and its suborders to be monophyletic, a cladogram was produced in which Adephaga and Polyphaga were sister groups, with Myxophaga and Archostemata as basal lineages. Similar results were produced by Shull et al. (2001). To date none of the DNA studies have supported a basal split between Polyphaga and the remaining Coleoptera.

Thorax: structural integrity and phylogenetics. There is no doubt that modern Archostemata retain a large number of primitive attributes, which were present in the beetle ancestor. Most of these involve the retention of freely articulated thoracic sclerites and the muscles for moving or anchoring them. Members of this suborder lack some of the basic improvements present in most other beetle groups. Foremost among these is the increase in the structural integrity of the thorax, which involves the fusion or loss of a number of sclerites (fusion of the notum and sternum in front of the procoxae, reduction of the protrochantin or its fusion to the propleuron, fusion of the meso- and metathorax internally and/or externally, fusion of katepisternum and preepisternum and loss of the discrimen in the meso- and metathorax, and reduction and internalization of the metatrochantin), as well as the loss or partial fusion of various thoracic muscles. The question of whether or not these changes occurred once in an ancestor of Adephaga + Myxophaga + Polyphaga or more than once in Polyphaga and in Myxophaga is what separates our subordinal hypothesis from that of Beutel \& Haas (2000). The fusion of meso- and metathorax in Adephaga involves the meeting and interlocking of the mesoventrite and metaventrite while retaining the membranous meso-metathoracic joint within the coxal cavities. This same condition in Polyphaga occurs in Decliniidae, Eucinetidae and a few Scirtidae, but the extent of the meso-metaventral interlocking is never as great as in Adephaga. In Myxophaga and most Polyphaga the membranous joint is either replaced by a solid joint or completely lost. It seems likely then that the consolidation of meso- and metathorax has occurred at least three times during the early evolution of beetles.

ACKNOWLEDGEMENTS. We are grateful to the numerous institutions and individuals who have provided beetle specimens for wing dissections, not only for the 1993 study but for more recent works by JFL (see Acknowledgments in Lawrence et al., 1999). In addition, specimens of Hymenoptera were provided by M. J. Sharkey and G.A.P. Gibson, Neuropterida by L. Lambkin, Mecoptera and Trichoptera by O. Flint, and Lepidoptera by J. Rawlings. Portions of this work were completed with the assistance of operating grants from the Natural Sciences and Engineering Research Council of Canada (to JKP) and with facilities provided by CSIRO Entomology, Canberra (to JFL). R. Beutel, F. Haas, M. Horak, J.K. Liebherr, A.F. Newton, Jr., D.C.F. Rentz, S.A. Ślipiński, M.K. Thayer and O. Flint, are gratefully acknowledged for providing information and literature and for discussions on wing venation and folding. Special thanks are 
given to Marc Beurteaux of Toronto, Canada for his assistance in the production of the color figures, to Christine Lambkin for running a PAUP analysis based on a previous matrix, and to Joyce Cook for additional computing assistance. R. Beutel, F. Haas, R.A.B. Leschen, S.B. Peck, S.A. Ślipiński, P. Štys, P. Švácha, R.J. Wootton and two anonymous reviewers are thankfully acknowledged for reading and commenting on earlier versions of the manuscript.

\section{REFERENCES}

AbouheIf E. \& Wray G.A. 2002: Evolution of the gene network underlying wing polyphenism in ants. Science 297: 249-252.

Averof M. \& CoHen M. 1997: Evolutionary origin of insect wings from ancestral gills. Nature 385: 627-630.

Baehr M. 1975: Skelett und Muskulatur des Thorax von Priacma serrata LeConte (Coleoptera, Cupedidae). Z. Morphol. Tiere 81: 55-101.

Beutel M. \& GoRB S.N. 2001: Ultrastructure of attachment specializations of hexapods (Arthropoda): evolutionary patterns inferred from a revised ordinal phylogeny. J. Zool. Syst. Evol. Res. 39: 177-207.

Beutel R. \& HaAs F. 1998: Verwandtschaftsbeziehungen der Unterordnungen der Coleoptera (Insecta). In: Systematik im Aufbruch. 1. Jahrestagung der Gesellschaft für Biologische Systematik e. $V$. Zoologisches Forschungsinstitut und Museum Alexander König, Bonn, pp. 22-23.

Beutel R. \& HaAs F. 2000: Phylogenetic relationships of the suborders of Coleoptera (Insecta). Cladistics 16: 103-141.

Bocharova-Messner O.M. 1959: Development of the wing in the early postembryonic stages in the ontogeny of dragonflies (order Odonata). Trudy Inst. Morfol. Zhivot. Im. Severtsova 27: 187-200 [in Russian].

Boudreaux H.B. 1979: Arthropod Phylogeny, with Special Reference to Insects. John Wiley \& Sons, New York, viii +320 pp.

BRodSKy A.K: 1994. The Evolution of Insect Flight. Oxford University Press, Oxford, 229 pp.

Büning J. 1998: The ovariole: structure, type and phylogeny. In: Harrison F.W. \& Locke H. (eds): Microscopic Anatomy of Invertebrates 11C, Insecta. Wiley-Liss, New York, pp. 897-932.

Büning J. \& Maddison D.R. 1998: Surprising ovary structures at the base of Coleoptera. In: Brunnhofer V. \& Soldán T. (eds): Book of Abstracts, VIth European Congress of Entomology. Institute of Entomology, České Budějovice, p. 182.

Carmean D. \& Crespi B.J. 1995: Do long branches attract flies? Nature 373: 666.

Carmean D., Kimsey L.S. \& Berbee M.L. 1992: 18S rDNA sequences and holometabolous insects. Mol. Phylogenet. Evol. 1: 270-278.

CARPENTER F.M. 1992: Treatise on Invertebrate Palaeontology, Part R, Arthropoda 4. The Geological Society of America, Boulder, Colorado, $655 \mathrm{pp}$.

Caterino M.S., Shull V.L., Hammond P.M. \& Vogler A.P 2002: Basal relationships of Coleoptera inferred from $18 \mathrm{~S}$ rDNA sequences. Zool. Scripta 31: 41-49.

CHADwick L.E. 1959: Spinasternal musculature in certain insect orders. Smiths. Misc. Collect. 137: 117-156.

Chalwatzis N., Hauf J., van de Peer Y., Kinzelbach R. \& ZimMERMANN F.K. 1996: 18S ribosomal RNA genes of insects: primary structure of the genes and molecular phylogeny of the Holometabola. Ann. Entomol. Soc. Am. 89: 788-803.

Crowson R.A. 1955: The Natural Classification of the Families of Coleoptera. Nathaniel Lloyd, London, $187 \mathrm{pp}$.
Crowson R.A. 1960. The phylogeny of Coleoptera. Annu. Rev. Entomol. 5: 111-134.

CROwSON R.A. 1975: The evolutionary history of Coleoptera, as documented by fossil and comparative evidence. Atti del Congresso Nazionale Italiano di Entomologia, Sassari - 20-25 Maggio 1974, Firenze, Italy, pp. 47-90.

Forbes W.T.M. 1922: The wing-venation of the Coleoptera. Ann. Entomol. Soc. Am. 15: 328-345, pls. xxiv-xxv.

García-Bellido A.P. 1975: Genetic control of wing disc development in Drosophila. In: Cell Patterning. Ciba Foundation Symposium No. 29, pp. 161-182.

GoloBoff P.A. 1999: NONA ver. 2.0. P.A. Goloboff, Tucumán, Argentina.

HaAs F. \& Beutel R.G: 2001. Wing folding and the functional morphology of the wing base in Coleoptera. Zoology 104: 123-141.

HaAs F. \& KuKalovÁ-PecK J. 2001: Dermaptera hindwing structure and folding: new evidence for familial, ordinal and superordinal relationships within Neoptera (Insecta). Eur. J. Entomol. 98: 445-509.

HaAs F. \& Wootton R.J. 1996: Two basic mechanisms in insect wing folding. Proc. R. Entomol. Soc. London 263: 1651-1658.

HaAs F., Gorb S. \& Blickhan R. 2000: The function of resilin in beetle wings. Proc. R. Entomol. Soc. London 267: $1375-1381$.

Hamilton K.G.A. 1972: The insect wing, part II. Vein homology and the archetypal insect wing. J. Kansas Entomol. Soc. 45: 54-58.

HenNIG W. 1969: Die Stammesgeschichte der Insekten. Waldemar Kramer, Frankfurt am Main, 436 pp.

Hennig W. 1981: Insect Phylogeny. John Wiley, New York, 514 pp.

HöRnSCHEMEYER T. 1998: Morphologie und Evolution des Flügelgelenks der Coleoptera und Neuropterida. Bonner Zool. Monogr. 43: 1-126.

HÖRNSCHEMEYER T. 2002: Phylogenetic significance of the wingbase of the Holometabola (Insecta). Zool. Scripta 31: 17-29.

HuelsenBeCK J.P. 1997: Is the Felstenstein zone a fly trap? Syst. Biol. 46: 69-74.

HUELSENBECK J.P. 1998: Systematic bias in phylogenetic analysis: is the Strepsiptera problem solved? System. Biol. 47: 519-537.

HUELSENBECK J.P: 2001: A Bayesian perspective on the Strepsiptera problem. Tijdschr. Entomol. 144: 165-178.

Hwang U.W., Kim W., Tautz D. \& Friedrich M. 1998: Molecular phylogenetics at the Felsenstein Zone: approaching the Strepsiptera problem using 5.85 and $28 \mathrm{~S}$ rDNA sequences. Mol. Phylogenet. Evol. 9: 470-480.

KinZelBACH R.K. 1971: Morphologische Befunden an Fächerflügeln und ihre phylogenetische Bedeutung (Insecta: Strepsiptera). Zoologica 41: 1-256.

KinzelbaCh R.K. 1990: The systematic position of Strepsiptera (Insecta). Am. Entomol. 36: 292-303.

KIREJTSHUK A.G. 1999: Sikhotealinia zhiltzovae (Lafer, 1996) recent representative of the Jurassic coleopterous fauna (Coleoptera, Archostemata, Jurodidae). Proc. Zool. Inst. Russian Acad. Sci. 281: 21-26.

Klausnitzer B. 1975: Probleme der Abgrenzung von Unterordnungen bei den Coleoptera. Entomol. Abh. Staatl. Mus. Tierkd. Dresden 40: 269-275.

Königsmann E. 1976: Das phylogenetische System der Hymenoptera I. Dt. Entomol. Z. (N.F.) 23: 253-279. 
Kristensen N.P. 1975: The phylogeny of hexapod "orders". A critical review of recent accounts. Z. Zool. Syst. Evolutionsforsch. 13: $1-44$.

Kristensen N.P. 1981: Phylogeny of insect orders. Annu. Rev. Entomol. 26: 135-157.

KRISTENSEN N.P. 1991: Phylogeny of extant hexapods. In: CSIRO (ed.): The Insects of Australia. A Textbook for Students and Research Workers. Second Edition, Vol. 1. Melbourne University Press, Carlton, pp. 125-140.

KRISTENSEN N.P. 1995: Forty years' insects phylogenetic systematics. Zool. Beitr. (N. F.) 36: 83-124.

KRISTENSEN N.P. 1997: The groundplan and basal diversification of the hexapods. In: Fortey R.A. \& Thomas R.H. (eds): Arthropod Relationships. Chapman \& Hall, London, pp. 281-293.

KRISTENSEN N.P. 1999: Phylogeny of endopterygote insects, the most successful lineage of living organisms. Eur. J. Entomol. 96: $237-253$.

KuKALOVÁ-PeCK J. 1978: Origin and evolution of insect wings and their relation to metamorphosis, as documented by the fossil record. J. Morphol. 156: 53-126.

KuKalovÁ-Peck J. 1983: Origin of the insect wing and wing articulation from the arthropodan leg. Can. J. Zool. 61: $1618-1669$

KukalovÁ-Peck J. 1987: New Carboniferous Diplura, Monura, and Thysanura, the hexapod ground plan, and the role of the thoracic side lobes in the origin of wings (Insecta). Can. J. Zool. 65: 2237-2345.

KuKalovÁ-Peck J. 1991: Fossil history and the evolution of hexapod structures. In: C.S.I.R.O. (ed.): Insects of Australia. A Textbook for Students and Research Workers. Second Edition, Vol. 1. Melbourne University Press, Carlton, pp. 141-179.

KuKalovÁ-Peck J. 1997: Arthropod phylogeny and "basal" morphological structures. In: Fortey R.A. \& Thomas R.H. (eds): Arthropod Relationships. Chapman \& Hall, London, pp. 249-268.

Kukalová-Peck J. \& Brauckmann C. 1990: Wing folding in pterygote insects, and the oldest Diaphanopterodea from the early Late Carboniferous of West Germany. Can. J. Zool. 68 1104-1111.

KuKalová-Peck J. \& Lawrence J.F. 1993: Evolution of the hind wing in Coleoptera. Can. J. Zool. 125: 181-258.

Kukalová-Peck J. \& Richardson E.S. 1983: New Homoiopteridae (Insecta: Paleodictyoptera) with wing articulation from the Upper Carboniferous strata of Mazon Creek, Illinois. Can J. Zool. 61: 1670-1687.

LAFER G.SH. 1996: Family Sikhotealiniidae. In: Ler P.A. (ed.) Key to the Insects of the Russian Far East. Vol. III, Part 3. Dal'nauka, Vladivostok, pp. 390-396.

LARSÉN O. 1966: On the morphology and function of the locomotor organs of the Gyrinidae and other Coleoptera. Opusc. Entomol. (Suppl.) 30: 1-242

LAWRENCE J.F. 1989: Mycophagy in the Coleoptera: feeding strategies and morphological adaptations. In: Wilding N., Collins M., Hammond P.M. \& Webber J.F. (eds): InsectFungus Interactions. R. Entomol. Soc. Symp. Ser. 14, pp. $1-23$.

LAWRENCE J.F. 1991: Order Coleoptera.. In: Stehr F.W. (ed.) Immature Insects. Vol. II. Kendall-Hunt, Dubuque, Iowa, pp. 144-298.

LAWRENCE J.F. 1999: The Australian Ommatidae (Coleoptera: Archostemata): new species, larva and discussion of relationships. Invertebr. Taxon. 13: 369-390.

LAWRENCE J.F. 2001: A new genus of Valdivian Scirtidae (Coleoptera) with comments on Scirtoidea and the beetle suborders.
In: Morimoto K. et al. (eds): Sukunahikona. Special Publication of the Japan Coleopterological Society No. 1. Japan Coleopterological Society, Osaka, pp. 351-361.

Lawrence J.F. \& Newton A.F. Jr. 1995: Families and subfamilies of Coleoptera (with selected genera, notes and references, and data on family-group names). In: Pakaluk J. \& Ślipiński S.A. (eds): Biology, Phylogeny, and Classification of Coleoptera: Papers Celebrating the 80th Birthday of Roy A. Crowson. Muzeum i Instytut Zoologii, Polska Akademia Nauk, Warsaw, pp. 779-1006.

LaWRence J.F., Ślipiński S.A. \& PAKaluK J. 1995: From Latreille to Crowson: a history of the higher-level classification of beetles. In: Pakaluk J. \& Ślipiński S.A. (eds): Biology, Phylogeny, and Classification of Coleoptera: Papers Celebrating the 80th Birthday of Roy A. Crowson. Muzeum i Instytut Zoologii, Polska Akademia Nauk, Warsaw, pp. 87-154.

Lawrence J.F., Hastings A.M., Dallwitz M.J., Paine T.A. \& ZURCHER E.J. 1999: Beetles of the World: a Key and Information System for Families and Subfamilies. CD-ROM, version 1.0 for MS-Windows. C.S.I.R.O. Publishing, East Melbourne.

LESCHEN R.A.B. 2003: Erotylidae (Insecta: Coleoptera: Cucujoidea): phylogeny and review. Fauna of New Zealand 47: $1-108$.

Maddison D.R., BaKer M.D. \& Ober K.A. 1999: Phylogeny of carabid beetles as inferred from $18 \mathrm{~S}$ ribosomal DNA. Syst. Entomol. 24: 103-138.

Manton S.M. 1997: The Arthropoda. Clarendon Press, Oxford, $527 \mathrm{pp}$.

MartyNov A.V. 1923: On two basic types of insect wings and their significance for the general classification of insects. In: Deryugina K.M. (ed.): Trudy Perv. Vserossij. S'ezda Zool., Anatomov i Gistologov 1: 88-89.

Matsuda R. 1970: Morphology and evolution of the insect thorax. Mem. Entomol. Soc. Can. 76: 1-431.

Mickoleit G. 1971: Das Exoskelett von Notiothauma reedi McLachlan, ein Beitrag zur Morphologie und Phylogenie der Mecoptera (Insecta). Z. Morphol. Tiere 69: 318-362.

Mickoleit G. 1973: Über den Ovipositor der Neuropteroidea und Coleoptera und seine phylogenetische Bedeutung (Insecta, Holometabola). Z. Morphol. Tiere 76: 145-171.

Nixon K.C. 1999: Winclada (BETA) ver. 0.9.9. K.C. Nixon, Ithaca, New York.

Pashley D.P., McPheron B.A. \& Zimmer E.A. 1993: Systematics of holometabolous insect orders based on $18 \mathrm{~S}$ ribosomal RNA. Mol. Phylogenet. Evol. 2: 132-142.

PonOMARENKo A.G. 1969: The historical development of archostematan beetles. Trudy Paleontol. Inst. Akad. Nauk SSSR 125: 1-238 [in Russian].

PonOMARENKo A.G. 1985: Beetles from the Jurassic of Siberia and West Mongolia. In: Rasnitsyn A.P. (ed.): Jurassic Insects from Siberia and West Mongolia. Trudy Paleontol. Inst. Akad. Nauk SSSR 211: 47-87 [In Russian].

Ponomarenko A.G. 1995: The geological history of beetles. In: Pakaluk J. \& Ślipiński S.A. (eds): Biology, Phylogeny, and Classification of Coleoptera: Papers Celebrating the 80th Birthday of Roy A. Crowson. Muzeum i Instytut Zoologii, Polska Akademia Nauk, Warsaw, pp. 155-171.

PonomarenKo A. G. 2002: 2.2.1.3.2. Superorder Scarabaeidea Laicharting, 1781. Order Coleoptera Linné, 1858. The Beetles. In: Rasnitsyn A.P. \& Quicke D.L.J. (eds.): History of Insects. Kluwer Academic Publishers, Dordrecht, pp. $164-176$.

RoHDENDORF B.B. \& PonOMARENKo A.G. 1962: Order Coleoptera. Beetles. In: Rohdendorf B.B. (ed.): Fundamentals of Paleontology. Volume 9. Arthropoda, Tracheata, Chelicer- 
ata. Akademia Nauk SSSR, Moscow, pp. 241-267 [in Russian; translation 1991, D.R. Davis (ed.), Smithsonian Institution and National Science Foundation, Washington, D.C.].

RoHDENDORF B.B. \& RASNitsyn A.P. (eds) 1980: Historical development of the class Insecta. Trudy Paleontol. Inst. Akad. Nauk SSSR 175: 1-199.

Ross H.H. 1965: A Textbook of Entomology. $3^{\text {rd }}$ Edition. John Wiley \& Sons, New York, ix +539 pp.

SchNeIDER P. 1978: Die Flug- und Faltungstypen der Käfer (Coleoptera). Zool. Jb. Anat. Ontog. Tiere 99: 174-210.

Sharov A.G. 1966. Basic Arthropodan Stock. Pergamon Press, Elmsford, N.Y., $271 \mathrm{pp}$.

Shear W.A. \& KuKalová-Peck J. 1990: The ecology of Paleozoic terrestrial arthropods: the fossil evidence. Can. J. Zool. 68: $1807-1834$.

Shubin N., Tabin C. \& Carroll S. 1997. Fossils, genes and the evolution of animal limbs. Nature 388: 639-648.

Shull V.L., Vogler A.P., BaKer M.D., Maddison D.R. \& HamMOND P.M. 2001: Sequence alignment of 18S ribosomal RNA and the basal relationships of adephagan beetles: evidence for monophyly of aquatic families and the placement of Trachypachidae. Syst. Biol. 50: 945-969.

SIDDALL M.E. 1998: Success of parsimony in the four-taxon case: long-branch repulsion by likelihood in the Farris zone. Cladistics 14: 209-220.

SidDAll M.E. \& Whiting M.F. 1999: Long-branch abstractions. Cladistics 15: 9-24.

SNODGRASS R.E. 1935: Principles of Insect Morphology. McGraw-Hill, New York, $\mathrm{x}+667$ pp.

STENZHORN H.J. 1974: Experimentelle Untersuchungen zur Entwicklung des Lymantria dispar L. (Lepidoptera). Wilhelm Roux Arch. EntwMech. Org. 175: 65-86.

Šulc K. 1927: Das Tracheensystem von Lepisma (Thysanura) und Phylogenie der Pterygogenea. Acta Soc. Sci. Nat. Moravicae 4(7), $108 \mathrm{pp}$

TOWER W.L. 1903: The origin and development of the wings of Coleoptera. Zool. Jb. Anat. 17: 517-572.

VILHELMSEN L. 1996: The preoral cavity of lower Hymenoptera (Insecta): comparative morphology and phylogenetic significance. Zool. Scripta 25: 143-170.

VilHeLmsen L. 2001: Phylogeny and classification of the extant basal lineages of the Hymenoptera (Insecta). Zool. J. Linn. Soc. 131: 393-442.

Wallace F.L. \& Fox R.C. 1980: A comparative morphological study of the hindwing venation of the order Coleoptera, Part II. Proc. Entomol. Soc. Wash. 82: 609-654.

WARD R.D. 1979: Metathoracic wing structures as phylogenetic indicators in the Adephaga (Coleoptera). In: Erwin T.L., Ball G.E., Whitehead D.R. \& Halpern A.L. (eds): Carabid Beetles: Their Evolution, Natural History, and Classification. W. Junk, The Hague, pp. 113-180.

Wheeler W.C., Whiting M., Wheeler Q.D. \& Carpenter J.M. 2001: The phylogeny of the extant hexapod orders. Cladistics 17: 113-169.

Whiting M.F. 1998: Phylogenetic position of the Strepsiptera: review of molecular and morphological evidence. Int. J. Insect Morphol. Embryol. 27: 53-60.

Whiting M.F. 2002: Phylogeny of the holometabolous insect orders: molecular evidence. Zool. Scripta 31: 3-15.

Whiting M.F. \& Kathirithamby J. 1995: Strepsiptera do not share hind wing venational synapomorphies with Coleoptera: a reply to Kukalová-Peck and Lawrence. J. N. Y. Entomol. Soc. 103: 1-14

Whiting M.F. \& WheELER W.C. 1994: Insect homeotic transformation. Nature 368: 696.
Whiting M.F., Carpenter J.C., Wheeler Q.D. \& Wheeler W.C. 1997: The Strepsiptera problem: phylogeny of the holometabolous insect orders inferred from $18 \mathrm{~S}$ and $28 \mathrm{~S}$ ribosomal DNA sequences and morphology. Syst. Biol. 46: 1-68.

WiLlmann R. 1994: Raphidiodea aus dem Lias und die Phylogenie der Kamelhalsfliegen (Insecta: Holometabola). Palaeontol. Z. 68: 167-197.

WILLMANN R. 1997: Advances and problems in insect phylogeny. In: Fortey R.A. \& Thomas R.H. (eds): Arthropod Relationships. Chapman \& Hall, London, pp. 269-279.

Wоoтton R.J. 1976: The fossil record and insect flight. In: Rainey R.C. (ed.): Insect Flight. R. Entomol. Soc. London Symp. Ser. 7, pp. 235-254.

Wootton R.J. 1979: Function, homology and terminology in insect wings. Syst. Entomol. 4: 81-93.

Wоoтton R.J. 1981: Support and deformability in insect wings. J. Zool. (London) 193: 447-468.

Wootton R.J. 1990: The mechanical design of insect wings. Sci. Am. 262: 114-120.

Wootton R.J. 1992: Functional morphology of insect wings. Annu. Rev. Entomol. 37: 113-140.

Wootton R.J. 1995: Geometry and mechanics of insect hind wing fans: a modelling approach. Proc. R. Soc. London (B) 262: 181-187.

WootTon R.J. 1996: Functional wing morphology in Hemiptera systematics. In: Schaefer C.W. (ed.): Studies on Hemipteran Phylogeny. Entomological Society of America, Lanham, Maryland, pp. 179-198.

Wootton R.J. \& Kukalová-Peck J. 2000: Flight adaptation in Palaeozoic Palaeoptera (Insecta). Biol. Rev. 75: 129-167.

Zherikhin V.V. \& Gratshev V.G. 1995: A comparative study of the hind wing venation of the superfamily Curculionoidea, with phylogenetic implications. In: Pakaluk J. \& Ślipiński S.A. (eds): Biology, Phylogeny, and Classification of Coleoptera: Papers Celebrating the 80th Birthday of Roy A. Crowson. Muzeum i Instytut Zoologii, Polska Akademia Nauk, Warsaw, pp. 633-777.

\section{APPENDIX 1. PARTIAL LISTS OF GENERA EXAMINED}

In non-Coleoptera taxa, only those are listed which are documented by large detailed figures of wings and pteralia currently in the files of JKP. Many additional specimens have been studied. All modern taxa were compared with numerous specimens from fossil stem groups documented by figures. Both files are available to interested readers should a need for additional documentation arise.

\section{PLECONEOPTERA}

\section{PLECOPTERA}

\section{ARCTOPERLARIA}

Pteronarcyidae: Pteronarcella Banks 1900, Pteronarcys Newmann 1838

Perlodidae: Dictyogenus Klapálek 1904, Isoperla Banks 1906, Megarcys Klapálek 1912, Perlodes Banks 1903

Perlidae: Dinocras Klapálek 1907, Doroneuria Needham \& Claassen 1922, Oyamia Klapálek 1907, Paragnetina Klapálek 1907

Chloroperlidae: Siphonoperla Zwick 1967

Taeniopterygidae: Brachyptera Newport 1848, Doddsia Needham \& Claassen 1925, Taenionema Bolivar 1906, Taeniopteryx Janse 1920

Nemouridae: Amphinemura Ris 1902, Nemoura Latreille 1796, Nemurella Kempny 1898, Protonemura Kempny 1898

Leuctridae: Leuctra Stephens 1833, Pachyleuctra Despax 1920, Perlomyia Banks 1906 
Capniidae: Capnia Pictet 1841, Utacapnia Nebeker \& Gaulin 1967

ANTARCTOPERLARIA:

Eustheniidae: Cosmioperla McLellan 1996, Eusthenia Westwood 1838, Stenoperla M'Lachlan 1866, Thaumatoperla Tillyard 1921

Diamphipnoidae: Diamphipnopsis Illies 1960

Gripopterygidae: Dinotoperla Tillyard 1921, Trinotoperla Tillyard 1924

Austroperlidae: Austroheptura Illies 1969

\section{ORTHONEOPTERA}

\section{CAELIFERA}

Acrididae: Aiolopus Fieber 1781, Austroicetes Uvarov 1921, Caloptenopsis Bolivar 1889, Gastrimargus Saussure 1884, Gesonula Stål 1878, Goniaea Stål 1873, Heteropternis Stål 1873, Hippiscus Saussure, 1861, Choristocetes Brunner 1893, Locusta Linnaeus 1758, Pseudaiolopus Hollis 1967 , Pycnodictya Stål 1873, Pycnostictus Saussure 1884, Qualetta Sjöstedt 1921, Schistocerca Stål 1873, Sphingonotus Fieber 1852, Valanga Uvarov 1923

Eumastacidae: Biroella Bolivar 1903, Erucius Stål 1875 , Oedaleus Fieber 1853, Paramastax Burr 1899, Taeniopoda Stål 1879, Tytthotyle Scudder 1897, Trimerotrophis Stål 1873

Pamphagidae: Hoplolopha Stål 1876, Eremopeza Saussure 1888, Lobosceliana Dirsch 1958

Pneumoridae: Physemacris Roberts 1941

Pyrgomorphidae: Desmoptera Bolivar 1894, Desmopterella Ramme 1941, Petasida White 1845, Phymateus Thunberg 1815

Romaleidae: Eutropidacris Hebard 1923

Tetrigidae: Scelimena Audinet-Serville 1839

Tridactylidae: Rhipipteryx Audinet-Serville 1839

Trigonopterygidae: Trigonopteryx Charpentier 1841

\section{ENSIFERA}

Haglidae: Cyphoderria Uhler 1864

Gryllidae: Acheta Fabricius 1770, Brachytrupes AudinetServille 1839, Gryllus Linnaeus 1758, Teleogryllus Chopard 1961

Schizodactylidae: Schizodactylus Brullé 1835

Gryllotalpidae: Gryllotalpa Latreille 1802, Scapteriscus Scudder 1872

Tettigoniidae: Anabrus Haldeman 1852, Banza Walker 1870, Capnobotes Scudder 1872, Clonia Stål 1855, Metrioptera Wesmael 1838, Neobarrettia Rehn 1901, Neoconocephalus Karny 1907, Phyllophorella Karny 1929, Scudderia Stål 1873, Tympanophora White 1841

Stenopelmatidae: Gryllotaurus Karny 1929, Genus? (Heniciinae), Schizodactylus Blanchard 1845

Gryllacrididae: Bothriogryllacris Rentz 1993, Gryllacris Audinet-Serville 1831, Hadrogryllacris Karny 1937, Xanthogryllacris Karny 1937

Cooloolidae: Cooloola Rentz 1980

\section{PHASMATODEA}

Phylliidae: Chitoniscus Stål 1875, Phyllium Illiger 1798

Phasmatidae: Acontiometriotes Audinet-Serville 1838, Acrophylla Gray 1835, Cotylosoma Wood-Mason 1878, Eurycnema Audinet-Serville 1838, Palophus Westwood 1859, Prisopus Lepeletier \& Audinet-Serville 1828

Pseudophasmatidae: Stratocleus Stål 1875

\section{BLATTONEOPTERA}

\section{DERMAPTERA}

Diplatyidae: Diplatys Audinet-Serville 1831, Haplodiplatys Hincks 1955
Pygidicranidae: Pyragra Audinet-Serville 1831, Echinosoma Audinet-Serville 1838, Tagalina Dohrn 1863

Anisolabididae: Carcinophora Scudder 1876

Labiduridae: Allostethus Verhoeff 1904, Forcipula Bolivar 1898, Labidura Rafinesque 1815, Nala Zacher 1910

Apachyidae: Apachyus Audinet-Serville 1831

Spongiophoridae: Labia Leach 1815, Marava Burr 1911, Sparatta Audinet-Serville 1831, Spongiphora AudinetServille 1831, Vostox Burr 1911

Forficulidae: Allodahlia Verhoeff 1902, Ancistrogaster Stål 1855, Forficula Linnaeus 1758

Chelisochidae: Chelisoches Scudder 1876, Chelisochella Verhoeff 1902

\section{MANTODEA}

Mantoididae: Mantoida Newman 1838

Choeradolidae: Choeradodis Audinet-Serville 1831

Mantidae: Hierodula Burmeister 1838, Rhombodera Burmeister 1838, Mantis Linnaeus 1758, Macromantis Saussure 1871

Hymenopodidae: Pseudocreobotra Saussure 1870, Genus?

Empusidae: Empusa Illiger 1798

Chaeteessidae: Chaeteessa Burmeister 1838

\section{ISOPTERA}

Mastotermitidae: Mastotermes Froggatt 1897

Termopsidae: Porotermes Hagen 1858, Stolotermes Hagen 1858, Zootermopsis Emerson 1933

Hodotermitidae: Hodotermes Hagen 1853, Microhodotermes Sjöstedt 1926

Kalotermitidae: Neotermes Holmgren 1911

BLATTODEA

Blattidae: Eurycotis Stål 1874, Periplaneta Burmeister 1838 Polyphagidae: Polyphaga Brullé 1835

Blattellidae: Neotemnopteryx Princis 1951

Blaberidae: Calolampra Saussure 1893, Leucophaea Brunner v. Wattenwyl 1865, Panesthia Audinet-Serville 1831

\section{HEMINEOPTERA}

\section{CICADOMORPHA}

Tettigarctidae: Tettigarcta White 1845

Cercopidae: Leptotaspis??, Mahanarva Distant 1909, Tomaspis Amyot \& Audinet-Serville 1843

\section{FULGOROMORPHA}

Eurybrachidae: Eurybrachys Guérin-Méneville 1833, Platybrachys Stål 1859

Fulgoridae: Enchophora Spinola 1839, Phrictus Koch 1873

Copidocephalidae: Copidocephala Stål 1869

Cixiidae: Paranagnia Hampson 1926

Lophopidae: Genus?

Flatidae: Ityraea Stål 1866, Siphanta Stål 1860

Ricaniidae: Ricania Germar 1818

Achilidae: Achilus Kirby 1819

HETEROPTERA

Belostomatidae: Lethocerus Mayr 1853

\section{ENDONEOPTERA}

\section{HYMENOPTERA}

Xyelidae: Pleroneura Konow 1897, Macroxyela Kirby 1882, Xyela Dalman 1819

Tenthrediniidae: Trimex Linnaeus 1763

Pamphiliidae: Pamphilius Latreille 1802, Genus?

Pergidae: Perga Leach 1817, Philomastix Froggatt 1890

Sphecidae: Genus?

Argidae: Runaria Malaise 1931

Megalodontidae: Tristactoides Chevin 1985

Braconidae: Helcon Nees 1812 


\section{NEUROPTERIDA \\ NEUROPTERA}

Ithonidae: Varnia Walker 1860, Ithone Newman 1838, Megaithone Riek 1974, Oliarces Banks 1908

Rapismatidae: Rapisma M'Lachlan 1866

Dilaridae: Nallachius Navás 1909, Genus?

Coniopterygidae: Neosemidalis Enderlein 1930, Spiloconis Enderlein 1907

Berothidae: Proberotha Krueger 1923, Protobiella Tillyard 1923, Spermophorella Tillyard 1916

Mantispidae: Ditaxis M'Lachlan 1867, Campion Navás 1914

Sisyridae: Sisyra Burmeister 1839

Neurorthidae: Austroneurorthus Nakahara 1958

Psychopsidae: Psychopsis Newman 1842, Megapsychops Tillyard 1919, Genus?

Polystoechotidae: Polystoechotes Burmeister 1839, Fontecilla Navás 1832

Osmylidae: Oedosmylus Krueger 1913, Eidoporismus Krueger 1916

Hemerobiidae: Drepanacra Tillyard 1916, Notherobius New 1988, Psychobiella Banks 1909, Zachobiella Banks 1920

Chrysopidae: Ankylopteryx Brauer 1864, Dictyochrysa Esben-Petersen 1917, Italochrysa Principi 1946, Oligochrysa Esben-Petersen 1914, Triplochrysa Kimmins 1952

Nymphidae: Myiodactylus Brauer 1866, Norfolius Navás 1922, Nymphes Leach 1814

Nemopteridae: Chasmatoptera Berthold 1827

Myrmeleontidae: Distoleon Banks 1910, Palpares Rambur 1842, Periclystus Gerstaecker 1888, Weeleus Navás 1912

\section{MEGALOPTERA}

Corydalidae: Archichauliodes Van der Weele 1909, Chauliodes Oken 1816, Neohermes Banks 1908, Neoneuromus Van der Weele 1909, Neurhermes Navás 1915, Nigronia Banks 1908, Protochauliodes Van der Weele 1909, Protohermes Van der Weele 1907

Sialidae: Sialis Latreille 1802, Stenosialis Tillyard 1919, Austrosialis Tillyard 1919

\section{RAPHIDIOPTERA}

Raphidiidae: Agulla Navás 1914

Inocelliidae: Parainocellia Aspöck \& Aspöck 1968, Inocellia Schneider 1843

\section{MECOPTERIDA}

MECOPTERA

Notiothaumidae: Notiothauma M'Lachlan 1877

Nannochoristidae: Nannochorista Tillyard 1917

Panorpidae: Panorpa Linnaeus 1758

Choristidae: Choristis Bonaparte 1854, Taeniochorista Esben-Petersen 1914

\section{DIPTERA}

Tipulidae: Holorusia Loew 1863, Prolimnophila Alexander 1929

Tabanidae: Tabanus Linnaeus 1758

Asilidae: Diogmites Loew 1866

TRICHOPTERA

Philopotamidae: Dolophilodes Ulmer 1909

Polycentropodidae: Polycentropus Curtis 1835

Limnephilidae: Clistoronia Banks 1916, Limnophilus Fitzinger 1843

Rhyacophilidae: Rhyacophila Pictet 1834

Phryganeidae: Phryganea Linnaeus 1758

Hydropsychidae: Baliomorpha Neboiss 1984, Hydropsyche Kelaart 1858

Calamoceratidae: Anisocentropus M'Lachlan 1863

\section{LEPIDOPTERA}

Hepialidae: Fraus Walker 1856, Trictena Meyrick 1932

Neopseustidae: Neopseustis Meyrick 1932

Agaristidae: Hecatesia Boisduval 1828

\section{COLEOPTERIDA}

STREPSIPTERA

Mengenillidae: Mengenilla Hofeneder 1910

Corioxenidae: Triozocera Pierce 1911

Halictophagidae: Coriophagus Kinzelbach 1971

Myrmecolacidae: Caenocholax Pierce 1909, Lychnocolax Bohart 1951

\section{COLEOPTERA}

\section{ARCHOSTEMATA}

Ommatidae: Omma Newman 1839, Tetraphalerus Waterhouse 1901

Micromalthidae: Micromalthus LeConte 1879

Cupedidae: Adinolepis Neboiss 1984, Cupes Fabricius 1801, Distocupes Neboiss 1984, Priacma LeConte 1874, Prolixocupes Neboiss 1960, Tenomerga Neboiss 1984

\section{MYXOPHAGA}

Lepiceridae: Lepicerus Motschulsky 1855

Torridincolidae: Claudiella Reichardt \& Vanin 1976, Iapir Py-Daniel et al. 1993, Satonius Endrödy-Younga 1997, Torridincola Steffan 1964, Ytu Reichardt 1973

Hydroscaphidae: Hydroscapha LeConte 1874

Sphaeriusidae: Sphaerius Waltl 1838

\section{ADEPHAGA}

Gyrinidae: Macrogyrus Régimbart 1883, Spanglerogyrus Folkerts 1979

Haliplidae: Haliplus Latreille 1802

Trachypachidae: Systolosoma Solier 1849, Trachypachus Motschulsky 1844

Noteridae: Hydrocanthus Say 1823, Notomicrus Sharp 1882

Amphizoidae: Amphizoa LeConte 1854

Hygrobiidae: Hygrobia Latreille 1804

Dytiscidae: Copelatus Erichson 1832, Eretes Laporte 1833, Hyderodes Hope 1838, Hyphydrus Illiger 1807, Laccophilus Leach 1817, Lancetes Sharp 1882, Megaporus Brinck 1943

Rhysodidae: Omoglymmius Ganglbauer 1892

Carabidae: Adelotopus Hope 1834, Amarotypus Bates 1872 , Arthropterus Macleay 1838, Calosoma Weber 1801, Catadromus Macleay 1825, Cicindela Linnaeus 1758, Elaphrus Fabricius 1775, Gehringia Darlington 1933, Hiletus Schiödte 1847, Loricera Latreille 1802, Mecyclothorax Sharp 1903, Megacephala Latreille 1802, Mormolyce Hagenbach 1825, Nebria Latreille 1806, Omophron Latreille 1802, Opisthius Kirby 1837, Ozaena Olivier 1812, Paussus Linnaeus 1775, Pheropsophus Solier 1833, Scarites Fabricius 1775

POLYPHAGA

Hydrophiloidea

Hydrophilidae: Amphiops Erichson 1843, Coelostoma Brullé 1835, Dactylosternum Wollaston 1854, Epimetopus Lacordaire 1854, Georissus Latreille 1809, Helobata Bergroth 1888, Helophorus Fabricius 1775, Hydrochus Leach 1817, Hydrophilus Geoffroy 1762, Limnoxenus Motschulsky 1853, Pseudohydrobius Blackburn 1898, Rygmodus White 1846, Spercheus Kugelann 1798, Sperchopsis LeConte 1861, Sternolophus Solier 1834

Sphaeritidae: Sphaerites Duftschmid 1805

Synteliidae: Syntelia Westwood 1864

Histeridae: Hololepta Paykull 1811, Niponius Lewis 1885, Pactolinus Motschulsky 1860, Phelister Marseul 1853, 
Platysoma Leach 1817, Teretriosoma Horn 1873, Trypanaeus Eschscholtz 1829, Trypeticus Marseul 1864

Staphylinoidea

Hydraenidae: Hydraena Kugelann 1794, Limnebius Leach 1915, Parhydraenida Balfour-Browne 1975, Ochthebius Leach 1815, Tympanogaster Janssens 1967

Ptiliidae: Acrotrichis Motschulsky 1848, Nossidium Erichson 1845

Agyrtidae: Agyrtes Frölich 1799, Apteroloma Hatch 1927, Ipelates Reitter 1884, Necrophilus Latreille 1829, Pteroloma Gyllenhal 1827, Zeanecrophilus Newton 1997

Leiodidae: Anisotoma Panzer 1797, Colon Herbst 1797, Dietta Sharp 1876, Eublackburniella Jeannel 1937, Hydnobius Schmidt 1847, Myrmecholeva Lea 1910, Platycholeus Horn 1880, Zeadolopus Broun 1903

Scydmaenidae: Horaeomorphus Schaufuss 1889

Silphidae: Diamesus Hope 1840, Nicrophorus Fabricius 1775, Ptomaphila Hope 1840

Staphylinidae: Apatetica Westwood 1848, Austrolophrum Steel 1938, Baeocera Erichson 1845, Brathinus LeConte 1852, Creophilus Leach 1819, Deinopteroloma Jansson 1946, Empelus LeConte 1861, Glypholoma Jeannel 1962, Kalissus LeConte 1874, Megalopinus Eichelbaum 1915, Micropeplus Latreille 1809, Microsilpha Broun 1886, Sagola Sharp 1874, Sartallus Sharp 1871, Scaphidium Olivier 1790, Scaphium Kirby 1837, Siagonium Kirby \& Spence 1815, Stenus Latreille 1796

\section{Scarabaeoidea}

Lucanidae: Aesalus Fabricius 1801, Ceratognathus Westwood 1838, Ceruchus Macleay 1819, Dorcus Macleay 1819, Echinoaesalus Zelenka 1993, Figulus Macleay 1819, Lamprima Latreille 1807, Lucanobium Howden \& Lawrence 1974, Mitophyllus Parry 1843, Nicagus LeConte 1860, Penichrolucanus Deyrolle 1863, Pholidotus Macleay 1819, Platycerus Geoffroy 1762, Rhyssonotus Macleay 1819, Sinodendron Hellwig 1894, Syndesus Macleay 1819

Passalidae: Aulacocyclus Kaup 1868, Leptaulax Kaup 1868

Trogidae: Omorgus Erichson 1847, Trox Fabricius 1775

Glaresidae: Glaresis Erichson 1848

Pleocomidae: Pleocoma LeConte 1856

Diphyllostomatidae: Diphyllostoma Fall 1901

Bolboceratidae: Australobolbus Howden \& Cooper 1977, Blackburnium Boucomont 1911, Elephastomus Macleay 1819, Eucanthus Westwood 1848

Geotrupidae: Frickius Germain 1897, Geotrupes Latreille 1796, Neoathyreus Howden \& Martinez 1963, Typhaeus Leach 1815

Ochodaeidae: Ochodaeus Dejean 1821

Ceratocanthidae: Ceratocanthus White 1842, Cyphopisthes Gestro 1899, Ivieolus Howden \& Gill 1988

Hybosoridae: Anaides Westwood 1841, Coilodes Westwood 1846, Cryptogenius Westwood 1846, Liparochrous Erichson 1848, Phaeochrous Laporte 1840

Glaphyridae: Amphicoma Latreille 1807, Cratoscelis Erichson 1835, Lichnanthe Burmeister 1844

Scarabaeidae: Acrossidius Schmidt 1914, Aegialia Latreille 1807, Allidiostoma Arrow 1940, Anomala Samouelle 1819 , Anoplognathus Leach 1815, Aulonocnemis Klug 1838, Chiron Macleay 1819, Copris Geoffroy 1762, Cryptodus Macleay 1819, Cyclocephala Dejean 1821, Dichelonyx Harris 1827, Dichotomius Hope 1838, Glycyphana Burmeister 1842, Haploscapanes Arrow 1908, Hoplia Illiger 1803, Onthophagus Latreille 1807, Orphnus Macleay 1819, Oryctomorphus Guérin-Méneville 1830, Osmoderma Le Peletier \& Audinet-Serville 1828, Pachypus Audinet-
Serville 1828, Phaenognatha Hope 1842, Phaenomeris Hope 1835, Phanaeus Macleay 1819, Phileurus Latreille 1807, Saprus Blackburn 1904, Serica Macleay 1819, Trichius Fabricius 1775, Valgus Scriba 1790, Xylotrupes Hope 1837

\section{Scirtoidea}

Decliniidae: Declinia Nikitsky et al. 1994

Eucinetidae: Eucinetus Germar 1818, Nycteus Latreille 1829 Clambidae: Acalyptomerus Crowson 1979, Calyptomerus Redtenbacher 1849, Clambus Fischer von Waldheim 1820, Sphaerothorax Endrödy-Younga 1959

Scirtidae: Amplectopus Sharp 1886, Atopida White 1846, Byrrhopsis Champion 1913, Cyphon Paykull 1799, Cyphotelus Sharp 1878, Elodes Latreille 1796, Macrocyphon Pic 1918, Macrodascillus Carter 1935, Macrohelodes Blackburn 1892, Microcara Thomson 1859, Ora Clark 1865, Prionocyphon Redtenbacher 1858, Sacodes LeConte 1853, Sarabandus Leech 1955, Scirtes Illiger 1807, Stenocyphon Lawrence 2001, Veronatus Sharp 1878

\section{Dascilloidea}

Dascillidae: Anorus LeConte 1859, Coptocera Murray 1868, Dascillus Latreille 1796, Genecerus Walker 1871, Karumia Escalera 1913, Notodascillus Carter 1935

Rhipiceridae: Rhipicera Latreille 1817, Sandalus Knoch 1801

Buprestoidea

Buprestidae: Acmaeodera Eschscholtz 1829, Agrilus Curtis 1825, Brachys Dejean 1833, Buprestis Linnaeus 1758, Cisseis Gory \& Laporte 1839, Coraebus Laporte \& Gory 1839, Cylindromorphus Théry 1930, Dystaxia LeConte 1866, Endelus Deyrolle 1864, Germarica Blackburn 1888, Glyptoscelimorpha Horn 1893, Helferella Cobos 1957, Julodimorpha Thomson 1878, Julodis Eschscholtz 1829, Nascio Gory \& Laporte 1837, Notobubastes Carter 1924, Paratrachys Saunders 1873, Polycesta Solier 1833, Schizopus LeConte 1858, Sternocera Eschscholtz 1829, Stigmodera Eschscholtz 1829, Thrincopyge LeConte 1858, Trachys Fabricius 1801, Xyroscelis Saunders 1868

\section{Byrrhoidea}

Byrrhidae: Byrrhus Linnaeus 1758, Cytilus Erichson 1847, Microchaetes Hope 1834, Morychus Erichson 1847

Elmidae: Lara LeConte 1852, Phanocerus Sharp 1882, Potamophilinus Grouvelle 1896, Simsonia Carter \& Zeck 1929, Stenelmis Dufour 1835, Stetholus Carter \& Zeck 1929

Dryopidae: Helichus Erichson 1847, Pelonomus Erichson 1847, Spalacosostea Kodada 1996

Lutrochidae: Lutrochus Erichson 1847

Limnichidae: Byrrhinus Motschulsky 1858, Ersachus Erichson 1847, Martinius Spilman 1959, Paralimnichus Delève 1973, Physemus LeConte 1854, Throscinus LeConte 1874

Heteroceridae: Heterocerus Fabricius 1792, Lanternarius Pacheco 1964, Micilus Mulsant \& Rey 1872

Psephenidae: Acneus Horn 1880, Dicranopselaphus GuérinMéneville 1861, Eubria Germar 1818, Eubrianax Kiesenwetter 1874, Psephenoides Gahan 1914, Psephenus Haldeman 1853, Sclerocyphon Blackburn 1892

Cneoglossidae: Cneoglossa Guérin-Méneville 1843

Ptilodactylidae: Aploglossa Guérin-Méneville 1849, Araeopidius Cockerell 1906, Austrolichas Lawrence \& Stribling 1992, Byrrocryptus Broun 1893, Cladotoma Westwood 1837, Daemon Laporte 1836, Drupeus Lewis 1895, Octoglossa Guérin-Méneville 1843, Paralichas White 1859, Ptilodactyla Illiger 1807, Therius Guérin-Méneville 1849 
Chelonariidae: Brounia Sharp 1878, Chelonarium Fabricius 1801, Pseudochelonarium Pic 1916

Eulichadidae: Eulichas Jakobson 1911, Stenocolus LeConte 1853

Callirhipidae: Callirhipis Latreille 1829, Celadonia Laporte 1840, Ennometes Pascoe 1866, Ptorthocera Champion 1896, Zenoa Say 1835

\section{Elateroidea}

Artematopodidae: Allopogonia Cockerell 1906, Artematopus Perty 1830, Electribius Crowson 1973, Eurypogon Motschulsky 1859, Macropogon Motschulsky 1859

Rhinorhipidae: Rhinorhipus Lawrence 1988

Brachypsectridae: Brachypsectra LeConte 1874

Cerophytidae: Cerophytum Latreille 1809

Anischiidae: Anischia Fleutiaux 1896

Eucnemidae: Anelastes Kirby 1818, Dyscharachthis Blackburn 1900, Fornax Laporte 1835, Galbites Fleutiaux 1918, Hemiopsida Macleay 1872, Oisocerus Bonvouloir in Murray 1868, Perothops Laporte 1838, Phyllocerus Le Peletier \& Audinet-Serville 1828, Schizophilus Bonvouloir 1870, Trigonopleurus Bonvouloir 1871

Throscidae: Aulonothroscus Horn 1890, Pactopus LeConte 1868, Potergus Bonvouloir 1871

Elateridae: Ampedus Dejean 1833, Athous Eschscholtz 1829, Austrelater Calder \& Lawrence 1993, Campsosternus Latreille 1834, Cebrio Olivier 1790, Conoderus Eschscholtz 1829, Cussolenis Fleutiaux 1918, Denticollis Piller \& Mitterpacher 1783, Drapetes Dejean 1821, Hemiops Laporte 1836, Melanotus Eschscholtz 1829, Negastrius Thomson 1859, Octinodes Candèze 1863, Oestodes LeConte 1853, Oxynopterus Hope 1842, Paracalais Neboiss 1967, Paracardiophorus Schwarz 1895, Physodactylus Fischer von Waldheim 1823, Pityobius LeConte 1853, Protelater Sharp 1877, Pseudotetralobus Schwarz 1902, Scaptolenus LeConte 1853, Semiotus Eschscholtz 1829

Plastoceridae: Plastocerus Schaum 1852

Drilidae: Drilus Olivier 1790, Selasia Laporte 1836

Omalisidae: Omalisus Geoffroy 1762

Lycidae: Calochromus Guérin-Méneville 1833, Celetes Newman 1838, Leptolycus Leng \& Mutchler 1922, Lycus Fabricius 1787, Lypropaeus Waterhouse 1878, Platerodrilus Pic 1921, Porrostoma Laporte 1838

Telegeusidae: Telegeusis Horn 1895

Phengodidae: Euryopa Gorham 1881, Phengodes Illiger 1807

Rhagophthalmidae: Diplocladon Gorham 1883, Dodecatoma Westwood 1843, Rhagophthalmus Motschulsky 1854

Lampyridae: Amydetes Illiger 1807, Cladodes Solier 1849, Cyphonocerus Kiesenwetter 1879, Drilaster Kiesenwetter 1879, Flabellotreta Pic 1911, Harmatelia Walker 1858, Lucidota Laporte 1833, Luciola Laporte 1833, Magnoculus McDermott 1964, Ototretadrilus Pic 1921, Photinus Laporte 1833, Photuris Laporte 1833, Psilocladus Blanchard 1846, Pterotus LeConte 1859, Pyractonema Solier 1849

Omethidae: Drilonius Kiesenwetter 1874, Matheteus LeConte 1874, Troglomethes Wittmer 1970

Cantharidae: Cantharis Linnaeus 1758, Chauliognathus Hentz 1830, Discodon Gorham 1881, Dysmorphocerus Solier 1849, Heteromastix Boheman 1858, Ichthyurus Westwood 1848, Malthodes Kiesenwetter 1852, Oontelus Solier 1849, Sphaerarthrum Waterhouse 1884, Tytthonyx LeConte 1851

\section{Derodontoidea}

Derodontidae: Derodontus LeConte 1861, Laricobius Rosenhauer 1846, Nothoderodontus Crowson 1959, Peltastica Mannerheim 1852

Jacobsoniidae: Derolathrus Sharp 1908, Saphophagus Sharp 1886, Sarothrias Grouvelle 1918

\section{Bostrichoidea}

Nosodendridae: Nosodendron Latreille 1804

Dermestidae: Adelaidia Blackburn 1891, Anthrenocerus Arrow 1915, Anthrenus Geoffroy 1762, Attagenus Latreille 1802, Dermestes Linnaeus 1758, Egidyella Reitter 1899, Evorinea Beal 1961, Mariouta Pic 1898, Neoanthrenus Armstrong 1941, Novelsis Casey 1900, Orphilus Erichson 1846, Rhopalosilpha Arrow 1921, Trichelodes Carter 1935, Trinodes Dejean 1821, Trogoderma Berthold 1827

Endecatomidae: Endecatomus Mellié 1847

Bostrichidae: Apoleon Gorham 1885, Bostrychopsis Lesne 1899, Chilenius Lesne 1921, Lichenophanes Lesne 1899, Lyctus Fabricius 1792, Melalgus Dejean 1835, Minthea Pascoe 1866, Psoa Herbst 1797, Rhyzopertha Stephens 1830, Stephanopachys Waterhouse 1888, Tetrapriocera Horn 1878, Tristaria Reitter 1878

Anobiidae: Anobium Fabricius 1775, Calymmaderus Solier 1849, Clada Pascoe 1887, Deroptilinus Lea 1924, Dryophilodes Blackburn 1891, Ernobius Thomson 1859, Fabrasia Martinez \& Viana 1954, Hadrobregmus Thomson 1859, Lasioderma Stephens 1835, Leanobium Español 1972, Ptilineurus Reitter 1901, Ptilinus Müller 1764, Secretipes Lea 1924, Trichodesma LeConte 1861, Xeranobium Fall 1905

Lymexyloidea

Lymexylidae: Atractocerus Palisot de Beauvois 1801, Elateroides Schaeffer 1766, Lymexylon Fabricius 1775, Melittomma Murray 1867

\section{Cleroidea}

Phloiophilidae: Phloiophilus Stephens 1830

Trogossitidae: Acalanthis Erichson 1844, Ancyrona Reitter 1876, Calitys Thomson 1859, Cylidrella Sharp 1891, Decamerus Solier 1849, Diontolobus Solier 1849, Egolia Erichson 1842, Eronyxa Reitter 1876, Grynocharis Thomson 1859, Larinotus Carter \& Zeck 1937, Lepidopteryx Hope 1840, Neaspis Pascoe 1872, Ostoma Laicharting 1781, Peltonyxa Reitter 1876, Protopeltis Crowson 1964, Temnoscheila Westwood 1830, Thymalus Latreille 1802

Chaetosomatidae: Chaetosoma Westwood 1851

Cleridae: Allochotes Westwood 1875, Crobenia Blackburn 1891, Cylidrus Latreille 1829, Epiclines Chevrolat 1838, Eunatalis Schenkling 1909, Ichnea Laporte 1836, Isoclerus Lewis 1892, Lecontella Wolcott \& Chapin 1918, Lemidia Spinola 1841, Necrobia Olivier 1795, Neichnea Wolcott \& Chapin 1918, Odontophlogistus Elston 1923, Pelonides Kuwert 1894, Sedlacekvia Winkler 1989, Tarsostenus Spinola 1844, Thaneroclerus Chapin 1924, Trogodendron Spinola 1841, Zenodosus Wolcott 1910

Acanthocnemidae: Acanthocnemus Perris 1866

Prionoceridae: Idgia Laporte 1836, Prionocerus Perty 1831

Mauroniscidae: Mauroniscus Bourgeois 1911, Scuromanius Majer 1995

Melyridae: Arthrobrachus Solier 1849, Astylus Laporte 1836, Carphurus Erichson 1840, Collops Erichson 1840, Cradytes Casey 1895, Dasyrhadalus Fall 1910, Dicranolaius Champion 1921, Enicopus Stephens 1830, Melyris Fabricius 1775, Melyrodes Gorham 1882, Rhadalus LeConte 1852, Semijulistus Schilsky 1894 


\section{Cucujoidea}

Protocucujidae: Ericmodes Reitter 1878

Sphindidae: Aspidiphorus Latreille 1829, Notosphindus McHugh \& Wheeler 1991, Odontosphindus LeConte 1878, Protosphindus Sen Gupta \& Crowson 1979, Sphindophorus Sen Gupta \& Crowson 1979, Sphindus Dejean 1821

Kateretidae: Amartus LeConte 1861, Anthonaeus Horn 1879

Nitidulidae: Aethina Erichson 1843, Brachypeplus Erichson 1842, Calonecrus Thomson 1857, Cryptarcha Shuckard 1839, Cybocephalus Erichson 1844, Cychramptodes Reitter 1878, Cychrocephalus Reitter 1873, Glischrochilus Reitter 1873, Maynipeplus Kirejtshuk 1998, Meligethes Stephens 1830, Mystrops Erichson 1843, Pallodes Erichson 1843, Pityophagus Shuckard 1839, Pocadius Erichson 1843, Thalycra Erichson 1843, Thalycrodes Blackburn 1891

Smicripidae: Smicrips LeConte 1878

Monotomidae: Lenax Sharp 1877, Monotoma Herbst 1793, Rhizophagus Herbst 1793, Shoguna Lewis 1884

Boganiidae: Athertonium Crowson 1990, Boganium Sen Gupta \& Crowson 1966, Paracucujus Sen Gupta \& Crowson 1966

Helotidae: Helota Macleay 1825

Phloeostichidae: Agapytho Broun 1921, Hymaea Pascoe 1869, Myrabolia Reitter 1876, Phloeostichus Crowson 1973, Priasilpha Broun 1893, Rhopalobrachium Boheman 1858, Tasmosalpingus Lea 1919

Silvanidae: Ahasverus Gozis 1881, Aplatamus Grouvelle 1912, Cryptamorpha Wollaston 1854, Monanus Sharp 1879, Platamus Erichson 1845, Psammoecus Latreille 1829, Silvanus Latreille 1804, Telephanus Erichson 1846, Uleiota Latreille 1796

Passandridae: Ancistria Erichson 1845, Passandra Dalman 1817, Taphroscelidia Crotch 1873

Cucujidae: Cucujus Fabricius 1775, Palaestes Perty 1830 , Pediacus Shuckard 1839, Platisus Erichson 1842

Laemophloeidae: Carinophloeus Lefkovitch 1961, Cryptolestes Ganglbauer 1899, Lathropus Erichson 1846, Laemophloeus Dejean 1836, Placonotus Macleay 1871

Propalticidae: Propalticus Sharp 1879

Phalacridae: Acylomus Sharp 1888, Litochrus Erichson 1845, Olibrus Erichson 1845, Phalacrinus Blackburn 1891, Phalacrus Paykull 1798, Tolyphus Erichson 1845

Cyclaxyridae: Cyclaxyra Broun 1893

Lamingtoniidae: Lamingtonium Sen Gupta \& Crowson 1969

Hobartiidae: Hobartius Sen Gupta \& Crowson 1966, Hydnobioides Sen Gupta \& Crowson 1966

Cavognathidae: Cavognatha Crowson 1964, Taphropiestes Reitter 1875

Cryptophagidae: Caenoscelis Thomson 1863, Catopochrotus Reitter 1889, Cryptogasterus Leschen 1996, Cryptophagus Herbst 1792, Hypocoprus Motschulsky 1839, Telmatophilus Heer 1841

Erotylidae: Anadastus Gorham 1887, Brachysphaenus Lacordaire 1842, Cathartocryptus Sharp 1886, Cnecosa Pascoe 1866, Cryptophilus Reitter 1874, Dacne Latreille 1796, Encaustes Lacordaire 1842, Episcaphula Crotch 1876, Hapalips Reitter 1877, Leucohimatium Rosenhauer 1856, Loberus LeConte 1861, Megalodacne Crotch 1873 , Microsternus Lewis 1887, Othniocryptus Sharp 1900 , Pharaxonotha Reitter 1875, Setariola Jakobson 1915, Thallis Erichson 1842, Thallisella Crotch 1876, Toramus Grouvelle 1916, Triplax Herbst 1793, Xenocryptus Arrow 1929
Byturidae: Byturus Latreille 1796, Dascillocyphon Everts 1909, Platydascillus Everts 1909, Xerasia Lewis 1895

Biphyllidae: Althaesia Pascoe 1860, Biphyllus Dejean 1821, Diplocoelus Guérin-Méneville 1838, Gonicoelus Sharp 1900

Bothrideridae: Ascetoderes Pope 1961, Bothrideres Erichson 1845, Craspedophilus Heinze 1943, Dastarcus Walker 1858, Deretaphrus Newman 1842, Erotylathris Motschulsky 1861, Oxylaemus Erichson 1845, Sosylus Erichson 1845, Sysolus Grouvelle 1908, Teredolaemus Sharp 1885, Teredomorphus Heinze 1943, Xylariophilus Pal \& Lawrence 1986

Cerylonidae: Cerylon Latreille 1802, Glyptolopus Erichson 1845, Hypodacnella Ślipiński 1988, Murmidius Leach 1822, Mychocerinus Ślipiński 1990, Ostomopsis Scott 1922

Discolomatidae: Discoloma Erichson 1845, Fallia Sharp 1902

Endomychidae: Eidoreus Sharp 1885, Encymon Gerstaecker 1857, Holoparamecus Curtis 1833, Idiophyes Blackburn 1895, Micropsephodes Champion 1913, Phymaphora Newman 1838, Stenotarsus Perty 1832, Trochoideus Westwood 1833

Coccinellidae: Diomus Mulsant 1850, Epilachna Dejean 1837, Harmonia Mulsant 1850, Rhyzobius Stepens 1829, Serangium Blackburn 1889, Stethorus Weise 1885, Sticholotis Crotch 1874

Corylophidae: Ectinocephalus Matthews 1888, Foadia Pakaluk 1985, Hoplicnema Matthews 1899, Orthoperus Stephens 1829, Periptyctus Blackburn 1895, Priamima Pakaluk \& Lawrence 1986

Latridiidae: Aridius Motschulsky 1866, Cortinicara Johnson 1975, Enicmus Thomson 1859

Tenebrionoidea

Mycetophagidae: Berginus Erichson 1846, Filicivora Leschen \& Lawrence 1991, Litargus Erichson 1846, Mycetophagus Hellwig 1792, Pseudochrodes Reitter 1876, Pseudotriphyllus Reitter 1879, Thrimolus Casey 1900, Triphyllus Dejean 1821

Archeocrypticidae: Archeocrypticus Kaszab 1964, Australenneboeus Kaszab 1984, Enneboeopsis Champion 1894, Enneboeus Waterhouse 1878, Nothenneboeus Lawrence 1994

Pterogeniidae: Histanocerus Motschulsky 1858, Pterogenius Candèze 1861

Ciidae: Cis Latreille 1796, Octotemnus Mellié 1847, Orthocis Casey 1898, Sphindocis Fall 1917, Xylographus Mellié 1847

Tetratomidae: Notopisenus Nikitsky \& Lawrence 1991, Penthe Newman 1838, Pisenus Casey 1900, Tetratoma Fabricius 1790, Triphyllia Reitter 1898

Melandryidae: Eustrophopsis Champion 1889, Hallomenus Panzer 1794, Melandrya Fabricius 1801, Orchesia Latreille 1807, Osphya Illiger 1807, Phloeotrya Stephens 1832, Zilora Mulsant 1856

Mordellidae: Hoshihananomia Kôno 1935

Rhipiphoridae: Eorhipidius Iablokov-Khnzorian 1986, Euctenia Gerstaecker 1855, Macrosiagon Hentz 1830, Metoecus Dejean 1834, Neonephrites Riek 1955, Nephrites Shuckard 1838, Pelecotoma Fischer 1809, Rhipidioides Riek 1955, Trigonodera Dejean 1834

Zopheridae: Aprostoma Guérin-Méneville, Aulonium Erichson 1845, Docalis Pascoe 1860, Ethelema Pascoe 1960, Hyporhagus Thomson 1860, Latometus Erichson 1842, Monoedus Horn 1882, Nematidium Erichson 1845, Noserinus Casey 1907, Orthocerodes Ślipiński \& Lawrence 
1999, Orthocerus Latreille 1796, Pristoderus Hope 1840, Pseudendestes Lawrence 1980, Pycnomerus Erichson 1842, Todima Grouvelle 1893

Ulodidae: Dipsaconia Pascoe 1860, Meryx Latreille 1807, Trachyderas Philippi 1864, Ulodes Erichson 1842

Perimylopidae: Promecheilus Solier 1851, Sirrhas Champion 1893

Chalcodryidae: Chalcodrya Redtenbacher 1868, Onysius Broun 1886

Trachelostenidae: Trachelostenus Solier 1851

Tenebrionidae: Acropteron Perty 1830, Adelium Kirby 1818, Bolitotherus Candèze 1861, Bothrotes Casey 1907, Chariotheca Pascoe 1860, Clamoris Gozis 1886, Cnemeplatia Costa 1847, Corticeus Piller \& Mitterpacher 1783, Cossyphus Olivier 1795, Cryphaeus Klug 1833, Cyphaleus Westwood 1841, Diaperis Müller 1764, Lagria Fabrcius 1775, Leaus Matthews \& Lawrence 1992, Leiochrinus Westwood 1883, Lepispilus Westwood 1841, Luprops Hope 1833, Nilio Latreille 1802, Omophlus Dejean 1834, Opatrum Fabricius 1775, Phaleria Latreille 1802, Phrenapates Kirby 1837, Statira Audinet-Serville 1828, Strongylium Kirby 1818, Tenebrio Linnaeus 1758, Trachyscelis Latreille 1809, Uloma Dejean 1821, Zolodinus Blanchard 1853

Prostomidae: Dryocora Pascoe 1868, Prostomis Latreille 1838

Synchroidae: Mallodrya Horn 1888, Synchroa Newman 1838

Oedemeridae: Agasma Newman 1850, Calopus Fabricius 1775, Chrysanthia Schmidt 1844, Nacerdes Falderman 1836, Pseudolycus Guérin-Méneville 1833, Sparedrus Latreille 1829, Thelyphassa Pascoe 1876

Stenotrachelidae: Anelpistus Horn 1870, Cephaloon Newman 1838, Nematoplus LeConte 1855, Stenotrachelus Latreille 1825, Stolius Lewis 1895

Meloidae: Anthicoxenus Fairmaire \& Germain 1860, Cerocoma Fabricius 1775, Deridea Westwood 1875, Eletica Dejean 1834, Epicauta Dejean 1834, Horia Fabricius 1787, Iselma Haag-Rutenberg 1879, Nemognatha Illiger 1807, Tetraonyx Latreille 1805

Mycteridae: Hemipeplus Latreille 1825, Lacconotopedilus Pic 1935, Lacconotus LeConte 1862, Madrasiindus Pic 1911, Mycterus Clairville 1798, Stilponotus Gray 1832

Boridae: Boros Herbst 1797, Lecontia Champion 1893, Synercticus Newman 1842

Trictenotomidae: Trictenotoma Gray 1832

Pythidae: Anaplopus Blackburn 1890, Ischyomius Chevrolat 1878, Priognathus LeConte 1850, Pytho Latreille 1796, Sphalma Horn 1888, Trimitomerus Horn 1888

Pyrochroidae: Agnathus Germar 1818, Cycloderus Solier 1851, Morpholycus Lea 1917, Pedilus Fischer 1822, Pyrochroa Geoffroy 1762, Temnopalpus Blackburn 1888, Tydessa Peacock 1982

Salpingidae: Elacatis Pascoe 1860, Inopeplus Smith 1851, Istrisia Lewis 1895, Neosalpingus Blackburn 1891, Ocholissa Pascoe 1863, Prostominia Reitter 1889, Rhinosimus Latreille 1805, Serrotibia Reitter 1877, Tretothorax Lea 1910

Anthicidae: Afremus Levey 1985, Anthicus Paykull 1798, Copobaenus Fairmaire \& Germain 1863, Duboisius Abdullah 1961, Egestria Pascoe 1871, Formicomus LaFerté-Senéctère 1848, Ictistygna Pascoe 1866, Lagrioida Fairmaire \& Germain 1860, Lemodes Boheman 1858, Macratria Newman 1838, Mecynotarsus LaFerté-Senéctère 1847, Mitraelabrus Solier 1851, Pseudotomoderus Pic
1892, Sapintus Casey 1895, Steropes Steven 1806, Trichananca Blackburn 1891

Aderidae: Elonus Casey 1895, Megaxenus Lawrence 1990, Scraptogetus Broun 1893, Syzeton Blackburn 1891, Syzetonellus Blackburn 1891, Syzetoninus Blackburn 1891, Zonantes Casey 1895

Scraptiidae: Allopoda LeConte 1866, Anaspis Geoffroy 1762, Canifa LeConte 1866, Diclidia LeConte 1862, Scraptia Latreille 1807

\section{Chrysomeloidea}

Oxypeltidae: Oxypeltus Blanchard 1851

Vesperidae: Paramigdolus Dias 1986, Philus Saunders 1853, Vesperus Latreille in Cuvier 1829, Vesperoctenus Bates 1891

Disteniidae: Cyrtonops White 1853, Distenia Le Peletier \& Audinet-Serville in Latreille 1828, Typodryas Thomson 1864

Cerambycidae: Apatophysis Chevrolat 1860, Archetypus Thomson 1860, Asemum Eschscholtz 1830, Atimia Haldeman 1847, Cerambyx Linnaeus 1758, Dorcasomus Audinet-Serville 1834, Enicodes Thomson 1860, Erichsonia Westwood 1849, Lamia Fabricius 1775, Leptura Linnaeus 1758, Lissonotus Dalman in Schönherr 1817, Mastododera Thomson 1864, Molorchus Fabricius 1792, Navomorpha Thomson 1860, Necydalis Linnaeus 1758, Parandra Latreille 1804, Phaolus Pascoe 1863, Psephactus Harold 1879, Pyrodes Audinet-Serville 1832, Rhagium Fabricius 1775, Sagridola Thomson 1864, Spondylis Fabricius 1775, Syllitus Pascoe 1859, Tapeina Audinet-Serville 1825, Thaumasus Reiche 1853

Megalopodidae: Colobaspis Fairmaire 1894, Cucujopsis Crowson 1946, Palophagus Kuschel 1990, Zeugophora Kunze 1818

Orsodacnidae: Aulacoscelis Duponchel \& Chevrolat 1842, Orsodacne Latreille 1802

Chrysomelidae: Altica Geoffroy 1762, Alurnus Fabricius 1775, Aspidimorpha Hope 1840, Brontispa Sharp 1904, Callosobruchus Pic 1902, Carpophagus Macleay 1826, Chlamisus Rafinesque 1815, Chrysochus Chevrolat 1837, Chrysophtharta Weise 1901, Clytra Laicharting 1781, Colaspis Fabricius 1801, Cryptocephalus Geoffroy 1762, Donacia Fabricius 1775, Hispellinus Weise 1897, Hornius Fairmaire 1885, Lamprolina Baly 1855, Lamprosoma Kirby 1818, Lema Fabricius 1798, Leptinotarsa Chevrolat 1836, Megascelis Sturm 1826, Microdonacia Blackburn 1893, Notosacantha Chevrolat 1837, Oides Weber 1801, Rhyparida Baly 1861, Sagra Fabricius 1792, Stethopachys Baly 1861, Syneta Dejean 1835, Uroplata Chevrolat 1837

\section{Curculionoidea}

Nemonychidae: Basilogeus Kuschel 1994, Brarus Kuschel 1997, Cimberis Gozis 1881, Lecontellus Kuschel 1989, Nemonyx Redtenbacher 1845

Anthribidae: Anthribus Geoffroy 1762, Araecerus Schönherr 1823, Euparius Schönherr 1823, Urodontellus Louw 1993

Belidae: Aralius Kuschel 1990, Arhinobelus Zimmerman 1994, Rhinotia Kirby 1819

Caridae: Car Blackburn 1897

Attelabidae: Auletobius Desbrochers 1869, Byctiscus Thomson 1859, Cycnotrachelus Jekel 1860, Eugnamptus Schoenherr 1839, Homoeolabus Jekel 1860, Merhynchites Sharp 1889, Pterocolus Say 1831

Brentidae: Ankleineela Zimmerman 1994, Antliarhis Billberg 1820, Apion Herbst 1797, Austronanodes Zimmerman 1993, Cylas Latreille 1802, Cyphagogus Parry 1849, Eurhynchus Kirby 1828, Ithycerus Schönherr 1823, 
Leoapion Zimmerman 1994, Rhinorhynchidius Voss 1922, Rhynolaccus Guérin-Méneville 1831, Tanaos Schönherr 1826, Tracheloschizus Damoiseau 1966, Ulocerus Schönherr 1823

Curculionidae: Amorphocerus Schönherr 1826, Aonychus Schönherr 1844, Araucarius Kuschel 1966, Austroplatypus Browne 1971, Chrysolopus Germar 1817, Cossonus Clairville 1798, Cryptoderma Ritsema 1885, Cyrtobagous Hustache 1929, Desmidophorus Dejean 1835, Dryophthorus Germar 1824, Hylurgops LeConte 1876, Neochetina Hustache 1926, Notoplatypus Lea 1909, Phaenomerus Schönherr 1836, Rhynchophorus Herbst 1795, Scolytoplatypus Schaufuss 1890, Tomicoproctus Faust 1898

\section{APPENDIX 2. KUKALOVÁ-PECK \& LAWRENCE (1993): CHANGES IN FIGURE LABELS}

In the past decade, one of us (JKP) researched the dual nature of the anojugal lobe in Neoptera and followed the characteristic veinal reductions that took place in the partial lobe of Blattoneoptera, Hemineoptera and Endoneoptera, and of the endoneopteran superorders (see also p. 97). This new insight brought a chain reaction of changes in veinal symbols, and a number of additional superordinal and order-level characters. The subsequent changes are required in the old figures of the hind wings of Coleoptera: $\mathrm{CuA}=\mathrm{Cu}, \mathrm{CuA} 1+2=\mathrm{CuA}, \mathrm{CuA} 3+4$ $=\mathrm{CuP}, \mathrm{CuA} 2=\mathrm{CuA} 3+4, \mathrm{AA}=\mathrm{AA} 3+4, \mathrm{AA} 1+2=\mathrm{AA} 3$, $\mathrm{AA} 3+4=\mathrm{AA} 4$. We devoted much of the time allotted to the reconstruction of higher-level groundplans to the most variable and difficult cubital area, by comparing left and right hind wings in many additional specimens. The more broadly verified and more precise nomenclature of the cubital branches is specified below.

Fig. 15. Spanglerogyrus. There are two sclerotizations shown in the region between MP1+2 and MP3; these are referred to as medial flecks (= binding patches). The smaller and more proximal of the two is an anomalous sclerotization found in a single wing only, while the second, larger one may represent a darkened area of wing membrane, rather than a binding patch, and could not be found in subsequent wing dissections. A true medial binding patch does not occur in Gyrinidae and when present in Adephaga, lies between MP3 and MP4 (as in KukalováPeck \& Lawrence, 1993, Fig. 19, Hydrocanthus, and Fig. 20, Trachypachus).

Fig. 30. Omma. The cross-vein at the apex of the wedge cell is $\mathrm{CuA} 3+4 . \mathrm{CuA} 2$ is not changed.

Fig. 31. Omma, apical region. A sharp hinge, labelled "radial hinge", is shown in this inset, but not in Fig. 30 (entire wing). This is an artifact. In Omma, as in Tetraphalerus, this area has a short bending zone, which is strongly bent in the folded position; however there is no break in the cuticle or crossing by a fold (see Definitions above). The structure called "apical hinge" appears to be a slight buckling in the secondary sclerotization just beyond the apical cell and should also be deleted.

Figs 32-35. Archostemata. Apical section of RA $3+4=$ R4.

Fig. 32. Micromalthus. The oblique basal section of RA $3+4$ is a cross-vein; the true base of RA $3+4$ is represented by the dotted line just above cross-vein $\mathrm{r} 1$.

Fig. 33. Tetraphalerus. The cross-vein at the apex of the wedge cell is $\mathrm{CuA} 3+4$. $\mathrm{CuA} 2$ is not changed.

Fig. 34. Priacma. $\mathrm{RA} 3+4=\mathrm{RA} 4$. $\mathrm{CuA} 2$ is not changed.

Fig. 39. Coelostoma. $\mathrm{CuA} 2$ is not changed.

Fig. 40. Rygmodus. The vein labelled $\mathrm{CuP}$ is merely a sclerotized area surrounding $\mathrm{AA} 3+4$; the true $\mathrm{CuP}$ (not labelled) is the oblique vein forming the base of the wedge cell.

Fig. 42. Syntelia. Delete CuP.

Fig. 54. Pseudomicrocara. $\mathrm{MP} 3=\mathrm{MP} 4+\mathrm{CuA} 1+2$, MP 4 ? $=$ $\mathrm{CuA} 1+2$

Fig. 55. Macrohelodes. MP $3=\mathrm{MP} 4+\mathrm{CuA} 1+2, \mathrm{MP} 4$ ? $=$ $\mathrm{CuA} 1+2$. The fold passing through MP1+2 is an artifact of the wing preparation.

Fig. 56. Eucinetus. $\mathrm{MP} 3=\mathrm{MP} 4+\mathrm{CuA} 1+2, \mathrm{MP} 4=\mathrm{CuA} 2$. The extra division in the wedge cell $\left(=2^{\text {nd }}\right.$ cubito-anal cell $)$ is anomalous and was not found in subsequent wing dissections.

Fig. 60. Notodascillus. MP4 $=\mathrm{MP} 4+\mathrm{CuA} 1, \mathrm{CuA} 1=\mathrm{CuA} 2$.

Fig. 61. Artematopus. $\mathrm{MP} 3=\mathrm{MP} 4+\mathrm{CuA} 1, \mathrm{MP} 4=\mathrm{CuA} 2$.

Fig. 62. Schizopus. MP $3 \mathrm{a}=\mathrm{MP} 3, \mathrm{MP} 3 \mathrm{~b}=\mathrm{MP} 4+\mathrm{CuA} 1$, MP4a could not be found on subsequent wing dissections and must be an individual anomaly, MP4b $=\mathrm{CuA} 2$.

Fig. 63. Hemiopsida. $\mathrm{MP} 3 \mathrm{a}=\mathrm{MP} 3, \mathrm{MP} 3 \mathrm{~b}=\mathrm{MP} 4+\mathrm{CuA} 1$, $\mathrm{MP} 4=\mathrm{CuA} 2$.

Fig. 64. Pterotus. $\mathrm{CuA} 2$ is not changed. The forking of MP4+ $\mathrm{CuA} 1$, with the two end branches labelled as MP4 and $\mathrm{CuA1}$, is apparently an anomaly occurring on one side only. The opposite wing lacked this fork but had a similar fork on $\mathrm{CuA}$. This type of instability in the venation of the medial area is not uncommon among certain groups of Elateroidea (sensu lato). Within the elaterid subfamily Cebrioninae, a similar fork has been seen in Aplastus and Wallace \& Fox (1980) reported forks in both of these veins within a series of specimens of another cebrionine, Scaptolenus lecontei (Sallé). All of these taxa have soft-bodies, short-lived males and brachelytrous or neotenic females; however it is likely that individual wing anomalies extend beyond this particular group.

Fig. 65. Pseudotetralobus. $\mathrm{CuA} 2$ is not changed.

Fig. 67. Lepidopteryx. MP $3=\mathrm{MP} 4+\mathrm{CuA} 1, \mathrm{MP} 4=\mathrm{CuA} 2$.

Fig. 68. Archetypus. $\mathrm{CuA} 2$ is unchanged.

Fig. 91. Artematopus wing folding diagram. The radial hinge should be removed. Although there was a slight buckling in one of the wings, other specimens have a continuous bending zone here.

Received August 19, 2002; revised October 9, 2003; accepted November 5, 2003 